\title{
On neural integration of faces and semantics
}

Citation for published version (APA):

van den Hurk, J. (2013). On neural integration of faces and semantics. [Doctoral Thesis, Maastricht University]. Maastricht University. https://doi.org/10.26481/dis.20130419jh

Document status and date:

Published: 01/01/2013

DOI:

10.26481/dis.20130419jh

Document Version:

Publisher's PDF, also known as Version of record

\section{Please check the document version of this publication:}

- A submitted manuscript is the version of the article upon submission and before peer-review. There can be important differences between the submitted version and the official published version of record.

People interested in the research are advised to contact the author for the final version of the publication, or visit the DOI to the publisher's website.

- The final author version and the galley proof are versions of the publication after peer review.

- The final published version features the final layout of the paper including the volume, issue and page numbers.

Link to publication

\footnotetext{
General rights rights.

- You may freely distribute the URL identifying the publication in the public portal. please follow below link for the End User Agreement:

www.umlib.nl/taverne-license

Take down policy

If you believe that this document breaches copyright please contact us at:

repository@maastrichtuniversity.nl

providing details and we will investigate your claim.
}

Copyright and moral rights for the publications made accessible in the public portal are retained by the authors and/or other copyright owners and it is a condition of accessing publications that users recognise and abide by the legal requirements associated with these

- Users may download and print one copy of any publication from the public portal for the purpose of private study or research.

- You may not further distribute the material or use it for any profit-making activity or commercial gain

If the publication is distributed under the terms of Article $25 \mathrm{fa}$ of the Dutch Copyright Act, indicated by the "Taverne" license above, 
On neural integration of faces and semantics 
(c) J. van den Hurk, 2013

Cover: J. van den Hurk

Chapter art: J.S. Mrofcynski

Production: CPI Wöhrmann Print Service B.V.

ISBN: 978-94-6203-339-9

The work presented in this thesis was funded by the Netherlands Organization for Scientific Research (NWO) and was conducted at Maastricht University. 


\title{
On neural integration of faces and semantics
}

\author{
Dissertation
}

To obtain the degree of Doctor at Maastricht University, on the authority of Rector Magnificus, Prof. dr. L.L.G. Soete, in accordance with the decision of the Board of Deans, to be defended in public on Friday $19^{\text {th }}$ of April 2013 at 10.00 hours

by

Job van den Hurk 


\section{Promotor}

Prof. dr. B. Jansma

\section{Co-promotor}

Prof. dr. E. Formisano

\section{Assessment Committee}

Prof. dr. B. de Gelder (Chair)

Prof. dr. H. Op de Beeck (KU Leuven, Belgium)

Prof. dr. A. Sack

Dr. G. Valente

Dr. V. Goffaux (KU Leuven, Belgium) 


\section{Content}

Introduction ....................................................................... 7

1.1 Faces in the brain .............................................................. 8

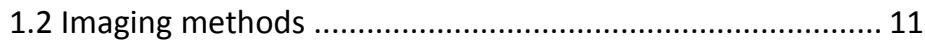

1.3 Statistical methods............................................................ 16

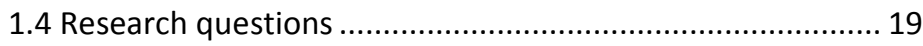

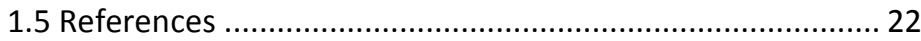

What's Behind a Face: Person Context Coding in Fusiform Face Area as Revealed by Multi-Voxel Pattern Analysis.....25

2.1 Introduction ..................................................................... 27

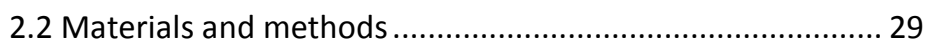

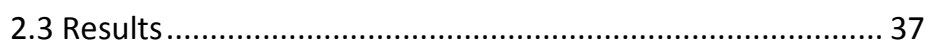

2.4 Discussion..................................................................... 41

2.5 Conclusion ....................................................................... 45

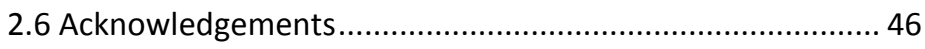

2.7 References ................................................................. 47

FFA - Precuneus Interaction During Processing of Words in Biographic Context ..........................................................53

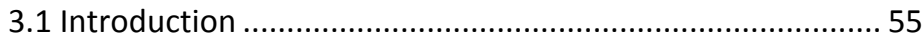

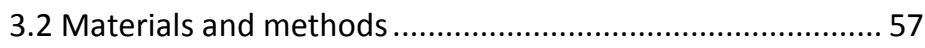

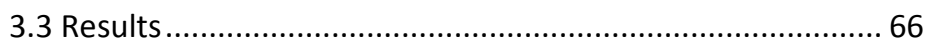

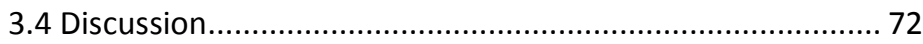

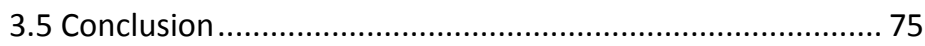

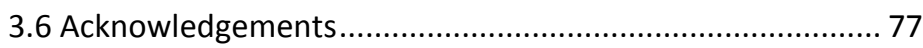

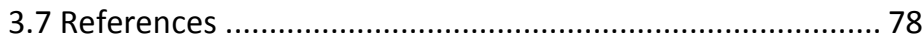

Functional Segregation of Mid- and Posterior 'Fusiform Face

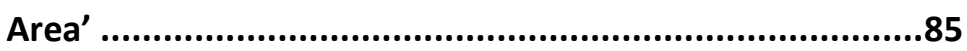

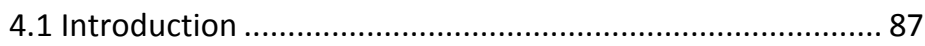

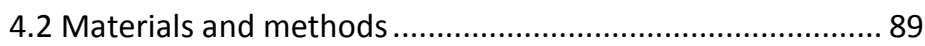

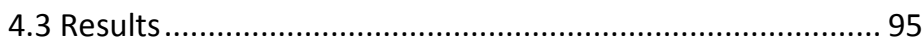

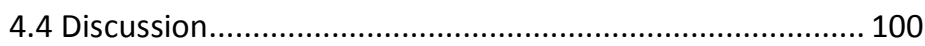

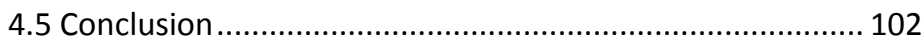

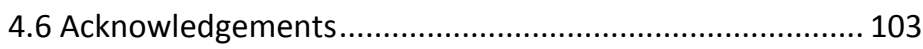

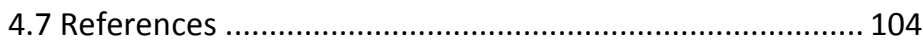


Structural Pathway Connects Anterior Inferior Temporal Cortex With Precuneus and Hippocampus: a DTI

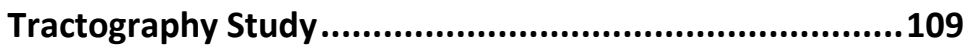

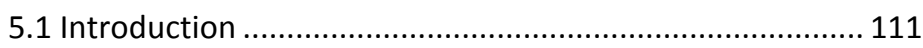

5.2 Materials and methods ................................................... 113

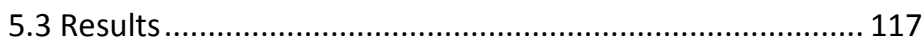

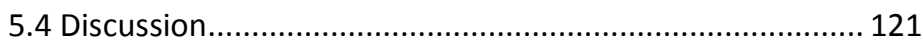

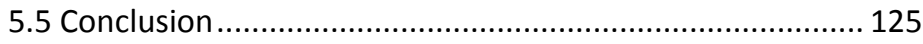

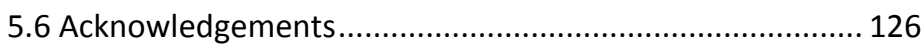

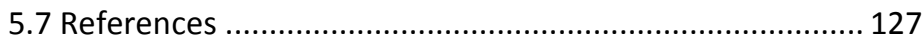

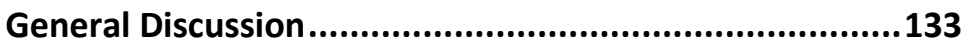

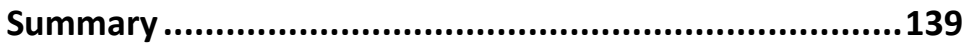

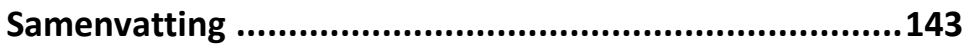

Acknowledgements..........................................................147

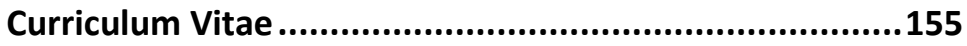




\section{Chapter 1}

Introduction 


\subsection{Faces in the brain}

\subsubsection{Meeting someone new}

Imagine sitting in a stand-up comedian café where an unknown person is about to do an act. While watching his performance, you can't help to think that you have seen his face before. Your brain starts digging when suddenly it hits you: this guy goes to the same supermarket as you do. Every Saturday morning during the weekly groceries you see him wandering through the aisles deciding upon his dinner for that night. After this evening in the comedy café, every time you walk through the supermarket on Saturday morning you realize that you see that same face weighing fruits or standing in the queue: the face has become familiar to you. Somehow, this face identity is stored in our brain, where it is used as some sort of 'template' against which perceived faces are matched to see if they are familiar (Bruce \& Young, 1986). Now compare this situation to the following. A new colleague is introduced at work. Her face is obviously unfamiliar at the time your boss asks her to give a short introduction. She tells her name, that she is an avid piano player and that she is happily married to her boyfriend who she met in high school. She does not like pasta, reads a lot of comic books and occasionally visits rock festivals. The next time your boss tells you about your new colleague, about her love for piano music and festivals, you immediately recall her face. Apparently, the biographical information she gave during her introduction is stored in your brain. Moreover, this information is linked to the stored representation of her face and is accessible as soon as you perceive her face. Vice versa, as soon as someone describes specific semantic features about her identity the face is retrieved from your memory (Craigie \& Hanley, 1993, 1997).

Both the situations that are described above illustrate an example of getting to know someone new. Still, there is an important difference between the two cases: in the first, the identification of the person's faces is based merely on visual features. The face was recognized as being familiar, because the unique constellation of the facial features such as eyes, nose and mouth was stored in your brain before. In the second situation, the identity of your new colleague was expanded with a semantic dimension. The information that was provided seems to have constructed a conceptual identity that is represented independently from the visual identity. Still, these two identity dimensions are highly interrelated, as hearing a brief description about a person is often enough to recall a visual image of the related face.

\subsubsection{Neural correlates of face perception}

From an evolutionary perspective, the skill to recognize the face of other members of the same species is of vital importance for survival (Leopold \& Rhodes, 2010). It may 
therefore come as no surprise that through the course of evolution, the human brain has developed a cortical region with a functional dedication to face processing (Kanwisher, 2000; Kanwisher, McDermott, \& Chun, 1997; Puce, Allison, Gore, \& McCarthy, 1995). Found on the ventro-temporally located fusiform gyrus, FFA shows a particular strong response to face stimuli in comparison to objects or houses. Already decades ago, the neuropsychological syndrome prosopagnosia indicated the likelihood of such a distinct cortical machinery specialized for face perception (Hadjikhani \& de Gelder, 2002; Kanwisher \& Yovel, 2006). Prosopagnosia patients commonly suffer from the disability to recognize faces but still have an intact concept of a person. This suggests that visual and conceptual identity are represented in a different manner in the brain.

The FFA lies on what is commonly referred to as the ventral visual pathway, also known as the 'what'-pathway. This pathway runs from early visual cortex in the occipital lobe, via the ventral surface of the temporal lobe towards anterior inferior temporal cortex. In contrast to the dorsal, or 'where/how' path, the ventral pathway is known to be involved in the identification of visual objects, as well as the representation of a few (discrete) category boundaries such as faces, places, limbs and tools. Several electrophysiology- and neuroimaging studies have demonstrated that the global organization of this ventral temporal area runs from a coarse object identification near the posterior regions, to a more specialized and complex representation towards anterior structures. This gradual increase in specialization along the posterior-anterior axis gives us a global idea about the level of processing a particular region on this part of the brain is involved in. When we look at the processing of faces, for instance, there are at least three local regions identifiable in this part of the brain that are responsive to face stimuli. The early visual face cluster is located on the junction of the occipital and temporal lobe and can be considered as the most posterior region sensitive to faces. Then, on the inferior occipital gyrus, Occipital Face Area or OFA can be found. Most anterior of these regions is FFA, and is therefore considered to be the region that is most 'specialized' in processing faces.

\subsubsection{Person identity: visual and non-visual features}

What exactly comprises the identity of a face? As described in section 1.1.1, there is more to face identity than mere visual features: biographic and semantic knowledge that is acquired over time creates a new dimension of identity. To what extent is FFA sensitive to either dimensions? The knowledge base that has developed over the years largely focussed on the visual aspect of identity. Numerous studies suggested that FFA is sensitive to the visual identity of a face (Andrews \& Ewbank, 2004; Barton, Press, Keenan, \& O'Connor, 2002; Gauthier, et al., 2000; Grill-Spector, Knouf, \& Kanwisher, 2004; Haxby, Hoffman, \& Gobbini, 2002; Hoffman \& Haxby, 2000; Loffler, Yourganov, Wilkinson, \& Wilson, 2005), but that the actual representation of identity is maintained in the anterior inferotemporal cortex or aIT (Brambati, Benoit, Monetta, 
Belleville, \& Joubert, 2010; Evans, Heggs, Antoun, \& Hodges, 1995; Gainotti, Barbier, \& Marra, 2003; Kriegeskorte, Formisano, Sorger, \& Goebel, 2007; Sergent, Ohta, \& MacDonald, 1992; Tranel, 2006; Tranel, Damasio, \& Damasio, 1997). From there, information might be fed back to FFA when this information is required for further processing (Kriegeskorte, et al., 2007).

The study of the non-visual dimension of identity has received much less attention. An interesting study by von Kriegstein and colleagues demonstrated that activity in FFA is modulated when subjects are engaged in a voice identification task. When participants heard a familiar voice, FFA showed a stronger response when the participants were asked to identify that voice than when they were performing a syllable counting control task (von Kriegstein, Kleinschmidt, Sterzer, \& Giraud, 2005). In addition, direct structural connections between voice regions (located on the planum temporale) and FFA have been revealed by diffusion MRI measures (Blank, Anwander, \& von Kriegstein, 2011). These findings suggest that, to some extent, FFA has access to information about an identity independently of visual information. Could this also be the case for more complex identity features that lie beyond the sensory modalities, such as semantic information? The lack of attention that this domain has received over the years, leaves numerous important questions about the extent of interaction between visual and conceptual identity unanswered. Before we focus more on the nature of these questions and the strategies that are required to address them, a brief introduction in the imaging methods as well as the analysis methods used in cognitive neuroscience is of significant merit. 


\subsection{Imaging methods}

In cognitive neuroscience, a wide variety of methods is employed. The experiments described in this thesis make use of two of these methods, both based on principles of Magnetic Resonance Imaging (MRI): functional MRI and a variant of diffusion MRI. These techniques are briefly discussed below.

\subsubsection{Functional Magnetic Resonance Imaging (fMRI)}

Functional Magnetic Resonance Imaging or fMRI is an imaging technique that can be used to indirectly measure brain activity. Its procedure is largely similar to that of standard MRI, which shall be briefly discussed. An MRI-scanner makes use of the physiological fact that body (and also brain) tissue contains a lot of water. Water molecules, in turn, are composed of 2 hydrogen atoms that are covalently bonded to one single oxygen atom. Hydrogen, or $\mathrm{H}$, consists of one single proton and is therefore the lightest chemical element. When a person is placed inside the strong magnetic field of the MRI-scanner, the protons in his or her body align with the direction of the magnetic field. In this aligned state, the rotational axis of the protons produce a high frequency rotation movement along the direction of the magnetic field, a process called 'precession'. The frequency at which this precession takes place depends on the strength of the magnetic field, and can be computed with the Larmor-equation: $\omega=\gamma B$, where $\omega$ is the precession frequency, $\gamma$ is the gyromagnetic ratio of a given particle and $B$ is the field strength of the external magnetic field. At a typical field strength of 3 Tesla, the precession frequency of hydrogen is $127,7 \mathrm{MHz}$. The radio-frequency coil, or RFcoil, then emits a radio-frequency pulse at the precession frequency of the protons, causing the protons to briefly alter their magnetization alignment. As soon as the RFpulse is switched off, the protons return to their original state. Two types of this socalled relaxation are commonly distinguished: longitudinal $\left(\mathrm{T}_{1}\right)$ relaxation, based on the protons returning to their original energy state, and transverse $\left(\mathrm{T}_{2}\right)$ relaxation, based on loss of phase coherence of the protons in precession. These different relaxation types entail different uses: $T_{1}$ relaxation speed depends on tissue type and is therefore suitable for imaging anatomy at high resolution (see figure 1.1A). $\mathrm{T}_{2}$ relaxation is induced by small inhomogeneities in the magnetic field, leading to variable Larmor frequencies across tissue.

Functional MRI is based on a special variant of a $\mathrm{T}_{2}$ weighted contrast: the $\mathrm{T}_{2}{ }^{*}$ (see figure 1.1B). When a group of neurons is activated by a particular task, they start consuming glucose and oxygen. An excessive amount of red blood cells (containing hemoglobin) carrying oxygen is transported to the neurons that are demanding energy. The overload of oxygenated hemoglobin $\left(\mathrm{Hb}-\mathrm{O}_{2}\right)$ at the active location is the key to fMRI: $\mathrm{Hb}-\mathrm{O}_{2}$ is diamagnetic (virtually nonmagnetic), whereas 
deoxygenated hemoglobin $(\mathrm{dHb})$ is paramagnetic. Since the $\mathrm{dHb}$ slightly alters the local field strength, this leads to a variable Larmor frequency hence to a faster transverse relaxation. When an abundance of $\mathrm{Hb}-\mathrm{O}_{2}$ is present, less distortion of the magnetic resonance signal is measured. It is the contrast between $\mathrm{Hb}-\mathrm{O}_{2}$ and $\mathrm{dHb}$ that is of interest in neuroimaging research. It should be clear that fMRI does not directly measure neural activity. Instead, it measures blood flow to and from regions that were active during performance. The signal that is measured is called the Blood-Oxygen Level Dependent signal, or BOLD-signal. It has been found that the BOLD-signal is not directly related to neural firing activity, but rather to the local field potential (LPF) of neurons (Logothetis, Pauls, Augath, Trinath, \& Oeltermann, 2001).

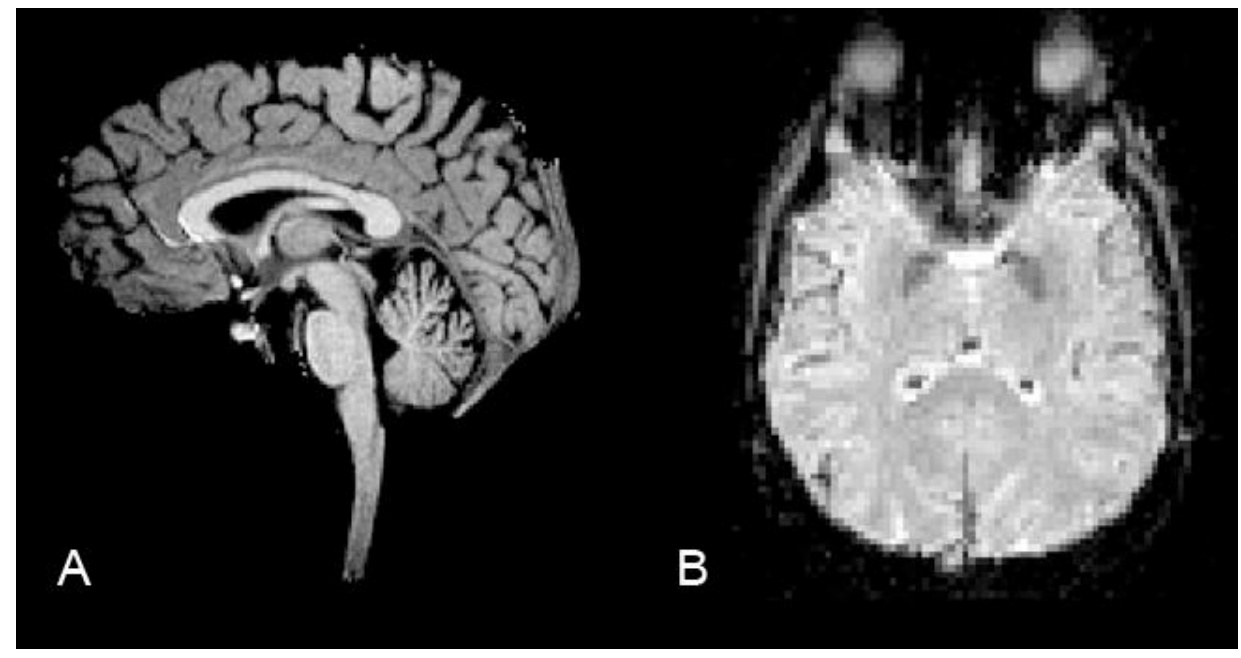

Figure 1.1. a. $T_{1}$ weighted anatomical image of the brain (sagittal cross section) $b . T_{2}{ }^{*}$ weighted functional image of the brain (transversal cross section).

Depending on various factors such as the magnetic fields strength and the particular imaging sequence used, the resolution of the MRI images can differ. On a typical 3 Tesla scanner, anatomical images can be obtained at a millimeter resolution or even below. Functional images can be acquired at approximately 3 millimeter accurately. In contrast to a 'flat' picture taken with a photo camera, an MRI scanner measures a 3D volume. Therefore, the resolution is not defined in pixels, but in 'volume pixels', or voxels. At higher field strengths (7 Tesla or even 9,4 Tesla), the voxel size can be reduced, with functional voxels reaching sub-millimeter levels. This spatial accuracy of the measurement is also referred to as the spatial resolution of an imaging method. For current standards, fMRI has a relatively high spatial resolution compared to other methods such as EEG, MEG and PET. 
In contrast to the high spatial resolution of fMRI, the accuracy of the measurements in time, the so-called temporal resolution, is relatively poor. Due to the fact that fMRI does not directly measure neural activity but rather hemodynamics, a typical lag of around 6 seconds can be observed between the actual occurrence of a neural event and the BOLD-signal that can be measured. This low temporal resolution creates a challenge for research questions with a temporal aspect, such as a causal relation between two neural events, even though there are advanced acquisition protocols that result in a temporal resolution of around $100 \mathrm{~ms}$ (Formisano, et al., 2002).

When a person is participating in an fMRI-study, he or she is often actively engaged in a task that typically involves a cognitive process like perception, mental imagery, working memory or decision making. During the performance of the task, the scanner needs to keep constant track of varying brain activity over time. Therefore, in predefined intervals, the scanner measures the BOLD-signal in all voxels. This interval between two measurement is also referred to as the repetition time, abbreviated as TR. In a typical experiment, the TR is around 2 seconds, but this can vary depending on the goal of the experiment. If, for instance, an experimental run lasts for 10 minutes, (10* $60 \mathrm{~s}) / 2 \mathrm{~s}=300$ volumes are acquired. These $3 \mathrm{D}$ volumes can be concatenated into a 4D dataset with time as $4^{\text {th }}$ dimension. For each voxel in a certain brain region, the BOLDsignal changes over time can be inspected and, after appropriate preprocessing steps, statistically analyzed.

\subsubsection{Diffusion Tensor Imaging (DTI)}

Diffusion Tensor Imaging or DTI is a special MRI technique that allows for accurately mapping the directions of the white matter connections in the brain. Its fundaments are based on the diffusivity of water molecules. Normally, the micromovements of water molecules are free: the molecules can move in all spatial directions (isotropic diffusion). However, when restricted by, for instance, cell membranes or fibers, the molecules can only move in a given direction (anisotropic diffusion). As this is the case in nerve fibers, this property can be employed to extract information about the directions of the diffusion in these fibers.

By using a specific magnetic field gradient pulse sequence (Stejskal \& Tanner, 1964), the diffusion direction is encoded in the MRI signal (Le Bihan, et al., 2001). In the resulting images, only those molecular displacements that occur along the gradient direction are visible. By changing the direction of this gradient pulse, differences in voxel signal reflect the diffusion anisotropy in the underlying tissue. During the subsequent analysis of the images, the direction within each voxel is quantified. This direction is typically expressed as a 3D diffusion tensor, which can be interpreted as an ellipsoid. If the diffusion of the water molecules within a voxel is equal in all directions and hence isotropic, the shape of this diffusion tensor will be close to a sphere. When 
the diffusivity is anisotropic, however, the tensor will be ellipsoid. Where this tensor quantifies the direction of diffusion in a voxel, it provides no information on the strength of the diffusion. To estimate this, the fractional anisotropy (FA) parameter can be computed. This FA value represents the mathematical comparison of the three eigenvalues of the three eigenvectors (representing the directions) of the tensor. An FA value close to 0 represents a near-isotropic diffusion, whereas an FA value approximating 1 represents strong anisotropy (see figure 1.2).

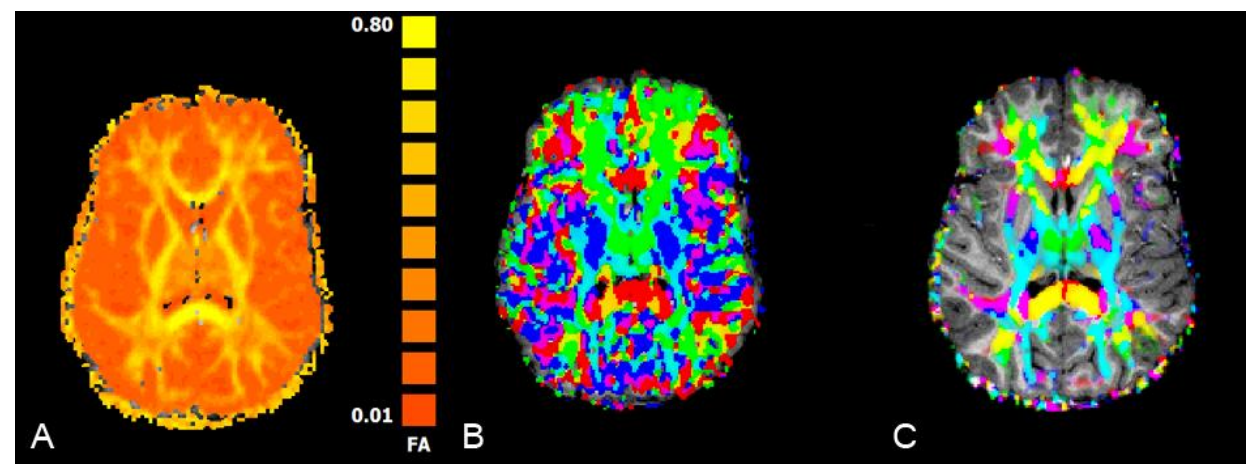

Figure 1.2. a. Fractional anisotropy (FA) map (yellow $=$ high FA magnitude). $b$. Diffusion tensor direction map. Different directions are color coded (left-right: red, ventral-dorsal: blue, anteriorposterior: green.) c. Diffusion tensor direction map weighted with the underlying FA values. As can be seen in a., the FA value is highest in the white matter where the fiber bundles generally have a strong directionality. The tensor directions shown in $b$. give a coarse impression of the diffusion directions across the presented transversal slice. However, this view provides no information on the strength of the diffusion. Therefore, in c., the tensors are weighted with the FA value that is computed in the corresponding voxels. This allows for a more robust interpretation, as this map combines the magnitude of anisotropy with the direction of the tensors. The result is a elaborated systems of fiber tracks within the white matter bundles, coded in separate colors per bundle.

The computed tensors allow for an informative tracking of particular fibers. During this fiber tracking analysis, one or more given regions of interest are used as seed region. From this seed region, the tensors of neighboring voxels are connected into a fiber in a way that best follows the diffusion direction of those voxels. In addition, this analysis allows for tracing specific fibers that connect two regions.

In summary, DTI and related analysis methods provide an interesting opportunity to characterize structural connections between brain regions. Still, with a typical voxel size of $\pm 2 \times 2 \times 2 \mathrm{~mm}$, diffusion tensor imaging samples the diffusion across thousands of fibers and thus represents a noisy average of the sampled voxel 
volume. Therefore, one has to interpret the results with some caution, especially whenever they are used to speculate about their role in cognitive information transfer in functional networks. 


\subsection{Statistical methods}

Of all the available statistical approaches that are used in the analysis of neuroimaging data, two general classes of approaches will be discussed in this section: univariate and multivariate analysis. Both have a lot of applications in basically all scientific domains, and both have their own practicalities. In this section, the general difference between univariate and multivariate analysis of imaging data will be discussed, as both methods are commonly referred to throughout this thesis.

\subsubsection{General Linear Model (GLM)}

For the analysis of neuroimaging data, the General Linear Model (GLM) is a very powerful method and is often used when two or more experimental conditions are to be compared. It is used to 'explain' the variance within the time course (BOLD signal change) within a voxel across conditions. As input for the GLM, one usually takes the signal from a given time interval based on information on the on- and offset of particular experimental conditions during data acquisition, defined as predictors in the model. This information is translated into a so-called design matrix $(\mathrm{X})$. This matrix contains the predictors and corresponding expected time course per condition. During the subsequent GLM fitting procedure (based on multiple regression), the GLM algorithm searches for an optimal way to explain the variance in a voxel's time course in terms of a linear combination of the predictors in the design matrix. For each predictor in the matrix, a beta value (b) is obtained that quantifies how well the corresponding condition fits with the actual time course of a voxel. The official form of the GLM is $y=X b+e$, where $\mathrm{y}$ represents the actual voxel data, $\mathrm{X}$ is the design matrix, $b$ is the vector of resulting beta's and e is error. As can be implied from this, after fitting the model to the actual data, the matrix-vector multiplication $\mathrm{Xb}$ plus the error term e gives an estimation of the true data $y$.

The GLM is very suitable when one is interested in comparing the responses of two or more conditions. In addition, these results are generally well interpretable in terms of directionality and location (an observed high explained variance results in assuming high correlated neural activity for a given condition in a given region), as well as the relative contribution of individual predictors to the measured signal (one condition results in higher neural activation than another).

\subsubsection{Multi-Voxel Pattern Analysis (MVPA)}

The concept of Multi-Voxel Pattern Analysis (also abbreviated as MVPA) is not a specific analysis type itself, but rather incorporates several distinct approaches. Whereas a univariate method such as a GLM compares the absolute difference between BOLD- 
responses elicited by two or more conditions within a region, MVPA considers the difference between distributed patterns of activity (see figure 1.3).
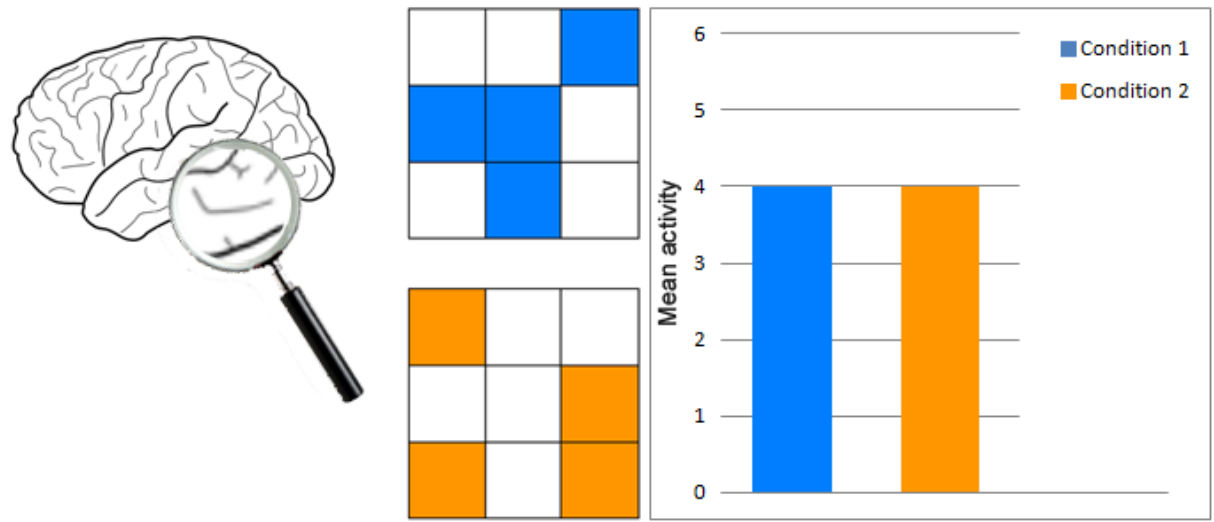

Figure 1.3. Response patterns to condition 1 and 2 within a region comprising 9 voxels. As can be seen, both conditions elicit completely distinct patterns of activity (left, MVPA detects this difference), while the region's average response to both conditions is similar (right, univariate GLM would not detect any difference). This example illustrates the gain in sensitivity that MVPA has over univariate statistics.

For each condition that is to be analyzed, response patterns are created by estimating each voxel's response to a certain experimental condition. During a subsequent training phase, a computer algorithm 'learns' the relationship between a set of patterns and the corresponding condition labels. After training, a test set of patterns that have not been used during the training phase are presented to the trained algorithm which classifies them as belonging to one of the condition classes. The classification performance is quantified as prediction accuracy. A core feature of MVPA is how to interpret the resulting accuracy values of this prediction procedure. MVPA assumes that the accuracy is an indication of the presence of information: if the accuracy lies significantly above chance level, these patterns of activity somehow carry information about the neural information process in a given experimental condition.

Because of this procedure of decoding information from patterns of brain activity, MVPA methods are also often referred to as brain-reading or classification techniques. The validity of this method can be found in its attempt to respect the distributed nature of information encoding in the brain. In addition, it is known to be more sensitive to subtle differences in conditions than a GLM. The outcome of MPVA 
methods, however, are generally harder to interpret, especially when classification methods are used that are based on non-linear (radial or polynomial) kernel functions. 


\subsection{Research questions}

This thesis investigates the potential involvement of the Fusiform Face Area in the processing of semantic identity information, and possible functional and structural mechanisms that underlie this processing. Below, the precise research questions that are investigated in this thesis are briefly outlined.

\section{Does FFA have access to semantic information about a person?}

Previous work in the field of face identification mainly focused on the visual aspects of identity. This thesis focuses on higher order semantic information related to identity, where semantics refer to the knowledge we have about the world, stored in our memory. Because of the higher order nature of semantics and the dependency between the visual and semantic aspects of identity, a careful setup of the experimental design was developed in Chapter 2. We used words to trigger two types of semantic processes. In one condition, which we named the category condition, these words were presented in a categorical context (cars, professions, sports etc.). In the other condition, the person condition, the same words were used but this time in the context of a curriculum vitae (in keywords) describing a person. We measured the response patterns in FFA using fMRI, and used MVPA to investigate whether these patterns differed between the two semantic contexts.

\section{Are other brain regions sensitive to the person/category context manipulation?}

In Chapter 2, the focus of analysis was solely on FFA. In Chapter 3, based on the findings in Chapter 2, we aimed to find out whether the observed sensitivity in FFA to the semantic context is a local phenomenon within FFA, or whether other brain areas are involved in processing semantic context. We extended our design and dataset to focus on the entire brain. We first performed a whole-brain GLM analysis to search for brain regions that possibly process the two conditions differently. Subsequently, we employed a searchlight technique to identify local clusters of voxels that show sensitivity towards the subtle context manipulation on a multivariate level.

Is there a functional connection between these brain regions that process the semantic contexts and FFA? If so, is this connection stronger during the processing of the person context than during the semantic context?

In Chapter 3, we also analyzed the functional connectivity between cortical clusters that were identified by the searchlight algorithm and FFA. By using a method related to psychophysiological interaction (PPI) analysis, the correlation between informative segments of the region's time courses was computed for the category and the person condition separately. We investigated whether the correlation coefficients between FFA 
and the cortical clusters were significantly stronger for the person condition than for the category condition.

Is there a functional segregation between the mid-and posterior fusiform cortex during processing of identity?

The technical advances of the recent years have led to an improvement of spatial resolution in functional imaging. Recently, this in turn led to an anatomical reconsideration of FFA. By paying close attention to anatomical landmarks and neighboring functional modules, at least two clusters can be identified that were previously combined into what is now known as FFA. These clusters, located on the middle fusiform sulcus (mid fusiform or mFus) and on the posterior fusiform gyrus (posterior fusiform or pFus) both show a strong response to faces compared to houses and scrambled faces. It is unknown, however, whether the exact role of these patches differs when it comes to processing the identity of a face. In Chapter 4, we investigated this issue by repeatedly presenting participants with 3 identities: first a face was presented, and after some time, 5 words followed that described a person in keywords. By using MVPA, we investigated whether the information content in the mid- and posterior fusiform clusters differed during the processing of the face and the semantics.

Does disentangling FFA into a mid-and a posterior fusiform face region result in a gain in the information that can be decoded from these regions?

Previous studies in the field have investigated whether information about face identity was salient enough in FFA to be decoded from patterns of activity, without clear results. In Chapter 4, we tested whether making a distinction between mFus and pFus increases the accuracy at which identity information can be retrieved from activity patterns, by comparing the classification results from segregated FFA clusters with 'traditional' FFA (i.e. not subdivided).

How are regions that are known to play a role in identity processing structurally connected to each other?

When associating semantic information about a person with a face, several regions have been found to be functionally involved. Fusiform face regions play a role in the processing of the face information, thereby presumably receiving important information from anterior inferior temporal cortex (aIT). In Chapter 3 we demonstrated that the precuneus connects with fusiform face regions during processing of the semantic identity information, presumably playing a role in the association process. The identity construct that is the result of the association of face and corresponding background knowledge is likely memorized via the hippocampus. How are these 4 regions (fusiform regions, precuneus, aIT and hippocampus) structurally connected to each other? In 
Chapter 5, we used diffusion tensor imaging (DTI) and region-of-interest (ROI) based fiber tracking to identify the white matter tracts that likely form the face-identityassociation network. 


\subsection{References}

Andrews, T. J., \& Ewbank, M. P. (2004). Distinct representations for facial identity and changeable aspects of faces in the human temporal lobe. Neuroimage, 23(3), 905-913.

Barton, J. J., Press, D. Z., Keenan, J. P., \& O'Connor, M. (2002). Lesions of the fusiform face area impair perception of facial configuration in prosopagnosia. Neurology, 58(1), 71-78.

Blank, H., Anwander, A., \& von Kriegstein, K. (2011). Direct structural connections between voice- and face-recognition areas. J Neurosci, 31(36), 12906-12915.

Brambati, S. M., Benoit, S., Monetta, L., Belleville, S., \& Joubert, S. (2010). The role of the left anterior temporal lobe in the semantic processing of famous faces. Neuroimage, 53(2), 674-681.

Bruce, V., \& Young, A. (1986). Understanding face recognition. Br J Psychol, 77 ( Pt 3), 305-327.

Craigie, M., \& Hanley, J. R. (1993). Access to visual information from a name is contingent on access to identity-specific semantic information. Memory, 1(4), 367-391.

Craigie, M., \& Hanley, J. R. (1997). Putting faces to names. Br J Psychol, 88 ( Pt 1), 157-171.

Evans, J. J., Heggs, A. J., Antoun, N., \& Hodges, J. R. (1995). Progressive prosopagnosia associated with selective right temporal lobe atrophy. A new syndrome? Brain, 118 ( Pt 1), 1-13.

Formisano, E., Linden, D. E., Di Salle, F., Trojano, L., Esposito, F., Sack, A. T., et al. (2002). Tracking the mind's image in the brain I: time-resolved fMRI during visuospatial mental imagery. Neuron, 35(1), 185-194.

Gainotti, G., Barbier, A., \& Marra, C. (2003). Slowly progressive defect in recognition of familiar people in a patient with right anterior temporal atrophy. Brain, 126(Pt 4), 792-803.

Gauthier, I., Tarr, M. J., Moylan, J., Skudlarski, P., Gore, J. C., \& Anderson, A. W. (2000). The fusiform "face area" is part of a network that processes faces at the individual level. J Cogn Neurosci, 12(3), 495-504. 
Grill-Spector, K., Knouf, N., \& Kanwisher, N. (2004). The fusiform face area subserves face perception, not generic within-category identification. Nat Neurosci, 7(5), $555-562$.

Hadjikhani, N., \& de Gelder, B. (2002). Neural basis of prosopagnosia: an fMRI study. Hum Brain Mapp, 16(3), 176-182.

Haxby, J. V., Hoffman, E. A., \& Gobbini, M. I. (2002). Human neural systems for face recognition and social communication. Biol Psychiatry, 51(1), 59-67.

Hoffman, E. A., \& Haxby, J. V. (2000). Distinct representations of eye gaze and identity in the distributed human neural system for face perception. Nat Neurosci, 3(1), $80-84$

Kanwisher, N. (2000). Domain specificity in face perception. Nat Neurosci, 3(8), 759763.

Kanwisher, N., McDermott, J., \& Chun, M. M. (1997). The fusiform face area: a module in human extrastriate cortex specialized for face perception. J Neurosci, 17(11), 4302-4311.

Kanwisher, N., \& Yovel, G. (2006). The fusiform face area: a cortical region specialized for the perception of faces. Philos Trans R Soc Lond B Biol Sci, 361(1476), 2109-2128.

Kriegeskorte, N., Formisano, E., Sorger, B., \& Goebel, R. (2007). Individual faces elicit distinct response patterns in human anterior temporal cortex. Proc Natl Acad Sci U S A, 104(51), 20600-20605.

Le Bihan, D., Mangin, J. F., Poupon, C., Clark, C. A., Pappata, S., Molko, N., et al. (2001). Diffusion tensor imaging: concepts and applications. J Magn Reson Imaging, 13(4), 534-546.

Leopold, D. A., \& Rhodes, G. (2010). A comparative view of face perception. J Comp Psychol, 124(3), 233-251.

Loffler, G., Yourganov, G., Wilkinson, F., \& Wilson, H. R. (2005). fMRI evidence for the neural representation of faces. Nat Neurosci, 8(10), 1386-1390.

Logothetis, N. K., Pauls, J., Augath, M., Trinath, T., \& Oeltermann, A. (2001). Neurophysiological investigation of the basis of the fMRI signal. Nature, 412(6843), 150-157. 
Puce, A., Allison, T., Gore, J. C., \& McCarthy, G. (1995). Face-sensitive regions in human extrastriate cortex studied by functional MRI. J Neurophysiol, 74(3), 1192-1199.

Sergent, J., Ohta, S., \& MacDonald, B. (1992). Functional neuroanatomy of face and object processing. A positron emission tomography study. Brain, $115 \mathrm{Pt}$ 1, 1536.

Stejskal, E. O., \& Tanner, J. E. (1964). Spin Diffusion Measurements: Spin Echoes in the Presence of a Time-Dependent Field Gradient. J. Chem. Phys., 42(1), 5.

Tranel, D. (2006). Impaired naming of unique landmarks is associated with left temporal polar damage. Neuropsychology, 20(1), 1-10.

Tranel, D., Damasio, H., \& Damasio, A. R. (1997). A neural basis for the retrieval of conceptual knowledge. Neuropsychologia, 35(10), 1319-1327.

von Kriegstein, K., Kleinschmidt, A., Sterzer, P., \& Giraud, A. L. (2005). Interaction of face and voice areas during speaker recognition. J Cogn Neurosci, 17(3), 367376. 


\section{Chapter 2}

\section{What's Behind a Face: Person Context Coding in Fusiform Face Area as Revealed by Multi-Voxel Pattern Analysis ${ }^{1}$}

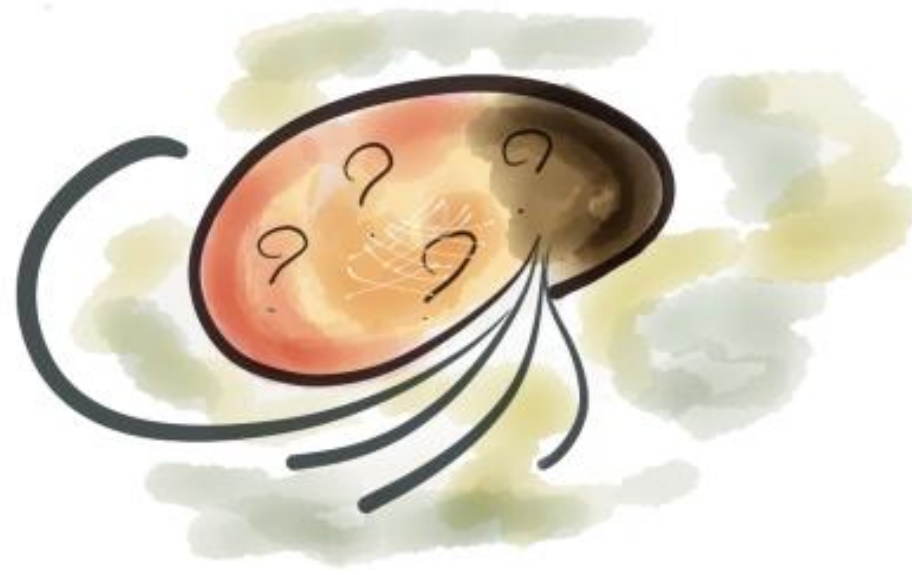

${ }^{1}$ Van den Hurk, J., Gentile, F., \& Jansma, B. M. (2011). What's Behind a Face: Person Context Coding in Fusiform Face Area as Revealed by Multi-Voxel Pattern Analysis. Cereb Cortex, 21(12), 2893-2899. 


\section{Abstract}

The identification of a face comprises processing of both visual features and conceptual knowledge. Studies showing that the Fusiform Face Area (FFA) is sensitive to face identity generally neglect this dissociation. The present study is the first that isolates conceptual face processing by using words presented in a person context instead of faces. The design consisted of two different conditions. In one condition, participants were presented with blocks of words related to each other at the categorical level (e.g. brand of cars, European cities). The second condition consisted of blocks of words linked to the personality features of a specific face. Both conditions were created from the same $8 * 8$ word matrix, thereby controlling for visual input across conditions. Univariate statistical contrasts did not yield any significant differences between the two conditions in FFA. However, a machine learning classification algorithm was able to successfully learn the functional relationship between the two contexts and their underlying response patterns in FFA, suggesting that these activation patterns can code for different semantic contexts. These results suggest that the level of processing in FFA goes beyond facial features. This has strong implications for the debate about the role of FFA in face identification. 


\subsection{Introduction}

When seeing the face of a friend, we first recognize it as being familiar. Instantly, we recall biographic information about him or her and retrieve his or her name. From a cognitive point of view, the identification of a face follows the matching of a set of facial features against hard-wired templates of known faces (Bruce \& Young, 1986). Once we recognize the perceived face as being someone familiar, we have instant access to person-specific semantic knowledge about that individual's occupation and socio-economic status (Bruce \& Young, 1986), followed by access to name (Craigie \& Hanley, 1993, 1997). The mechanism of face perception and the consequent identification is mediated by a complex network of brain regions (Haxby, Hoffman, \& Gobbini, 2000). But while the processing of facial stimuli is understood relatively well up to the level of face identification based on visual features, the stage of person-specific knowledge access remains unclear.

One specific brain region that has been shown play a central role in face processing is the Fusiform Face Area or FFA (Kanwisher, McDermott, \& Chun, 1997; Puce, Allison, Gore, \& McCarthy, 1995), a small region located on the ventral bank of the temporal lobe that shows a particular sensitivity towards faces over other stimuli, e.g. objects. Next to face recognition, a wealth of evidence showed that FFA is responsible for face identification (Andrews \& Ewbank, 2004; Barton, Press, Keenan, \& O'Connor, 2002; Gauthier, et al., 2000; Grill-Spector, Knouf, \& Kanwisher, 2004; Haxby, Hoffman, \& Gobbini, 2002; Hoffman \& Haxby, 2000; Loffler, Yourganov, Wilkinson, \& Wilson, 2005). However, several neuroimaging (Brambati, Benoit, Monetta, Belleville, \& Joubert, 2010; Kriegeskorte, Formisano, Sorger, \& Goebel, 2007; Sergent, Ohta, \& MacDonald, 1992) and lesion studies (Evans, Heggs, Antoun, \& Hodges, 1995; Gainotti, Barbier, \& Marra, 2003; Tranel, 2006; Tranel, Damasio, \& Damasio, 1997), suggested that actual facial identity is maintained in anterior inferotemporal cortex (aIT), and is then back-projected to FFA (Kriegeskorte, et al., 2007).

Although the identity of a familiar face comprises visual features and conceptual and biographic knowledge about that face, previous studies either did not dissociate those two components experimentally, or found no effects in FFA (Mur, Ruff, Bodurka, Bandettini, \& Kriegeskorte, 2010). Therefore, it remains still unknown whether the face identity representation in FFA is confined solely to visual features, or whether it is extended with coding for conceptual knowledge about a person. In order to address this issue, it would be valuable to 
investigate the role of FFA in the processing of semantic information about a face, in the absence of direct facial information (visual features).

In the present study, we hypothesize that FFA has access to a semantic context when no face is visible during the presentation of that context. Using high-field functional magnetic resonance imaging (fMRI), we measured the functional response of FFA in two different conditions: a person-specific (PER) and a categorical (CAT) condition. In the former, we presented blocks of words related to a specific face that was presented at the beginning of the block. The CAT condition consisted of a series of words all belonging to the same category. As the words used for both conditions (PER, CAT) were selected from the same set of stimuli, any item-related confound is to be excluded. We subsequently used a machine learning classifier (linear support vector machine) to analyze our data. This method allowed us to look at the neural response of FFA in a multivariate fashion. More specifically, instead of analyzing each and every voxel separately, we considered the pattern of activity of the total amount of voxels in FFA. We investigated whether the two different contexts (PER, CAT) elicited different patterns.

The use of pattern recognition as method to analyze fMRI data has proven to be an effective way of decoding brain states from distributed patterns of activity rather than focusing on the average response of an entire region (Cox \& Savoy, 2003; Formisano, De Martino, Bonte, \& Goebel, 2008; Haynes \& Rees, 2006; Kamitani \& Tong, 2005; Mur, Bandettini, \& Kriegeskorte, 2009; Norman, Polyn, Detre, \& Haxby, 2006; Pereira, Mitchell, \& Botvinick, 2009). 


\subsection{Materials and methods}

\subsubsection{Participants}

Seven right-handed healthy adults with normal or corrected to normal vision participated in this study (five females, mean age 24.3 years old). Participants were screened for fMRI compatibility, signed informed-consent and were financially compensated for their time. After acquisition, one participant had to be excluded from the analysis as it was not possible to localize FFA. The study was approved by the ethical committee of the Faculty.

\subsubsection{Stimuli}

We created two conditions from an $8 * 8$ word matrix (table 2.1), consisting of 8 categories (professions, capital cities, house types, sports, hobbies, domestic animals, music styles and car brands). The words used were all Dutch, the native language of all participants. For the category (CAT) condition, 8 blocks each consisting of 8 words were created by using the items from a single category per block (the columns in the word matrix). Each CAT block thus represented 1 of 8 categories (i.e. "professions"). For the person (PER) condition, 8 blocks each consisting of 8 words were created by using one word from each category (the rows in the word matrix): each resulting sequence of 8 words could be interpreted as a keyword description of an individual profile (i.e. a surgeon living in Amsterdam, playing soccer etc.). By using this approach, we were able to create two distinct semantic contexts while controlling for visual and semantic input of individual items across conditions.

For the person and category condition, we used 8 different face images (provided by Mark Steyvers http://psiexp.ss.uci.edu/research/software.htm) and 8 images of objects (softball glove, house, car, book, dog, construction worker, globe and saxophone) as instruction for the person and category conditions respectively. 
Car City Job Dwelling Sports Hobby Pet Music

Toyota Amsterdam Surgeon $\begin{aligned} & \text { Terraced } \\ & \text { house }\end{aligned}$ Soccer Cooking Dog Ska

Hyundai Brussels Baker Apartment* Hockey Painting Cat Rock

Peugeot Paris Teacher Penthouse Ice skating Fishing Rabbit Classical music

Opel London Composer Villa Athletics Gardening Parakeet Pop

Suzuki Berlin Engineer Studio* Tennis Traveling Chicken House

Mercedes Madrid Fitter $\quad \begin{aligned} & \text { Travel } \\ & \text { trailer }\end{aligned} \quad$ Badminton $\quad \begin{aligned} & \text { Puzzle } \\ & \text { solving }\end{aligned} \quad$ Hamster Jazz

Renault Lissabon Barber Apartment Rowing Reading Goldfish Soul building

Rover Stockholm Editor House on Cricket Walking Lizard Blues

Table 2.1. $8 * 8$ word matrix. The two conditions used in this experiment were derived from this stimulus matrix. Both the category and person condition consisted of 8 blocks of 8 words, where each block was created by taking all 8 words from a column (category condition, i.e. "professions") or by taking 1 word from each column (person condition, i.e. a surgeon living in Amsterdam, playing soccer etc.). As the original matrix used consisted of Dutch words, the matrix has been translated to English for this chapter. Some Dutch words describing different concepts could not be translated literally (marked with an asterisk). 


\subsubsection{Procedure}

The experiment consisted of three runs in a block design. Each run consisted of 8 blocks from each condition (CAT, PER), and started with a fixation cross lasting for 26 seconds. At the beginning of each block an instructional image (face or object) was presented for 6 seconds. The image was followed by a fixation cross that lasted for 10 seconds. This interval allowed the BOLD response elicited by the stimulus in the instruction phase to return back to baseline before the onset of the actual block. Next, a block of 8 words was presented, where each word lasted for $1500 \mathrm{~ms}$, followed by $500 \mathrm{~ms}$ of fixation (see figure 2.1). Participants were instructed to pay close attention to the block of words and to associate each of them with the image in the instruction (i.e. to associate the category sports with the image of a softball glove, within the category condition). The intention of this approach was to encourage the participants to actively create a semantic context out of the presented words they were facing. They were informed that after each run, all images were presented one by one. The task of the participants was to recall as many word as possible related to that category or person. This additional debriefing phase was included to encourage participant to attend to the block of words inside the scanner. 


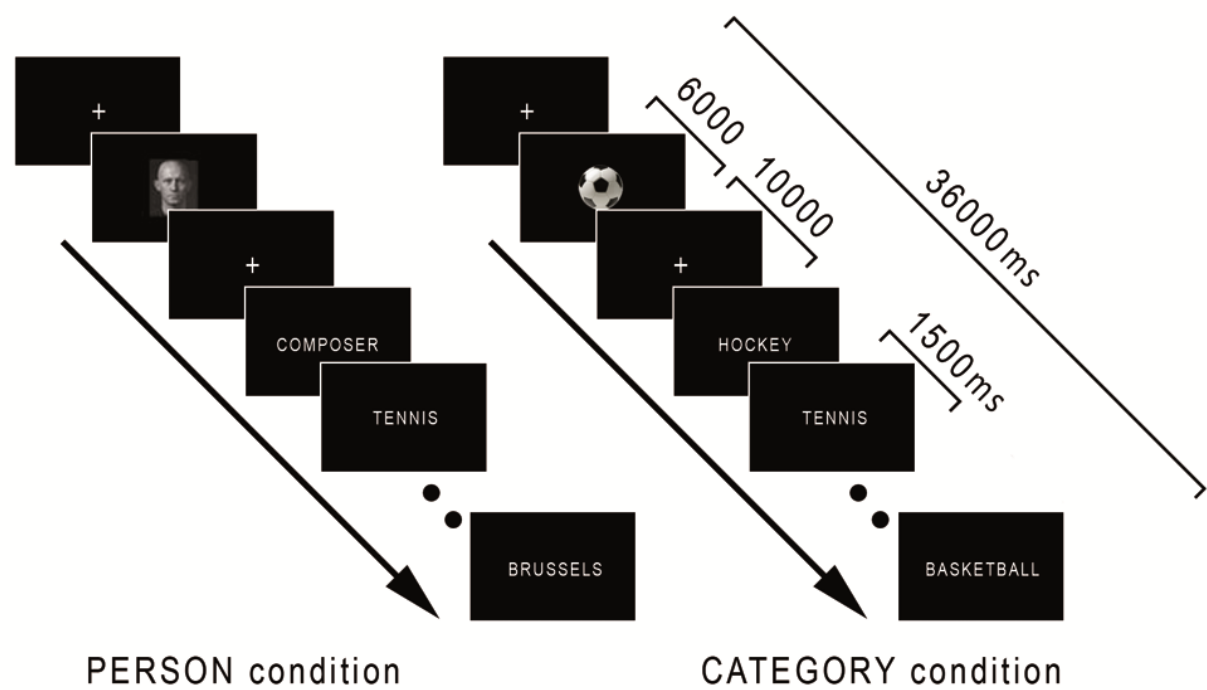

Figure 2.1. Experimental design. The figure illustrates one block of both conditions. A block started with a fixation period. follow by an instructional image of a face (left) or object (right) for 6 seconds. The image indicated which of the two contexts was about to be presented. The instruction "person" is given by a face. followed by a list of 8 words associated to the person's background (a composer who has tennis as hobby and lives in Brussels). The instruction "category" is given by an object (here a ball referring to category sports). followed by a list of 8 words from that category. The participant was instructed to associate the 8 words with the instructional image. To check if the participant was paying attention during the scanning, after each run, he or she was presented with all the instructional images and asked to recall as many keywords he or she remembered in combination with each image.

\subsubsection{Data acquisition}

Functional and anatomical images were acquired on a 3T Siemens Magnetom Allegra head scanner (Siemens Medical System, Erlangen, Germany) using a standard head coil. Thirty-two oblique axial slices $(3.5 \mathrm{~mm} \times 3.5 \mathrm{~mm}$, slice thickness $3,5 \mathrm{~mm}$, interslice distance $0 \mathrm{~mm}$ ) covering the entire cortical volume were collected using a standard echo planar-imaging (EPI) sequence (repetition time $[\mathrm{TR}]=2000 \mathrm{~ms}$, matrix size $=64 \mathrm{x} 64$, echo time $[\mathrm{TE}]=30 \mathrm{~ms}$ ). For each run, we collected 355 functional images, of which the first 2 were excluded due to $\mathrm{T} 1$ saturation. In addition to the experimental runs, an independent FFA 
localizer run was included. Anatomical images covering the whole brain were obtained after the experimental runs, using a $1 \times 1 \times 1 \mathrm{~mm}$ resolution T1weighted ADNI sequence $\left([\mathrm{TR}]=2250 \mathrm{~ms} ;[\mathrm{TE}]=2.6 \mathrm{~ms}\right.$; flip angle $\left.=9^{\circ}\right)$.

The participants were placed comfortably in the scanner and their head was fixed with soft foam pads. Participants saw the stimuli projected on a screen through a mirror mounted on the head coil. Stimulus presentation was synchronized with MR data acquisition by triggering the stimulus program (Presentation software, Neurobehavioral Systems Inc, Albany, CA) with the first MR pulse.

\subsubsection{Analysis}

Functional and anatomical data were pre-processed and analysed using the BrainVoyager QX 2.1 package (Brain Innovation, Maastricht, The Netherlands). Functional volumes were first corrected for slice scan-time differences and 3D head motion. In order to enhance the subsequent alignment of the functional images to the anatomical volume, the first and second runs were corrected with the third run as intra-session reference, as the acquisition of this run was temporally adjacent to the anatomical scan. Subsequently, linear trends and low frequency temporal drifts were removed from the data using a high-pass filter, removing temporal frequencies below 2 cycles per run. No spatial smoothing was performed. After the pre-processing, functional data were co-registered to the high-resolution anatomical volume and normalized to Talairach space.

For each participant, the location of the Fusiform Face Area was determined bilaterally by an independent localizer run, using grey scale images of faces, scrambled faces and houses. The procedure defines the FFA as the result of a conjunction analysis (FDR-corrected at $q=0.05$ ) between a face responsive contrast (\%BOLD signal change faces $>$ scrambled faces) and a face selective contrast (\%BOLD signal change faces $>$ houses).

Conventional univariate statistical analysis of functional data was performed on an average cortical surface, obtained by reconstructing both hemispheres for each participant. The surfaces were subsequently aligned using a cortex-based alignment procedure, an algorithm that uses individual curvature information to align corresponding gyri and sulci across participants (Goebel, Esposito, \& Formisano, 2006). The analysis was performed using a random effects GLM, using subject as random variable. A design matrix was created, using a predictor for both conditions. The predicted time courses were adjusted for the typical hemodynamic response delay by convolution with a canonical 
(double gamma) hemodynamic response function. Statistical contrasts were considered at the multi-subject whole-brain level, FDR corrected at q=0.05. We subsequently performed a region-of-interest (ROI) analysis of the person > category contrast on the individual subject level. We focused on individual left and right FFA as ROI, as identified by the independent localizer run.

The data were also analyzed via a pattern classification approach. We used a support vector machine (SVM), a model that performs binary classification on a dataset by placing all cases in a multidimensional space. Each individual case (or example) is expressed as a vector of $\mathrm{N}$ features in the $\mathrm{N}$ dimensional space. The examples are labelled as belonging to one of two experimental conditions. A SVM training algorithm then defines an N-1 dimensional hyperplane that optimally separates the data in two categories. The resulting model can be used to predict whether a new example falls into one category or the other, depending on what side of the hyperplane the example falls. Through this means, the accuracy of the trained model can be assessed by considering the ratio of correctly classified examples.

In the present study, the features were represented by the voxels identified by the FFA localizer. In addition, in order to analyze the right and left FFA independently, we considered two different feature-spaces comprising the voxels from the right and left FFA respectively. Each example in such a multidimensional space was represented by a multidimensional vector comprising the hemodynamic responses from all voxels in FFA (right or left) related to each of the 48 blocks measured along the 3 experimental runs. The a priori assumption on the region of interest decreases the amount of irrelevant features, thereby increasing the generalization performance of the classifier (Cox \& Savoy, 2003; Formisano, De Martino, \& Valente, 2008; Kamitani \& Tong, 2005). Each element of this example-vector consisted of the BOLD-response averaged across the time-window between 2 and 8 volumes post-stimulus onset, computed for each voxel of the feature-space. Subsequently, this value was baseline-corrected by considering the mean amplitude of the BOLD-response of the volumes immediately preceding the onset of the block (volumes $[-1,0]$ ), and subtracting this baseline from the original value.

After the computation of the 48 multidimensional examples, the classification procedure worked as follows. Examples were labeled according to their corresponding condition: person or category. They were randomly divided in two sets: a training set consisting of 40 examples ( 20 per condition) and a testing set made of 8 examples (4 per condition). For each participant individually, a linear SVM type-1 (Mourao-Miranda, Bokde, Born, Hampel, \& 
Stetter, 2005; Muller, Mika, Ratsch, Tsuda, \& Scholkopf, 2001) as implemented in BrainVoyager QX 2.1, was used to differentiate the two patterns of activation (person, category). The test subset was used to assess the accuracy of the trained classifier. This process was repeated 40 times for each participant with different train and test subsets, after which the average accuracy was computed and tested against chance level using a one-sample t-test. See figure 2.2 for a summary of the pattern classification approach used. 


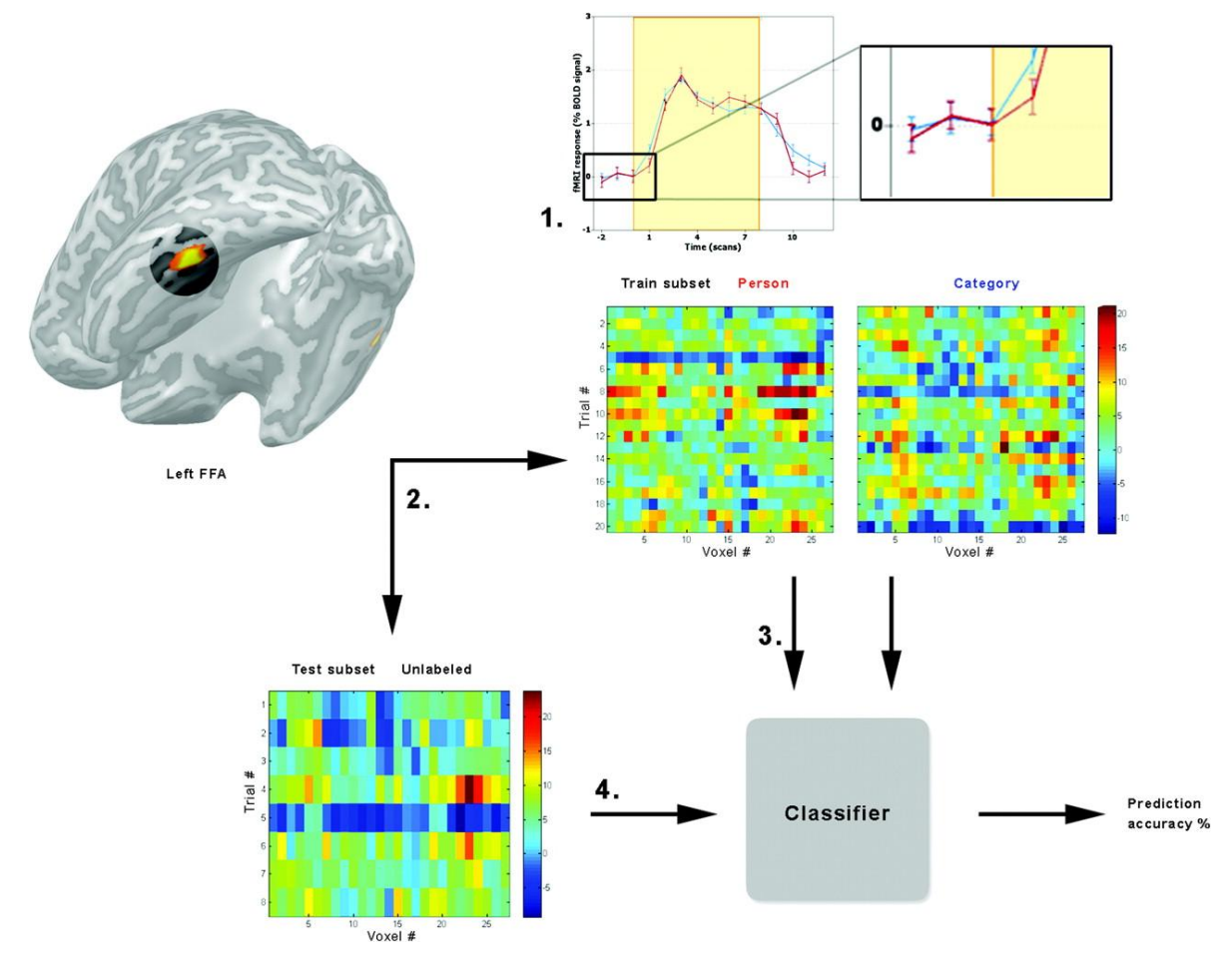

Figure 2.2. Summary of the analysis approach. 1. An event-related average plot shows the univariate contrast between person (red line) and category (blue line) context during the presentation of words (yellow interval. [0.8] volumes) in left FFA (defined by independent localizer). in which no significant difference was observed. As can be seen in the top right inset. the BOLD signal is at baseline prior to the presentation of the block of words, indicating no remaining effect of the instruction images on FFA activity. 2. The matrices represent the responses of the selected voxels ( $x$-axis) for all trials (y-axis). The trials are randomly assigned to a training set containing 20 labeled trials from each condition. and a test set with 4 unlabeled trials from both conditions. 3. A linear SVM is trained with the train set. 4. The accuracy of the classifier after training is assessed with the independent test set. The trials in the test set are not used in the training phase. guaranteeing an unbiased measure of the accuracy of the classifier after training. Steps 2 to 4 are iterated 40 times. after which the mean accuracy is computed and tested against chance level of $50 \%$. See text for details. 


\subsection{Results}

A univariate RFX GLM of the entire cortical volume was performed, but statistical analysis of the person > category contrast showed no active voxels both in the left and right hemisphere. A region of interest (ROI) analysis of both left and right FFA was subsequently carried out on the individual subjects (see table 2.2). FFA showed a clear response to the blocks of words, but this response was generally equal in both conditions. The statistical contrasts yielded no significant difference between the person and category contexts in any of the 6 left FFA's, nor in 4 of the right.

In contrast, the multivariate pattern analysis revealed a difference between conditions. Results from the pattern classification are depicted in table 2.3 and figure 2.3. Even though the overall level of activity in both conditions was the same, the pattern of activation across voxels in FFA did reflect the semantic context of the word blocks. The classifier was able to discriminate the response patterns in FFA that underlie the two semantic contexts significantly above chance level in all participants (see table 2.3). With one exception, all participants showed this distributed coding in FFA bilaterally. The multivoxel effect found in FFA is without directionality towards one of the two conditions. A classification accuracy that significantly deviates from chance level tells us that the patterns of responses elicited by the conditions are sufficiently distinct from each other to get noticed by the classifier. 
LFFA RFFA

\begin{tabular}{|c|c|c|c|c|}
\hline Subject & $\mathrm{t}$ & $p$ & $\mathrm{t}$ & $p$ \\
\hline 1 & 0.30 & 0.76 & 1.99 & 0.05 \\
\hline 2 & 1.22 & 0.22 & 0.77 & 0.14 \\
\hline 3 & -1.15 & 0.14 & 0.01 & 1 \\
\hline 4 & 0.93 & 0.35 & 1.45 & 0.15 \\
\hline 5 & 1.30 & 0.2 & 2.89 & 0.003 \\
\hline 6 & -0.69 & 0.49 & -0.76 & 0.45 \\
\hline
\end{tabular}

Table 2.2. GLM contrast (person>category) results per participant (1-6) separately for left and right FFA. 
LFFA

\begin{tabular}{|c|c|c|c|c|c|c|}
\hline Subject & $\%$ correct & $\mathrm{t}$ & $p$ & $\%$ correct & $\mathrm{t}$ & $p$ \\
\hline 1 & 62.2 & 4.78 & 0.0001 & 56.6 & 2.59 & 0.013 \\
\hline 2 & 60 & 4.37 & 0.0001 & 60 & 3.48 & 0.001 \\
\hline 3 & 60.9 & 3.90 & 0.0001 & 58.8 & 3.56 & 0.001 \\
\hline 4 & 55.9 & 2.38 & 0.022 & 47.2 & -1.01 & 0.317 \\
\hline 5 & 61.3 & 4.68 & 0.0001 & 59.4 & 3.72 & 0.001 \\
\hline 6 & 56.3 & 2.21 & 0.033 & 58.4 & 3.84 & 0.0001 \\
\hline
\end{tabular}

RFFA

Table 2.3. Classification accuracies per participant (1-6) separately for left and right FFA. 


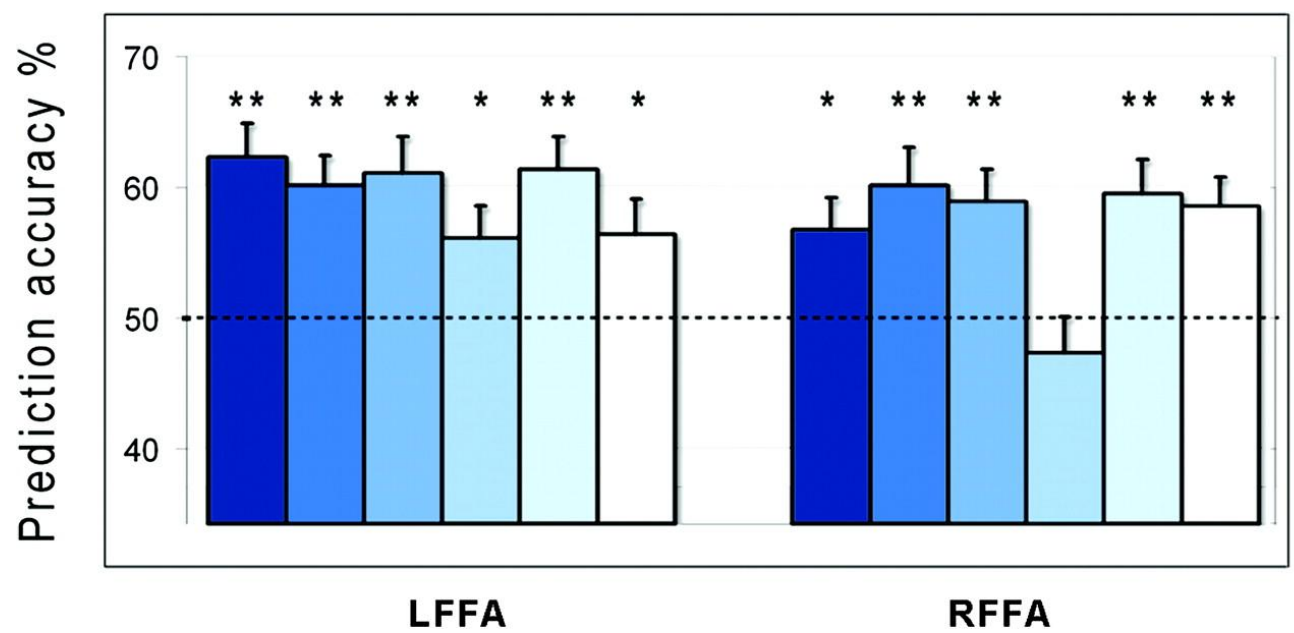

Figure 2.3. Resulting classification accuracies (\%) after 40 iterations per participant $(+S E M)$. depicted for left and right FFA. Average classification accuracies derived from FFA (bilaterally for all but one participant) show a significant deviation from chance. Different tints of blue represent different participants. Dashed line at 50\% (chance level). ${ }^{*} \mathrm{p}<0.05$. ** $\mathrm{p}<0.001$ 


\subsection{Discussion}

We aimed to further delineate the functional role of FFA in the context of semantic association about people. The present study investigated if FFA is able to discriminate between a person-specific and a categorical semantic context, while keeping visual input constant across conditions. Using an fMRI block design, we measured the response of FFA during presentation of blocks of words in which the words were either related in a categorical or a personal context. Both conditions elicited a relatively strong response in FFA, but univariate statistical contrasts yielded no difference in BOLD-signal between them within FFA or elsewhere. Nonetheless, multi-voxel pattern analysis revealed that activations in FFA do actually code for the semantic contexts used. This suggests that 1) multivariate pattern analysis was able to reveal neural coding of informational contents, where univariate analysis could show no differences between conditions - which has significant methodological implications - and 2) FFA codes for semantic contexts of word sets, presented free of direct face stimuli. In other words, although the words comprising the individual person profiles could be related to previously presented instructional face stimuli - no actual face stimuli were presented in the trials here analyzed. The fact that activity in FFA nonetheless was found to contain sufficient information about the semantic contexts of the trials, has significant and far-reaching implications for models about the role of FFA in face and identity coding. This we discuss in more detail below.

In light of the role of FFA in face identification (Andrews \& Ewbank, 2004; Barton, et al., 2002; Gauthier, et al., 2000; Grill-Spector, et al., 2004; Haxby, et al., 2002; Hoffman \& Haxby, 2000; Kriegeskorte, et al., 2007; Loffler, et al., 2005; Mur, et al., 2010), our findings illustrate - for the first time - the possibility that the coding of face identity in FFA goes beyond visual features and might include conceptual and biographical knowledge about a face in the absence of visual facial information.

In the present design we carefully attempted to equalize both conditions selecting the words belonging to each condition from the same word matrix. However, the instructional images we presented at the beginning of each block differed between conditions. As the neural responses elicited by the images were irrelevant for our hypothesis, we excluded the corresponding volumes from our analysis. We can also rule out the influence of the instruction over the block of words, and, in turn, on the contextual processing. The long interval between the instruction and the block of words $(10000 \mathrm{~ms})$ ensured that the hemodynamic 
response related to the instructional images returned to baseline before the block onset. This prevented spilling-over effects from the image (as can be seen in figure 2.2). This approach excluded any direct interference from the instruction with the activation pattern within in FFA during the run. Still, one could argue that FFA plays a role in the working memory of faces. From this perspective, the face that is presented prior to the block of words could potentially bias the activity in FFA in the person-specific condition, offering an alternative explanation for our findings. Several studies, however, have demonstrated that the maintenance of faces in working memory exhibit delay-period activation in FFA (Druzgal \& D'Esposito, 2003; Johnson, Mitchell, Raye, D'Esposito, \& Johnson, 2007; Postle, Druzgal, \& D'Esposito, 2003; Ranganath, DeGutis, \& D'Esposito, 2004). Our results show that the BOLD-signal in FFA returns to baseline in between the face presentation and the block of words, indicating no transient effect of working memory. In addition, an effect of face working memory on the activation of FFA would most likely result in a stronger BOLDsignal in the person-specific condition compared to the category condition, an effect that was not apparent in our data. These two points indicate that it is unlikely that the picture of the face is directly responsible for the difference between the two experimental conditions.

The exact source of the observed person vs. category effect in FFA, however, remains subject to discussion. It is possible that FFA receives topdown modulation from one or multiple areas from the semantic (person or category identification) network (Martin, 2007; Martin \& Chao, 2001) that were involved in the processing of the contexts during the task. However, since the univariate contrast between the person and category conditions did not reveal any areas of interest, the possible source of any modulatory effects remains subject to speculation. Several studies observed a wide variety of modulatory effects on FFA, ranging from modulation by face working memory load (Druzgal \& D'Esposito, 2001), likely via feedback from prefrontal cortex (Druzgal \& D'Esposito, 2003), task specificity (Chiu, Esterman, Han, Rosen, \& Yantis, 2010; Reddy, Moradi, \& Koch, 2007), face/non-face category expectation (Puri, Wojciulik, \& Ranganath, 2009) and language describing faces (Aziz-Zadeh, et al., 2008). Moreover, a study by von Kriegstein and colleagues demonstrated that familiar voices activate FFA during a speaker identification task (von Kriegstein, Kleinschmidt, Sterzer, \& Giraud, 2005). Based on what has been established about the role of anterior inferotemporal cortex (aIT) in retrieval of semantic knowledge and face identification (Brambati, et al., 2010; Evans, et al., 1995; Gainotti, et al., 2003; Grabowski, et al., 2001; Haxby, et al., 2000; Kriegeskorte, et al., 2007; Patterson, Nestor, \& Rogers, 2007; Sergent \& 
Signoret, 1992; Tranel, 2006) one might suspect this brain region to be involved in feeding back conceptual information to FFA. Still, it has to be taken into consideration that participants in the current study were provided with semantic information online during the task, which is in contrast with studies that had the participants study conceptual knowledge ahead of the scanning task, allowing learned information about identity to consolidate. It is therefore possible that aIT did not directly modulate FFA. The actual source might be part of the face identification network and that aIT acts as a semantic hub (Patterson, et al., 2007), communicating with other areas involved in perception. Future studies might investigate the functional or effective connectivity between FFA and other areas to resolve this debate, for instance with use of Granger causality mapping (Goebel, Roebroeck, Kim, \& Formisano, 2003; Roebroeck, Formisano, \& Goebel, 2005), dynamic causal modelling (Friston, Harrison, \& Penny, 2003) or methods based on mutual entropy (Hinrichs, Heinze, \& Schoenfeld, 2006).

Alternatively, the person vs category effect within FFA can be a local process within FFA and adjacent areas without input from temporal and frontal sites. In general, the early position of FFA in the visual system hierarchy (Haxby, et al., 1991) may suggest that FFA likely does not directly process semantics. But there are empirical estimates on the time course of conceptual encoding (Indefrey \& Levelt, 2004; Schmitt, Schiltz, Zaake, Kutas, \& Munte, 2001; Thorpe, Fize, \& Marlot, 1996) that estimates conceptual encoding starting around 0 to $150 \mathrm{~ms}$ after stimulus onset. ERP signatures related to facial feature and identity encoding are within this time window (N170 and N250) with estimated sources within OFA and FFA respectively (Bentin, Allison, Puce, Perez, \& McCarthy, 1996; Kaufmann, Schweinberger, \& Burton, 2009; Schweinberger, Pickering, Jentzsch, Burton, \& Kaufmann, 2002). So it may well be that facial encoding and conceptual/semantic access run in parallel. The access might take place in a distributed manner within face selective regions, as shown by Haxby and colleagues. They discussed the distributed representation of faces, cats and objects in human ventral temporal cortex (Haxby, et al., 2001). They show that the distinct pattern of responses associated with each of the categories is not exclusively due to regions that show maximal response to that category. In fact, when these regions are excluded from the analysis, looking at the patterns of responses of the remaining areas was sufficient to identify the category being watched by the participant. In what they termed object form topography, they illustrate that complex categorical and semantic features underlying visual objects might be represented by distributed patterns across category-specific regions. Hypothetically, presenting a semantic context related 
to one of these categories could elicit a spatially distinct pattern of responses that to some extent corresponds to the category the context refers to. 


\subsection{Conclusion}

In the current study, we compared the responses of bilateral FFA to words that were visually identical - but organized in different sets to define two conditions: a person context and a category context. Our results showed that multi-voxel pattern analysis could reveal differential coding in FFA for the two types of semantic associations (semantic contexts). Univariate analysis showed no difference in overall response. Overall, the data suggest that FFA already contains information about semantic context, even in the absence of face stimuli. This has strong implications for models of the role of FFA in semantic and identity coding. Future research should aim to reveal how FFA interacts with other regions related to semantic and memory processing, to uncover the mechanism of this differential coding. 


\subsection{Acknowledgements}

The authors would like to thank Valerie Goffaux and Russel Epstein for providing the stimuli for the FFA localizer and Tom de Graaf, Marieke Mur and Sanne Rutten for valuable discussions and comments on earlier versions of this manuscript. 


\subsection{References}

Andrews, T. J., \& Ewbank, M. P. (2004). Distinct representations for facial identity and changeable aspects of faces in the human temporal lobe. Neuroimage, 23(3), 905-913.

Aziz-Zadeh, L., Fiebach, C. J., Naranayan, S., Feldman, J., Dodge, E., \& Ivry, R. B. (2008). Modulation of the FFA and PPA by language related to faces and places. Soc Neurosci, 3(3-4), 229-238.

Barton, J. J., Press, D. Z., Keenan, J. P., \& O'Connor, M. (2002). Lesions of the fusiform face area impair perception of facial configuration in prosopagnosia. Neurology, 58(1), 71-78.

Bentin, S., Allison, T., Puce, A., Perez, E., \& McCarthy, G. (1996). Electrophysiological Studies of Face Perception in Humans. J Cogn Neurosci, 8(6), 551-565.

Brambati, S. M., Benoit, S., Monetta, L., Belleville, S., \& Joubert, S. (2010). The role of the left anterior temporal lobe in the semantic processing of famous faces. Neuroimage, 53(2), 674-681.

Bruce, V., \& Young, A. (1986). Understanding face recognition. Br J Psychol, 77 ( Pt 3), 305-327.

Chiu, Y. C., Esterman, M., Han, Y., Rosen, H., \& Yantis, S. (2010). Decoding Task-based Attentional Modulation during Face Categorization. J Cogn Neurosci.

Cox, D. D., \& Savoy, R. L. (2003). Functional magnetic resonance imaging (fMRI) "brain reading": detecting and classifying distributed patterns of fMRI activity in human visual cortex. Neuroimage, 19(2 Pt 1), 261-270.

Craigie, M., \& Hanley, J. R. (1993). Access to visual information from a name is contingent on access to identity-specific semantic information. Memory, 1(4), 367-391.

Craigie, M., \& Hanley, J. R. (1997). Putting faces to names. Br J Psychol, 88 ( Pt 1), 157-171. 
Druzgal, T. J., \& D'Esposito, M. (2001). Activity in fusiform face area modulated as a function of working memory load. Brain Res Cogn Brain Res, 10(3), 355-364.

Druzgal, T. J., \& D'Esposito, M. (2003). Dissecting contributions of prefrontal cortex and fusiform face area to face working memory. $J \operatorname{Cogn}$ Neurosci, 15(6), 771-784.

Evans, J. J., Heggs, A. J., Antoun, N., \& Hodges, J. R. (1995). Progressive prosopagnosia associated with selective right temporal lobe atrophy. A new syndrome? Brain, 118 ( Pt 1), 1-13.

Formisano, E., De Martino, F., Bonte, M., \& Goebel, R. (2008). "Who" is saying "what"? Brain-based decoding of human voice and speech. Science, 322(5903), 970-973.

Formisano, E., De Martino, F., \& Valente, G. (2008). Multivariate analysis of fMRI time series: classification and regression of brain responses using machine learning. Magn Reson Imaging, 26(7), 921-934.

Friston, K. J., Harrison, L., \& Penny, W. (2003). Dynamic causal modelling. Neuroimage, 19(4), 1273-1302.

Gainotti, G., Barbier, A., \& Marra, C. (2003). Slowly progressive defect in recognition of familiar people in a patient with right anterior temporal atrophy. Brain, 126(Pt 4), 792-803.

Gauthier, I., Tarr, M. J., Moylan, J., Skudlarski, P., Gore, J. C., \& Anderson, A. W. (2000). The fusiform "face area" is part of a network that processes faces at the individual level. J Cogn Neurosci, 12(3), 495-504.

Goebel, R., Esposito, F., \& Formisano, E. (2006). Analysis of functional image analysis contest (FIAC) data with brainvoyager QX: From singlesubject to cortically aligned group general linear model analysis and self-organizing group independent component analysis. Hum Brain Mapp, 27(5), 392-401.

Goebel, R., Roebroeck, A., Kim, D. S., \& Formisano, E. (2003). Investigating directed cortical interactions in time-resolved fMRI data using vector autoregressive modeling and Granger causality mapping. Magn Reson Imaging, 21(10), 1251-1261. 
Grabowski, T. J., Damasio, H., Tranel, D., Ponto, L. L., Hichwa, R. D., \& Damasio, A. R. (2001). A role for left temporal pole in the retrieval of words for unique entities. Hum Brain Mapp, 13(4), 199-212.

Grill-Spector, K., Knouf, N., \& Kanwisher, N. (2004). The fusiform face area subserves face perception, not generic within-category identification. Nat Neurosci, 7(5), 555-562.

Haxby, J. V., Gobbini, M. I., Furey, M. L., Ishai, A., Schouten, J. L., \& Pietrini, P. (2001). Distributed and overlapping representations of faces and objects in ventral temporal cortex. Science, 293(5539), 2425-2430.

Haxby, J. V., Grady, C. L., Horwitz, B., Ungerleider, L. G., Mishkin, M., Carson, R. E., et al. (1991). Dissociation of object and spatial visual processing pathways in human extrastriate cortex. Proc Natl Acad Sci U $S$ A, 88(5), 1621-1625.

Haxby, J. V., Hoffman, E. A., \& Gobbini, M. I. (2000). The distributed human neural system for face perception. Trends Cogn Sci, 4(6), 223-233.

Haxby, J. V., Hoffman, E. A., \& Gobbini, M. I. (2002). Human neural systems for face recognition and social communication. Biol Psychiatry, 51(1), 59-67.

Haynes, J. D., \& Rees, G. (2006). Decoding mental states from brain activity in humans. Nat Rev Neurosci, 7(7), 523-534.

Hinrichs, H., Heinze, H. J., \& Schoenfeld, M. A. (2006). Causal visual interactions as revealed by an information theoretic measure and fMRI. Neuroimage, 31(3), 1051-1060.

Hoffman, E. A., \& Haxby, J. V. (2000). Distinct representations of eye gaze and identity in the distributed human neural system for face perception. Nat Neurosci, 3(1), 80-84.

Indefrey, P., \& Levelt, W. J. (2004). The spatial and temporal signatures of word production components. Cognition, 92(1-2), 101-144.

Johnson, M. R., Mitchell, K. J., Raye, C. L., D'Esposito, M., \& Johnson, M. K. (2007). A brief thought can modulate activity in extrastriate visual 
areas: Top-down effects of refreshing just-seen visual stimuli. Neuroimage, 37(1), 290-299.

Kamitani, Y., \& Tong, F. (2005). Decoding the visual and subjective contents of the human brain. Nat Neurosci, 8(5), 679-685.

Kanwisher, N., McDermott, J., \& Chun, M. M. (1997). The fusiform face area: a module in human extrastriate cortex specialized for face perception. $J$ Neurosci, 17(11), 4302-4311.

Kaufmann, J. M., Schweinberger, S. R., \& Burton, A. M. (2009). N250 ERP correlates of the acquisition of face representations across different images. J Cogn Neurosci, 21(4), 625-641.

Kriegeskorte, N., Formisano, E., Sorger, B., \& Goebel, R. (2007). Individual faces elicit distinct response patterns in human anterior temporal cortex. Proc Natl Acad Sci U S A, 104(51), 20600-20605.

Loffler, G., Yourganov, G., Wilkinson, F., \& Wilson, H. R. (2005). fMRI evidence for the neural representation of faces. Nat Neurosci, 8(10), 1386-1390.

Martin, A. (2007). The representation of object concepts in the brain. Annu Rev Psychol, 58, 25-45.

Martin, A., \& Chao, L. L. (2001). Semantic memory and the brain: structure and processes. Curr Opin Neurobiol, 11(2), 194-201.

Mourao-Miranda, J., Bokde, A. L., Born, C., Hampel, H., \& Stetter, M. (2005). Classifying brain states and determining the discriminating activation patterns: Support Vector Machine on functional MRI data. Neuroimage, 28(4), 980-995.

Muller, K. R., Mika, S., Ratsch, G., Tsuda, K., \& Scholkopf, B. (2001). An introduction to kernel-based learning algorithms. IEEE Trans Neural Netw, 12(2), 181-201.

Mur, M., Bandettini, P. A., \& Kriegeskorte, N. (2009). Revealing representational content with pattern-information fMRI--an introductory guide. Soc Cogn Affect Neurosci, 4(1), 101-109. 
Mur, M., Ruff, D. A., Bodurka, J., Bandettini, P. A., \& Kriegeskorte, N. (2010). Face-identity change activation outside the face system: "release from adaptation" may not always indicate neuronal selectivity. Cereb Cortex, 20(9), 2027-2042.

Norman, K. A., Polyn, S. M., Detre, G. J., \& Haxby, J. V. (2006). Beyond mindreading: multi-voxel pattern analysis of fMRI data. Trends Cogn Sci, 10(9), 424-430.

Patterson, K., Nestor, P. J., \& Rogers, T. T. (2007). Where do you know what you know? The representation of semantic knowledge in the human brain. Nat Rev Neurosci, 8(12), 976-987.

Pereira, F., Mitchell, T., \& Botvinick, M. (2009). Machine learning classifiers and fMRI: a tutorial overview. Neuroimage, 45(1 Suppl), S199-209.

Postle, B. R., Druzgal, T. J., \& D'Esposito, M. (2003). Seeking the neural substrates of visual working memory storage. Cortex, 39(4-5), 927-946.

Puce, A., Allison, T., Gore, J. C., \& McCarthy, G. (1995). Face-sensitive regions in human extrastriate cortex studied by functional MRI. J Neurophysiol, 74(3), 1192-1199.

Puri, A. M., Wojciulik, E., \& Ranganath, C. (2009). Category expectation modulates baseline and stimulus-evoked activity in human inferotemporal cortex. Brain Res, 1301, 89-99.

Ranganath, C., DeGutis, J., \& D'Esposito, M. (2004). Category-specific modulation of inferior temporal activity during working memory encoding and maintenance. Brain Res Cogn Brain Res, 20(1), 37-45.

Reddy, L., Moradi, F., \& Koch, C. (2007). Top-down biases win against focal attention in the fusiform face area. Neuroimage, 38(4), 730-739.

Roebroeck, A., Formisano, E., \& Goebel, R. (2005). Mapping directed influence over the brain using Granger causality and fMRI. Neuroimage, 25(1), 230-242.

Schmitt, B. M., Schiltz, K., Zaake, W., Kutas, M., \& Munte, T. F. (2001). An electrophysiological analysis of the time course of conceptual and 
syntactic encoding during tacit picture naming. J Cogn Neurosci, 13(4), 510-522.

Schweinberger, S. R., Pickering, E. C., Jentzsch, I., Burton, A. M., \& Kaufmann, J. M. (2002). Event-related brain potential evidence for a response of inferior temporal cortex to familiar face repetitions. Brain Res Cogn Brain Res, 14(3), 398-409.

Sergent, J., Ohta, S., \& MacDonald, B. (1992). Functional neuroanatomy of face and object processing. A positron emission tomography study. Brain, 115 Pt 1, 15-36.

Sergent, J., \& Signoret, J. L. (1992). Implicit access to knowledge derived from unrecognized faces in prosopagnosia. Cereb Cortex, 2(5), 389-400.

Thorpe, S., Fize, D., \& Marlot, C. (1996). Speed of processing in the human visual system. Nature, 381(6582), 520-522.

Tranel, D. (2006). Impaired naming of unique landmarks is associated with left temporal polar damage. Neuropsychology, 20(1), 1-10.

Tranel, D., Damasio, H., \& Damasio, A. R. (1997). A neural basis for the retrieval of conceptual knowledge. Neuropsychologia, 35(10), 13191327.

von Kriegstein, K., Kleinschmidt, A., Sterzer, P., \& Giraud, A. L. (2005). Interaction of face and voice areas during speaker recognition. J Cogn Neurosci, 


\section{Chapter 3}

\section{FFA - Precuneus Interaction During Processing of Words in Biographic Context ${ }^{2}$}

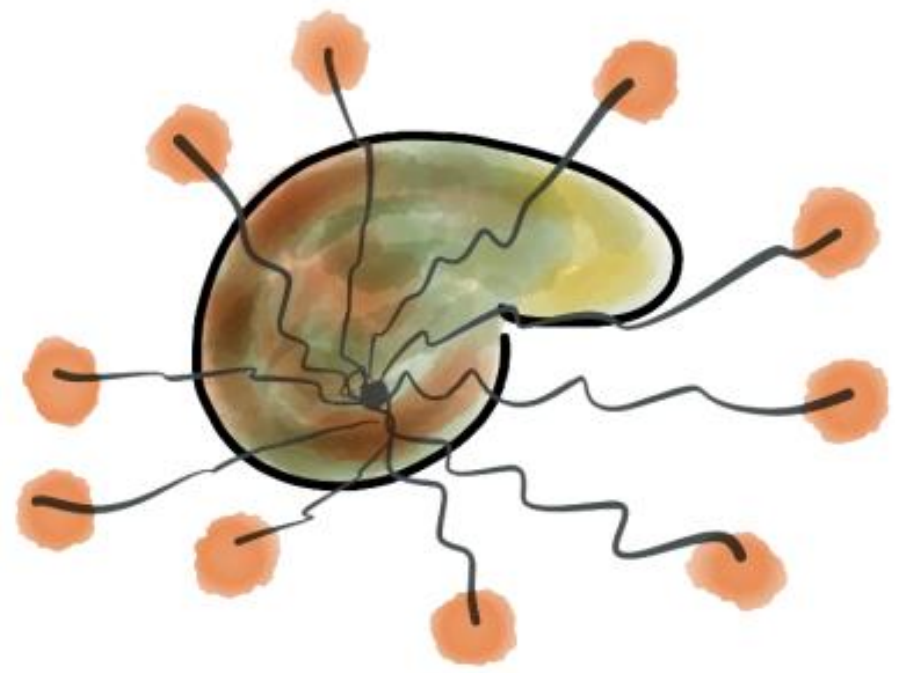

${ }^{2}$ Van den Hurk, J., Laufer, S. T., \& Jansma, B. M. FFA - Precuneus Interaction During Processing of Words in Biographic Context. Submitted. 


\section{Abstract}

When we identify a familiar face, our brain both processes the familiar visual features and the semantic knowledge we have about an individual. The Fusiform Face Area (FFA), a cortical region specifically dedicated to the processing of faces, has generally been studied in the context of visual face identity, neglecting the important conceptual part. Recently, we addressed this issue by presenting participants with words instead of faces. We created a personal (PER) and categorical (CAT) semantic condition from the same 8 × 8 word matrix, thereby instantly controlling for visual input across conditions. Conventional univariate analysis methods yielded no significant differences between the conditions across the brain. Nevertheless, multivoxel pattern analysis revealed that brain activity in FFA could significantly distinguish between the personal and categorical semantic conditions. However, it remained unclear how FFA obtained this background information, i.e. which regions/networks engage in functional interaction with FFA when personal semantic information related to faces is processed. In the current study we extended our previous design and dataset and analysis to address this issue. Using a multivariate searchlight algorithm, we identified four cortical regions that were sensitive to the context manipulation: the left precuneus, left inferior frontal gyrus (LIFG) and bilateral angular gyri. A custom version of psychophysiological interaction (PPI) analysis then showed that processing of semantic context is related to a functional coupling between bilateral FFA and the precuneus, and between FFA and right angular gyrus. The fact that FFA engages in functional interactions with a network underlying the processing of semantic context, even in the absence of a face, has substantial implications for conceptions of the role of FFA. 


\subsection{Introduction}

In everyday social situations, people are highly skilled in the recognition and consequent identification of observed faces. As soon as we see a familiar face, our brain recognizes it by matching the observed facial features to stored templates, after which access to biographical knowledge about the face is granted (Bruce \& Young, 1986). This process has shown to be remarkably efficient (Bentin, Allison, Puce, Perez, \& McCarthy, 1996; Bentin \& Deouell, 2000; Eimer, 2000; Henson, et al., 2003). Several studies have suggested that the Fusiform Face Area or FFA (Kanwisher, McDermott, \& Chun, 1997; Puce, Allison, Gore, \& McCarthy, 1995), a cortical module that prefers faces over other visual categorical stimuli, also plays an important role in the identification of faces (Andrews \& Ewbank, 2004; Barton, Press, Keenan, \& O'Connor, 2002; Gauthier, et al., 2000; Grill-Spector, Knouf, \& Kanwisher, 2004; Haxby, Hoffman, \& Gobbini, 2002; Hoffman \& Haxby, 2000; Loffler, Yourganov, Wilkinson, \& Wilson, 2005), possibly predominantly in the right hemisphere (Ma \& Han, 2011; Verosky \& Turk-Browne, 2012). However, the identity of a familiar face comprises not only visual features but also biographical knowledge. It is not straightforward to experimentally disentangle these factors, and the majority of studies investigating the neural processing of identity have not separated visual and semantic face identity (but see Mur, Ruff, Bodurka, Bandettini, \& Kriegeskorte, 2010). In a recent study, we addressed this issue, presenting participants with blocks of words, either in a person ('biographical') context or in a categorical ('non-biographical') context. We could show that FFA is able to discriminate between these two distinct semantic contexts, without simultaneous presentation of face stimuli (van den Hurk, Gentile, \& Jansma, 2011; see Chapter 2). Although these findings shed a new light on the functional role of FFA in the processing of identity, they left open some fundamental questions. Primarily, how does FFA obtain this information on semantic (biographical) background? Are other regions in the brain sensitive to the distinction between person-related and categorical presentation of the same set of words? Does FFA interact with these regions during processing of semantic background information, and more importantly: is this interaction different for the person and the category context? The results from the previous study did not allow for any speculation about FFA 'preferring' the person context over the category context. Therefore, a specific stronger coupling between FFA and other cortical regions during the person context would provide important evidence for the perspective that FFA is indeed engaged during the processing of semantic identity information. 
The current study addressed these issues. In the scanner, participants were presented with blocks of 8 words in a person-related (PER) and a categoryrelated (CAT) context. Both conditions were created by using identical items, thereby immediately controlling for visual input across conditions (see table 3.1 and figure 3.1 for an overview). Next to univariate (GLM) fMRI statistics, we analyzed the data using a multivariate "searchlight" (Kriegeskorte, Goebel, \& Bandettini, 2006). In addition, we localized bilateral FFA for each subject. We hypothesized that 1) the searchlight algorithm reveals local patterns of voxels throughout the brain that carry information about the person-category context manipulation where univariate statistical methods probably may not, and 2) (a subset of) these regions show functional coupling with FFA during the task. More specifically, assuming that FFA is playing a role in the identification of faces, we hypothesized to find a stronger coupling between FFA and these target regions in the PER condition than in the CAT condition. 


\subsection{Materials and methods}

\subsubsection{Participants}

Ten right-handed healthy adults with normal vision took part in this study (six females, mean age 25.9 years old). Participants were screened for fMRI compatibility, signed informed-consent and were financially compensated for their participation. The study was approved by the ethical committee of the Faculty of Psychology and Neuroscience.

\subsubsection{Stimuli}

The stimuli, experimental procedure and data acquisition parameters were identical to the design used by Van den Hurk and colleagues (2011; see Chapter 2 ). We created two conditions from an $8 * 8$ word matrix (table 3.1), consisting of 8 categories (professions, capital cities, house types, sports, hobbies, domestic animals, music styles and car brands). The words used were all Dutch, which was the native language of all participants. For the category (CAT) condition, 8 blocks each consisting of 8 words were created by using the items from a single category per block (the columns in the word matrix). Each CAT block thus represented 1 of 8 categories (i.e. "professions"). For the person (PER) condition, 8 blocks each consisting of 8 words were created by using one word from each category (the rows in the word matrix): each resulting sequence of 8 words could be interpreted as a keyword description of an individual profile (i.e. a surgeon living in Amsterdam, playing soccer etc.). By using this approach, we were able to create two distinct semantic contexts while controlling for visual and semantic input across conditions.

For the person and category condition, we used 8 different face images (provided by Mark Steyvers http://psiexp.ss.uci.edu/research/software.htm) and 8 images of objects (softball glove, house, car, book, dog, construction worker, globe and saxophone) as instruction for the person and category conditions respectively. 


Toyota Amsterdam Surgeon $\begin{aligned} & \text { Terraced } \\ & \text { house }\end{aligned}$ Soccer Cooking Dog Ska

Hyundai Brussels Baker Apartment* Hockey Painting Cat Rock

Peugeot Paris Teacher Penthouse Ice skating Fishing Rabbit $\begin{aligned} & \text { Classical } \\ & \text { music }\end{aligned}$

Opel London Composer Villa Athletics Gardening Parakeet Pop

Suzuki Berlin Engineer Studio* Tennis Traveling Chicken House

Mercedes Madrid Fitter $\quad \begin{aligned} & \text { Travel } \\ & \text { trailer }\end{aligned} \quad$ Badminton $\begin{aligned} & \text { Puzzle } \\ & \text { solving }\end{aligned} \quad$ Hamster Jazz

Renault Lissabon Barber Apartment Rowing Reading Goldfish Soul building

Rover Stockholm Editor $\begin{aligned} & \text { House on Cricket Walking Lizard Blues } \\ & \text { corner* }\end{aligned}$

Table 3.1. $8 * 8$ word stimulus matrix. The two semantic contexts used in this experiment were derived from this stimulus matrix. Both the category and person condition consisted of 8 blocks of 8 words, where each block was created by taking all 8 words from a column (category context, i.e. "professions") or by taking 1 word from each column (person context, i.e. a surgeon living in Amsterdam, playing soccer etc.). The matrix has been translated to English for this article. Some words that represent different concepts in Dutch could not be translated literally (marked with an asterisk). 


\subsubsection{Procedure}

The experiment consisted of three runs in a block design. Each run consisted of 8 blocks from each condition (CAT, PER), and started with a fixation cross lasting for 26 seconds. At the beginning of each block an instructional image (face or object) was presented for 6 seconds. The image was followed by a fixation cross that lasted for 10 seconds. This interval allowed the BOLD response elicited by the stimulus in the instruction phase to return back to baseline before the onset of the actual block. Next, a block of 8 words was presented, where each word lasted for $1500 \mathrm{~ms}$, followed by $500 \mathrm{~ms}$ of fixation (see figure 3.1). Participants were instructed to pay close attention to the block of words and to associate each of them with the image in the instruction (i.e. to associate the category sports with the image of a softball glove, within the category condition). The intention of this approach was to encourage the participants to actively create a semantic context out of the presented words they were facing. This in turn ensured that the block of words were not merely processed as a within category (CAT) vs a between category (PER) condition. They were informed that after each run, all images were presented one by one. The task of the participants was to recall as many word as possible related to that category or person. This additional debriefing phase was included to encourage participant to attend to the block of words inside the scanner. 


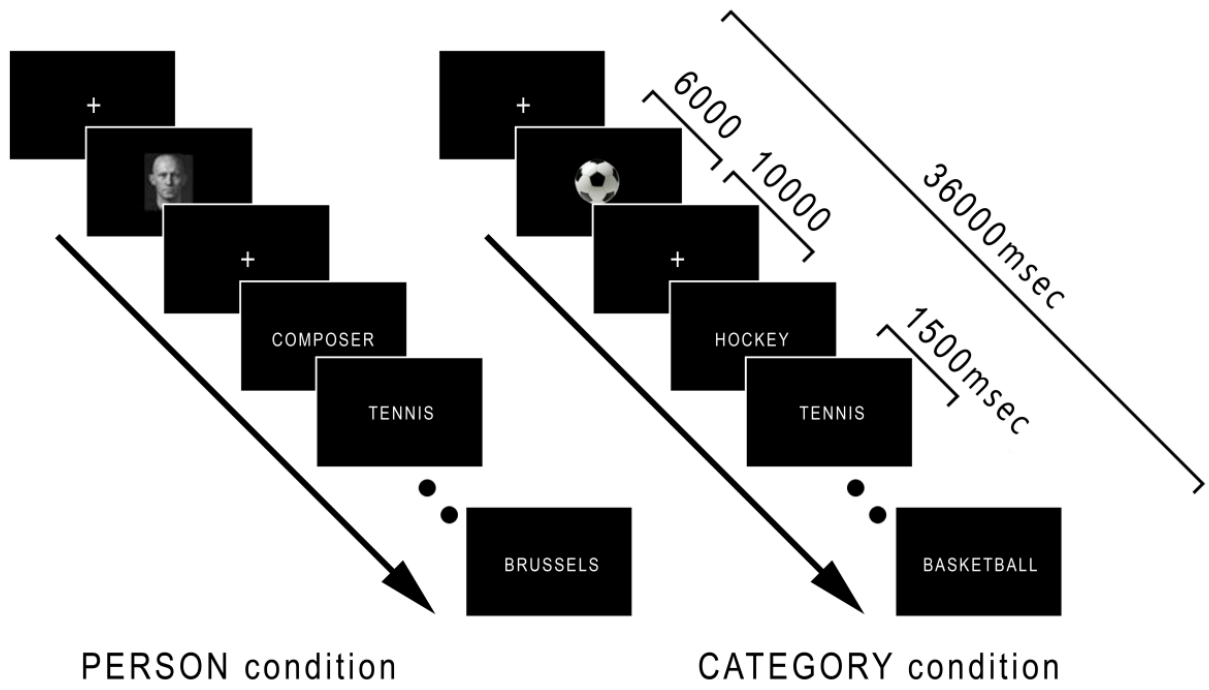

Figure 3.1. Experimental design used. The figure illustrates one block of both conditions. A block started with a fixation period, follow by an instructional image of a face (left) or object (right) for $6 \mathrm{~s}$. The image indicated which of the 2 contexts was about to be presented. The instruction "person" is given by a face, followed by a list of 8 words associated to the person's background (a composer who plays tennis as a hobby and lives in Brussels). The instruction "category" is given by an object (here a ball referring to category sports), followed by a list of 8 words from that category. The participant was instructed to associate the 8 words with the instructional image. To check if the participant was paying attention during the scanning, after each run, he or she was presented with all the instructional images and asked to recall as many keywords he or she remembered in combination with each image.

\subsubsection{Data acquisition}

Functional and anatomical images were acquired on a 3T Siemens Magnetom Allegra head scanner (Siemens Medical System, Erlangen, Germany) using a standard head coil. Thirty-two oblique axial slices $(3.5 \mathrm{~mm} \times 3.5 \mathrm{~mm}$, slice thickness $3,5 \mathrm{~mm}$, interslice distance $0 \mathrm{~mm}$ ) covering the entire cortical volume were collected using a standard echo planar-imaging (EPI) sequence (repetition time $[\mathrm{TR}]=2 \mathrm{sec}$, matrix size $=64 \times 64$, echo time $[\mathrm{TE}]=30 \mathrm{~ms})$. For each run, 
we collected 355 functional volumes, of which the first 2 were excluded due to $\mathrm{T} 1$ saturation. In addition to the experimental runs, an independent FFA localizer run was included. Anatomical images covering the whole brain were obtained after the experimental runs but before the localizer, using a $1 \times 1 \times 1 \mathrm{~mm}$ resolution T1-weighted ADNI sequence $([\mathrm{TR}]=2250 \mathrm{~ms}$; $[\mathrm{TE}]=2.6 \mathrm{~ms}$; flip angle $=9^{\circ}$ ).

The participants were placed comfortably in the scanner and their head was fixed with soft foam pads. Participants saw the stimuli projected on a screen through a mirror mounted on the head coil. Stimulus presentation was synchronized with MR data acquisition by triggering the stimulus program (Presentation software, Neurobehavioral Systems Inc, Albany, CA) with the first MR pulse.

\subsubsection{Analysis}

Functional and anatomical data were pre-processed and analysed using the BrainVoyager QX 2.2 package (Brain Innovation, Maastricht, The Netherlands). Functional volumes were first corrected for slice scan-time differences and 3D head motion using 3 translation and 3 rotation parameters. In order to enhance the subsequent alignment of the functional images to the anatomical volume, the first and second runs were corrected with the third run as intra-session reference, as the acquisition of this run was temporally adjacent to the anatomical scan. Subsequently, linear trends and low frequency temporal drifts were removed from the data using a high-pass filter, removing temporal frequencies below 2 cycles per run. After the pre-processing, functional data were co-registered to the high-resolution anatomical volume and normalized to Talairach space.

For each participant, the location of the Fusiform Face Area was determined bilaterally by an independent localizer run, using grey scale images of faces, scrambled faces and houses. The procedure defines the FFA as the result of a conjunction analysis (FDR-corrected at $q=0.05$ ) between a face responsive contrast (\%BOLD signal change faces $>$ scrambled faces) and a face selective contrast (\%BOLD signal change faces $>$ houses).

Conventional univariate statistical analysis of functional was performed on an average cortical surface, obtained by reconstructing both hemispheres for each participant. The surfaces were subsequently aligned using a cortex-based alignment procedure, an algorithm that uses individual curvature information to align corresponding gyri and sulci across participants (Goebel, Esposito, \& Formisano, 2006). The analysis was performed using a random effects GLM, 
using subject as random variable. A design matrix was created, using a predictor for both conditions. The predicted time courses were adjusted for the typical hemodynamic response delay by convolution with a canonical (double gamma) hemodynamic response function (HRF). Statistical contrasts were considered at the whole-brain level (FDR corrected at $q=0.05$ ).

On top of looking at the univariate activation differences between the two conditions, the data was also inspected on differences in multivariate information between the conditions using a multivariate searchlight approach (Kriegeskorte \& Bandettini, 2007; Kriegeskorte, et al., 2006). This descriptive method uses a spherical 'searchlight' that centers on every voxel of the cortical volume and gives an estimate of the presence of information in the spherical surround. On a single subject level, a GLM contrast between two conditions is fed to the algorithm. The $t$-values of this contrast are squared at each voxel, after which the algorithm visits all voxels individually and averages the squared $t$ values within the searchlight sphere with predefined radius surrounding the centered voxel (Kriegeskorte, et al., 2006). This approach provides a multivariate effect measure to indicate the difference between the activity patterns of the two conditions within the searchlight's sphere. Using this method, a whole-brain map is obtained that shows regions where multivariate information about the conditions is available. In our analysis, we used the searchlight algorithm as implemented in BrainVoyager QX 2.2 with a spherical radius of 4 voxels. For each subject individually, an information-based map was created. To account for between-subject variability and to increase statistical power, the single-subject maps were subsequently combined into an averaged information-based map. This resulting map was statistically corrected for multiple comparisons (Bonferroni $p<0.05$ ) and the resulting clusters were considered as target regions-of-interest (ROIs) in the subsequent connectivity analysis. In addition to these target regions, a randomly selected frontal (Talairach coordinates of center: $\mathrm{x}=0, \mathrm{y}=49, \mathrm{z}=-4$ ) and a parietal (Talairach coordinates of center: $\mathrm{x}=8, \mathrm{y}=$ $23, \mathrm{z}=67)$ cortical cluster of $10 \mathrm{~mm}$ isotropic each were selected as control regions.

In order to investigate the functional connectivity between FFA and the target regions, we used a method with an approach that is comparable to the 'psychophysiological interaction (PPI) analysis' (Friston, et al., 1997). The method investigates whether the correlation in brain activity between two cortical regions differs between two different cognitive states. It specifically looks for a statistical interaction between a) the strength of a functional connection between two regions and b) two different psychological states. In our experiment, we were interested in the functional connectivity between bilateral 
FFA and the target regions identified by the searchlight. More specifically, we hypothesized that in the light of the functional role of FFA, the functional coupling in the person context would be stronger between FFA and one or more target regions than in the category context. To exclude covariation in the BOLD signal between regions that is purely based on shared task input, we aimed to focus our custom PPI analysis solely on the signal fluctuations after the BOLDsignal had reached its peak and before it decreased back to baseline. The rationale behind this approach is that after a few seconds after block onset, the BOLD signal will reach its peak at which it will remain until block offset. The signal variations on this BOLD plateau are largely contributable to specific processes related to the semantic contexts used, corrupted by scanner noise. By consecutively segmenting the BOLD plateaus of each block into conditionspecific time series, we have the means to accurately investigate the processing of the semantic contexts. We therefore segmented the time courses, spatially averaged across voxels of a region, in condition specific time series. For each block, the time windows between 2 and 10 volumes post block onset (based on the length of the block ( 8 TR), taking the hemodynamic lag into account) were segmented. Each segment was zero-centered and then concatenated to the other segments of the corresponding condition. This process was repeated for each region as defined by the searchlight and resulted in PER- and CAT-specific time series, all with a length of 3 runs $* 8$ blocks $* 8$ volumes $=192$ time points. See figure 3.2 for an overview of this segmentation process. We also created condition-specific time series for left and right FFA. 


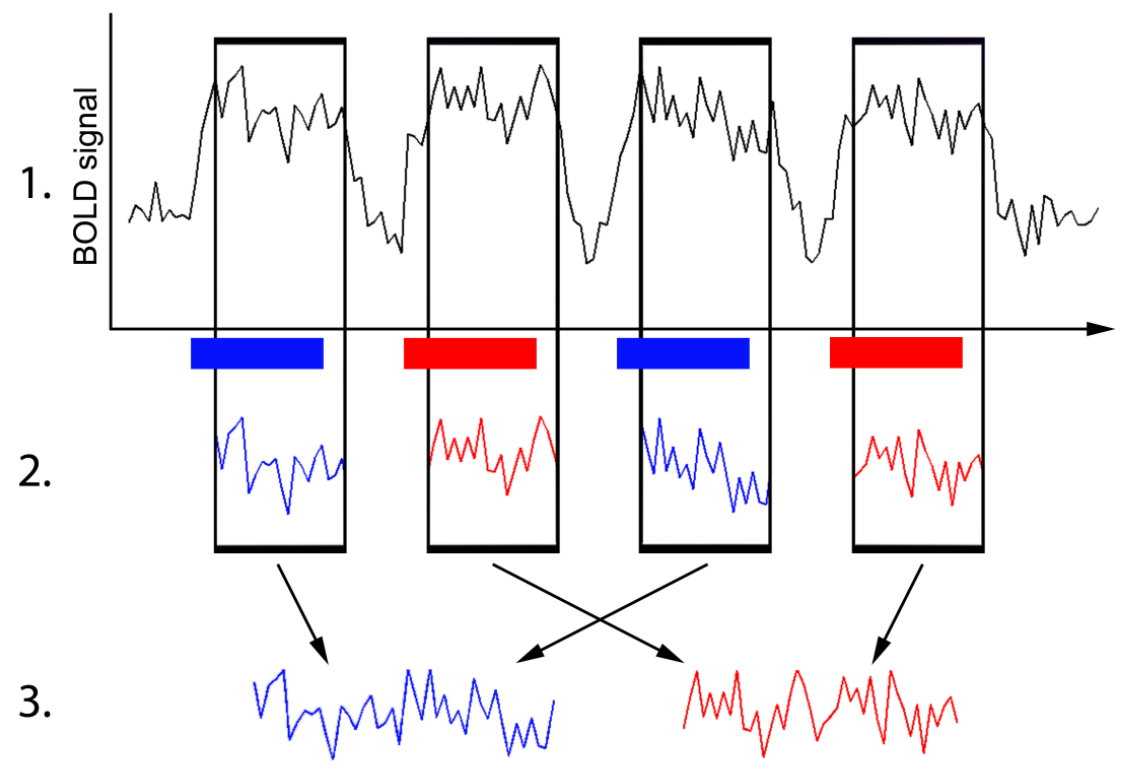

Figure 3.2. Creating condition-specific time series. 1. Example of a simulated time course of a region. BOLD-signal is modulated by both the category (blue) and person (red) conditions as illustrated by the colored bars. 2. The measured signal is segmented in epochs of 8 volumes each. The segmentation window starts with 2 volumes after block onset and ends with volume 10 after block onset. Each acquired segment is subsequently zero-centered by subtracting its mean. 3. Zero-centered segments are concatenated to the other segments from the corresponding condition, resulting in two time series, one for each condition. See text for more details.

Temporal correlation coefficients were computed between the time course from left and right FFA and from the target regions for the PER and CAT time series independently. Before statistically inspecting the results, we applied a variance stabilizing Fisher transformation (Fisher, 1915) on the computed correlation coefficients by transforming them with an inverse hyperbolic tangent function. This transformation leads to an correction of variance inhomogeneity and results in approximate normal distribution of the coefficients, making them suitable for statistical analysis. All computations were carried out using MATLAB R2008a (Mathworks Inc, Natick, MA). Finally, the results were analyzed using a repeated measures GLM with factors FFA (2) * condition (2) * target region (6). To account for a possible violation of the sphericity assumption, the degrees of freedom were adjusted using the Greenhouse-Geisser 
epsilon correction. The subsequent pair wise comparisons were computed using paired samples $t$-tests.

We also looked into the pair wise relationship between the 6 target regions by using the same approach. We computed the pair wise inter-region correlation per condition, transformed the coefficients and tested them using a repeated measures GLM. The pairs were compared using paired samples $t$-tests.

Although the approach we used to assess functional coupling is based on the procedure of PPI analysis, it differs on two important points from the original procedure. First, we used predefined regions of interest and estimated the functional coupling between them instead of the traditional approach, i.e. computing the interaction coefficients for each functional voxel in the brain with respect to a seed region. By localizing the target regions independently of the PPI analysis, we created an a priori assumption on the brain regions involved rather than performing blind mapping. This allows for a more focused interpretation of the results with regard to the networks of interest. Second, we only investigated the extent to which the activity in FFA and the target regions covary on the BOLD plateau, thus during the processing of the semantic context, instead of focusing on the entire time course. By ignoring modulations unrelated to the processing of the semantic contexts as much as possible, the PPI analysis is purely focusing at - and thus more sensitive to - the effects of interest. 


\subsection{Results}

The same words from an $8 \times 8$ matrix were presented to subjects in two conditions, in the PER (semantic person-related 'biographical') condition words described a person while in the CAT (categorical 'non-biographical') condition words form a specific category were presented. Figure 3.3 depicts the response in a participants' left FFA to the experimental conditions and shows that, although the responses to the instructional images (a face in the PER condition vs an object in the CAT condition) are different, the responses to the semantic contexts look very similar. It also illustrates that the BOLD-signal drops to baseline level before the start of the block of words. A subsequent univariate random-effects analysis on the entire brain did not reveal any differences between the two.

The searchlight procedure identified four clusters of voxels that contained information about the two conditions. Figure 3.4 shows these clusters projected on an averaged cortex reconstruction (Goebel, et al., 2006) at $p=0.05$ (Bonferroni corrected). Cluster A in figure 3.4 is located on the medial wall of the left parietal lobe, a region commonly referred to as the precuneus or ventral precuneus (Zhang \& Li, 2012). The mapped cluster extends ventrally toward the retrosplenial cortex and the posterior cingulated gyrus. Cluster B is located on the left inferior frontal gyrus, while clusters C and D were observed within the left and right angular gyri, respectively. 

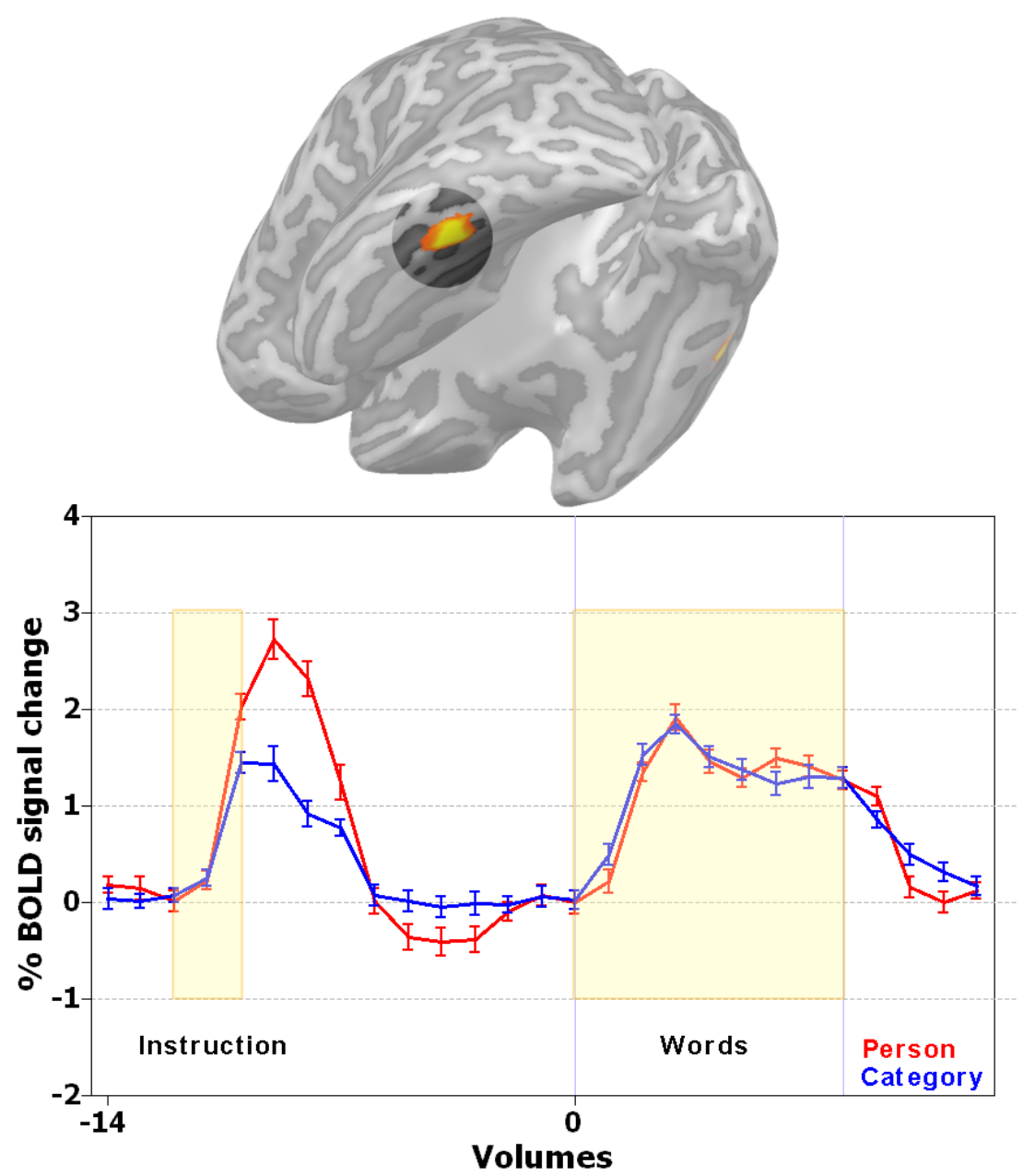

Figure 3.3. Example of FFA time course during experiment. The figure shows that FFA clearly responds stronger to the instructional image in the person condition (face) than in the category condition (object). After the initial response, the BOLD-signal returns to baseline in both conditions before the block of words commences. It can be seen that FFA responds to the words, but shows no obvious difference between the two conditions. 

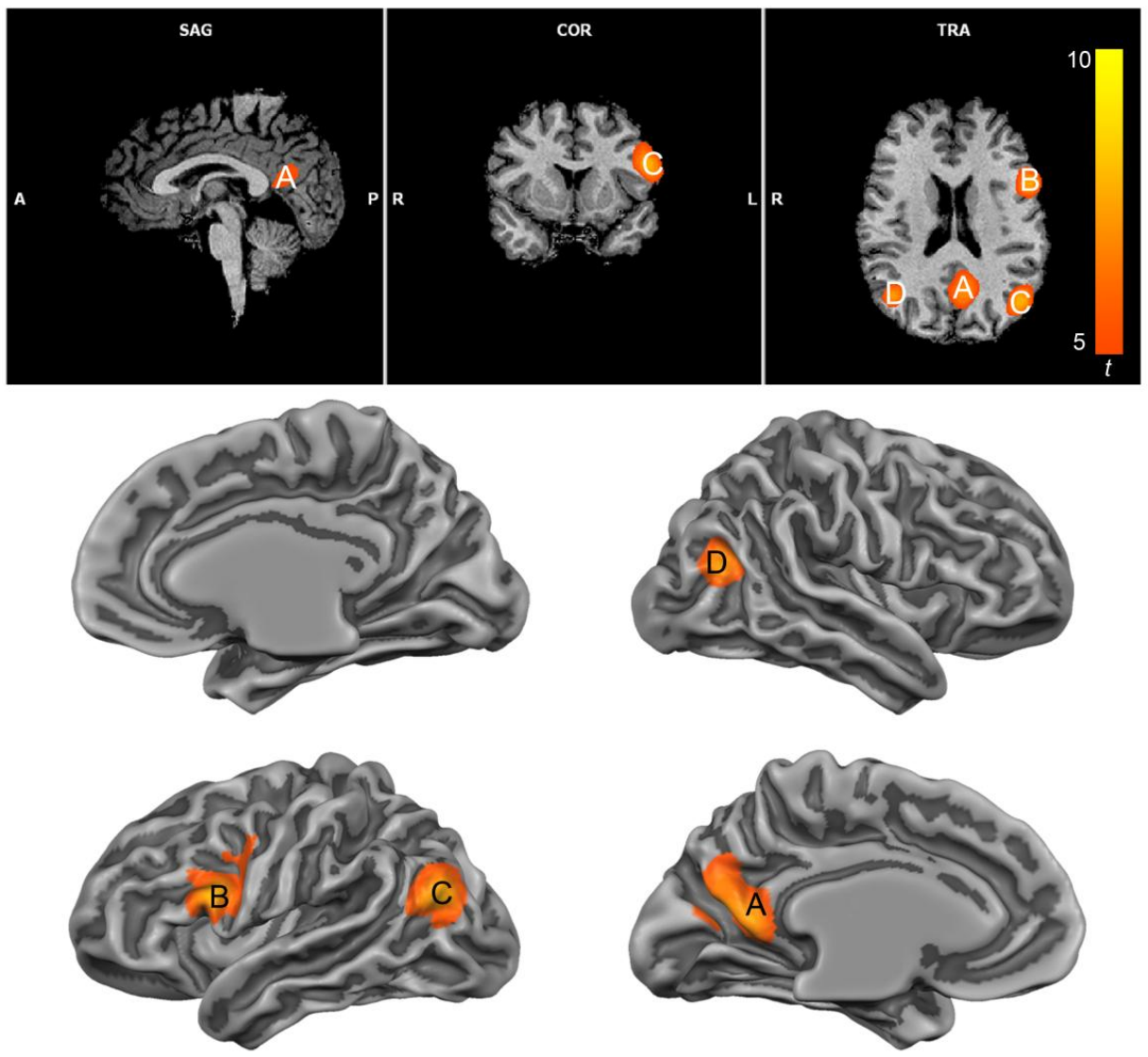

Figure 3.4. Searchlight results $(\mathbf{N}=10)$. Individual searchlight maps are combined into an average group map and corrected for multiple comparisons (Bonferroni, $p<0.05$ ). The resulting group maps are projected on a normalized (Talairach) volume (top) and on a cortex-based aligned average cortex reconstruction (bottom). The depicted clusters are located on (by approximation): A. left precuneus, B. left inferior frontal gyrus, C. left angular gyrus and D. right angular gyrus. Note that the top images are in radiological convention.

Based on the assumptions of the searchlight approach, we can state that processing in these four clusters (referred to as 'target regions' below) was different for words presented in a semantic person-related context as compared to a categorical context. In turn, this suggests that corresponding neural areas are sensitive to our experimental contextual manipulation. Because of this, we were interested to see if these contextually sensitive target regions in fact interacted with FFA. 
We performed a custom PPI analysis on the correlation coefficients between FFA and target regions. A significant three-way interaction [FFA * context $*$ target region] was found $(F(3.055)=3.681 p<0.05)$. The [context $*$ target region] interaction was considered for left and right FFA separately, and was significant for both (left FFA: $F(4.898)=2.564 p<0.05$; right FFA: $F(3.221)=3.123 p<0.05)$.

We compared the connectivity between FFAs and target regions separately for PER and CAT condition, using paired-samples t-tests. After correction for multiple comparisons, the left precuneus showed a significantly stronger correlation with both FFAs (left: $\left(t(8)=3.196 p_{\text {corrected }}<0.05\right)$; right: $\left.\left(t(9)=3.265 p_{\text {corrected }}<0.05\right)\right)$ during the PER condition than during the CAT condition. Uncorrected, an identical correlation pattern was found between left FFA and right angular gyrus $\left(t(8)=1.930 p_{\text {uncorrected }}<0.05\right)$, and between right FFA and left IFG $\left(t(9)=2.419 p_{\text {uncorrected }}<0.05\right)$ and right angular gyrus $(t(9)=$ $\left.2.263 p_{\text {uncorrected }}<0.05\right)$. The two arbitrary control regions showed no difference between the conditions. Thus, connectivity between FFA and target regions overall was stronger when words were presented in a person-related context, but particularly so for left precuneus, and as trend for right angular gyrus. See figure 3.5 for an overview. 


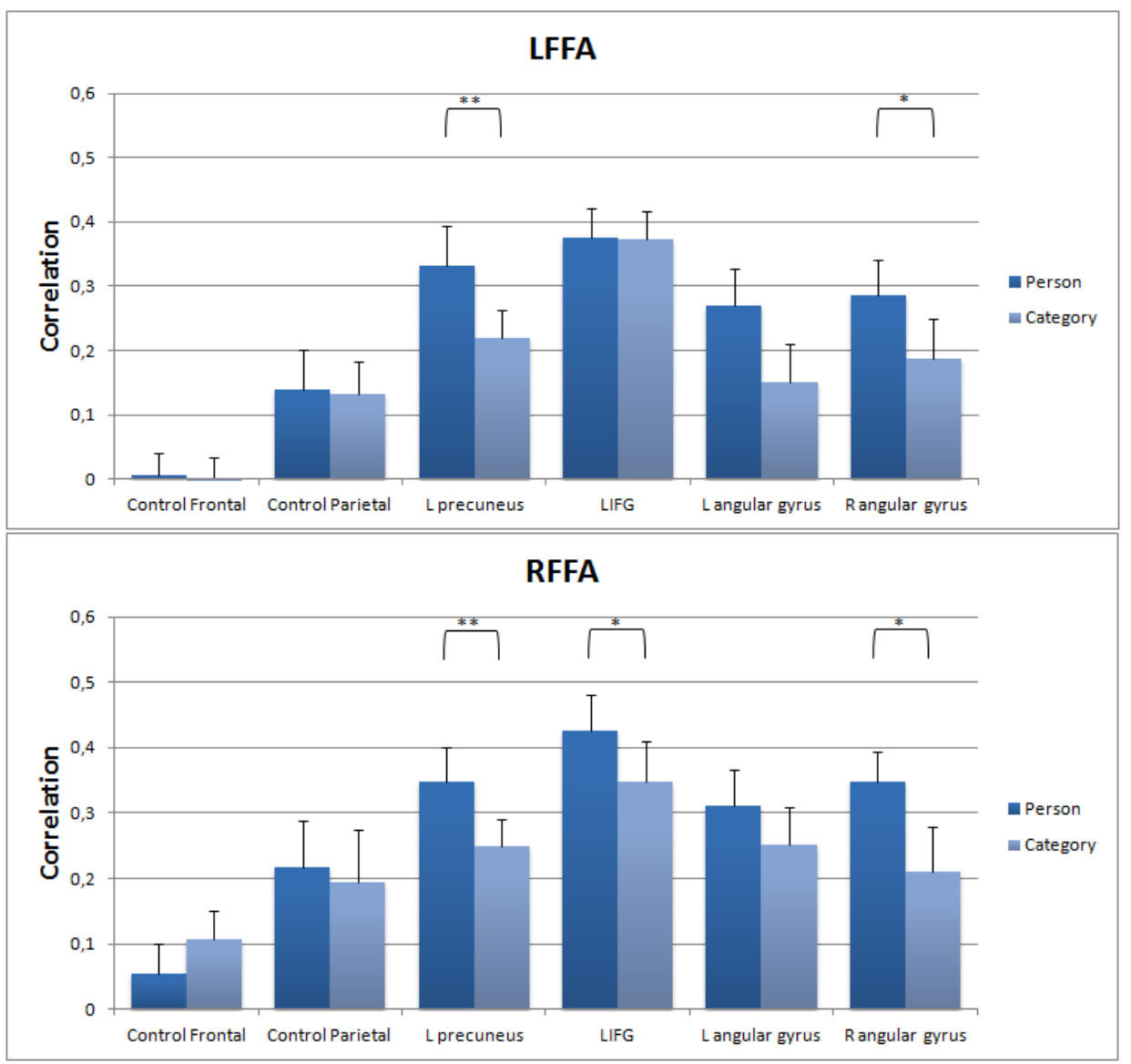

Figure 3.5. Pair-wise correlation coefficients. The top of the figure shows the correlation between the left Fusiform Face Area (LFFA) and the 4 target regions +2 controls. The bottom of the figure represents the correlation between right Fusiform Face Area (RFFA) and the target and control regions. Correlation coefficients are computed and presented separately for the person and category conditions. It can be seen that the BOLD signal of the left precuneus correlates significantly higher with both right and left FFA in the person condition than in the category condition. Moreover, without correcting for multiple comparisons, we found the correlation coefficients between left FFA and right angular gyrus to differ significantly between the person and category condition, in favor of the person condition. This same pattern was found between right FFA and left inferior frontal gyrus (LIFG), and between right FFA and right angular gyrus. Note that the histograms reflect the average correlation coefficients, but the error bars and significance levels are based on the coefficients after Fisher transformation, making them suitable for statistical comparison. See text for details. $* * p<0.05 * p<0.05$ (uncorrected) 
The inter-regional connectivity between the target regions was also investigated (see figure 3.6). The 6 pair wise correlation coefficients were computed for both contexts. The statistical significance was assessed with a repeated measures GLM with factors pair (6) and context (2). The interaction [pair * context] was not significant $(F(5)=0.225, p=0.936)$. Also the two main effects were not significant: context $(F(1)=0.763, p=0.405)$ and pair $(F(5)=$ $3.239, p=0.112)$. In despite of the results of the omnibus tests, we performed paired $t$-tests to adopt a more sensitive approach. Still, none of the pairs differed significantly between the person and category context. This means that the context bias affecting the coupling between regions only exists between FFA and some target regions, but not among the target regions themselves.

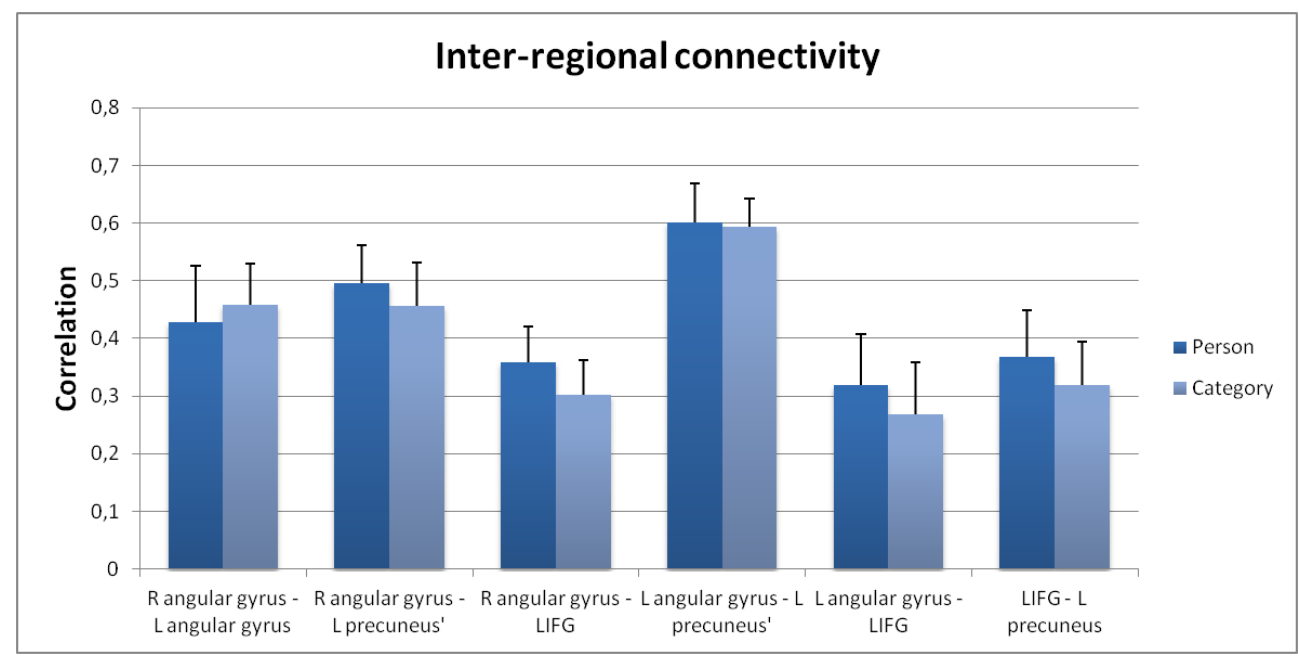

Figure 3.6. Inter-regional correlation coefficients. The connection strength between all region pairs (control regions and FFA excluded) did not differ between the person and category conditions. As in figure 3.4, note that the histograms reflect the average correlation coefficients, but the error bars and significance levels are based on the coefficients after Fisher transformation, making them suitable for statistical comparison. 


\subsection{Discussion}

In the current study, we investigated the possibility that FFA plays a role in a network involved in semantic - as opposed to visually based - person identification. Experimentally, we used identical stimuli across conditions, and we used words instead of faces, eliminating any visual context effects. We aimed to identify functional connections between FFA and other brain regions that are sensitive to a subtle person/category context manipulation within the presented blocks of words. We observed for relevant areas: the precuneus, left inferior frontal gyrus (LIFG) and bilateral angular gyri. If these regions indeed had a functional connection with FFA and these connections are used during the processing of person-related information, we expected this coupling to be stronger during the presentation of the person-related context than during the categorical context. Indeed, both with left and right FFA, we found that the precuneus has a significantly stronger functional relationship during the processing of the person context.

From these results, we conclude that there is a specific functional coupling between the precuneus and bilateral FFA, with a striking stronger coupling for the person context compared to the category context. Apparently, the person context engages a network of brain regions in which FFA takes part. In contrast, the category context does engage FFA to a much lesser degree, if not at all. Our earlier finding that FFA seems to differentiate between the person and categorical context (van den Hurk, et al., 2011; see Chapter 2) did not allow for any speculation about FFA 'preferring' the person context over the category context. The results from the current study, however, suggest a direct and specific involvement of FFA in the processing of conceptual identity information without direct face presentation. The potential alternative interpretation of the results, that the mere categorical variance in the person context (one exemplar of all categories) compared to the categorical context (all exemplars from one category) leads to adaptation in the category context, does not explain the specific involvement of FFA. Any general attention, working memory or adaptation effect would very likely have led to an increased coupling between all regions identified by the searchlight. We have demonstrated that this is not the case (see figure 3.5 ).

In order to make the person context easier to encode for our participants, we used a face image as instruction image prior to each block of words, while the category context was preceded by an image of an object. One could argue that the presentation of the face affects the activity in FFA during the 
context processing. In order to prevent direct spilling over, we used a 10 seconds inter-stimulus interval between the instruction cue and the consecutive block of words. Based on pilot experiments this interval was chosen such that the BOLDresponse of the cue returned to baseline prior to the presentation of the words (see figure 3.3). It is in contrast to previous studies that have shown delay-period activation in FFA when subjects were asked to maintain a face representation in working memory (Druzgal \& D'Esposito, 2001, 2003; Johnson, Mitchell, Raye, D'Esposito, \& Johnson, 2007; Postle, Druzgal, \& D'Esposito, 2003; Ranganath, DeGutis, \& D'Esposito, 2004). Moreover, it is most likely that any carryover effect from the face image, for instance during mental imagery, would elicit a stronger response in FFA during the PER condition than in the CAT condition (O'Craven \& Kanwisher, 2000). The absence of these two indicators in our data suggests that our observed effect was not caused by confounding influences from the instruction.

The searchlight identified, among other regions, the precuneus. Converging evidence exists that the precuneus is involved in processes related to memory of face and person identity. For instance, neuroimaging studies showed that this region prefers information related to face identity compared to face expression (Fox, Moon, Iaria, \& Barton, 2009). Moreover, the precuneus is more strongly activated when presented with familiar faces than unfamiliar faces (Gobbini \& Haxby, 2006), and is possibly involved in the retrieval of knowledge about a face (Gobbini \& Haxby, 2007; Gobbini, Leibenluft, Santiago, \& Haxby, 2004; Kosaka, et al., 2003). In addition, a recent study demonstrated that the precuneus may be part of a functional network involved in face integration (Kottlow, Jann, Dierks, \& Koenig, 2012), together with the face-sensitive middle fusiform gyrus. Von Kriegstein and colleagues (2005) assessed functional connectivity between the precuneus and FFA during a voice identification task and showed that the connection strength between the precuneus and FFA increases significantly after having associated the voices with faces (von Kriegstein \& Giraud, 2005). Still, this region has been shown to be involved in a wide variety of other cognitive processes such as consciousness, personality, and memory (Adelstein, et al., 2011; Cavanna, 2007; Cavanna \& Trimble, 2006; Sajonz, et al., 2010; Vogt \& Laureys, 2005). Because of this seeming heterogeneous functionality of the precuneus, one needs to be careful interpreting its apparently stronger coupling with FFA during the processing of the person-related condition.

A connectivity study by Margulies and colleagues demonstrated that the precuneus can be divided up into three subdivisions, each with distinct functionality and projections onto other cortical areas (Margulies, et al., 2009). A 
recent study extended these findings (Zhang \& Li, 2012) by employing a large group analysis of resting state fMRI data. Based on their results, the authors describe three subdivisions of the precuneus, referring to them as the dorsalanterior, dorsal-posterior and ventral precuneus. The location of the ventral precuneus as defined by Zhang and colleagues matches the location of the cluster identified by our searchlight and is positively connected to, among other regions, the parahippocampus and left and right angular gyrus (Zhang \& Li, 2012). The precuneus and bilateral angular gyri are known to be part of what is called the default mode network (DMN), a network of cortical regions that show pertinent activity during passive or rest conditions when compared to task-induced activity (for a review, see Buckner, Andrews-Hanna, \& Schacter, 2008). In a recent study, a dissociation of functions was observed in the DMN, with the precuneus and angular gyrus strongly involved in memory retrieval (Sestieri, Corbetta, Romani, \& Shulman, 2011; Vilberg \& Rugg, 2008). In both macaque (Rushworth, Behrens, \& Johansen-Berg, 2006) and human (Uddin, et al., 2010; Vincent, et al., 2006) cortex, the angular gyrus is anatomically and functionally connected to medial temporal structures such as hippocampal regions and the parahippocampal gyrus. In summary, these studies illustrate both at the level of anatomical connections and on the level of functionality the involvement of the precuneus and angular gyrus in memory and integration processes. 


\subsection{Conclusion}

Based on our findings and on previous work in the field, we believe that the precuneus and angular gyri are part of the network that facilitates memory processes such as the encoding and retrieval of the relevant semantic information. In the present study, the precuneus and angular gyri were identified by the multivariate searchlight analysis as treating the person context and the category context differently. The searchlight did not identify other memory related regions in such a way. Based on these results, we can assume that in the current study the precuneus and angular gyri are specifically involved in the integration of person related information in the person condition. This integration process is likely facilitated by neural activation within FFA, functioning as a hub sensitive to face identity (Andrews \& Ewbank, 2004; Barton, et al., 2002; Gauthier, et al., 2000; Grill-Spector, et al., 2004; Haxby, et al., 2002; Hoffman \& Haxby, 2000; Loffler, et al., 2005). This notion is strengthened by our finding of functionally specific coupling. Whereas the target regions themselves show no bias towards one of the two conditions in their inter-region correlation, the coupling characteristics between FFA and the other areas does. This coupling pattern implies it is solely the FFA that shows a stronger functional coupling with other regions - and this only during the processing of the semantic person related information. It also implies that it is not the person context itself that induces an overall gain increase in the target network where the category context does not. Overall, these findings suggest that FFA is specifically recruited during the association of semantic features in a person context. This, in turn, raises the possibility that neural processes involved in person identification might interact with FFA, even without face presentation. This notion is supported by studies which investigated auditory context effects in FFA. For example, it has been shown that the BOLD activity in FFA is modulated during the identification of familiar voices (von Kriegstein, Kleinschmidt, Sterzer, \& Giraud, 2005), likely via a structural connection between FFA and voice selective regions located on the upper bank of the superior temporal sulcus (Blank, Anwander, \& von Kriegstein, 2011). Importantly, our results go beyond the level of person identification based on visual or auditory percepts. They show that the processing of conceptual person identities might be supported by activation of information on a certain person (and his/her face) represented within FFA. This supportive recruitment of information apparently can be at work without actual perceptual input of face information. We could clearly show that FFA and its coupling with the relevant processing network can be activated via words, but only if those words associate with person-related information. FFA may hence facilitate the maintenance and/or retrieval of relevant biographic information of a 
person, and may be able to activate and or retrieve the relevant information. The present results have far reaching implications for the leading notion that FFA exclusively processes visual features. Future studies will have to investigate the exact relevance that FFA has in this process, as well as the underlying neural mechanisms. 


\subsection{Acknowledgements}

We are very grateful to Giancarlo Valente for helpful methodological insights, Tom de Graaf and Anne Havermans for comments on earlier versions of this manuscript and Michelle Moerel, Francesco Gentile, Milene Bonte and Joao Correia for useful discussions and comments. 


\subsection{References}

Adelstein, J. S., Shehzad, Z., Mennes, M., Deyoung, C. G., Zuo, X. N., Kelly, C., et al. (2011). Personality is reflected in the brain's intrinsic functional architecture. PLoS One, 6(11), e27633.

Andrews, T. J., \& Ewbank, M. P. (2004). Distinct representations for facial identity and changeable aspects of faces in the human temporal lobe. Neuroimage, 23(3), 905-913.

Barton, J. J., Press, D. Z., Keenan, J. P., \& O'Connor, M. (2002). Lesions of the fusiform face area impair perception of facial configuration in prosopagnosia. Neurology, 58(1), 71-78.

Bentin, S., Allison, T., Puce, A., Perez, E., \& McCarthy, G. (1996). Electrophysiological Studies of Face Perception in Humans. J Cogn Neurosci, 8(6), 551-565.

Bentin, S., \& Deouell, L. Y. (2000). Structural encoding and identification in face processing: erp evidence for separate mechanisms. Cogn Neuropsychol, 17(1), 35-55.

Blank, H., Anwander, A., \& von Kriegstein, K. (2011). Direct structural connections between voice- and face-recognition areas. J Neurosci, 31(36), 12906-12915.

Bruce, V., \& Young, A. (1986). Understanding face recognition. Br J Psychol, 77 ( Pt 3), 305-327.

Buckner, R. L., Andrews-Hanna, J. R., \& Schacter, D. L. (2008). The brain's default network: anatomy, function, and relevance to disease. Ann $N Y$ Acad Sci, 1124, 1-38.

Cavanna, A. E. (2007). The precuneus and consciousness. CNS Spectr, 12(7), 545-552.

Cavanna, A. E., \& Trimble, M. R. (2006). The precuneus: a review of its functional anatomy and behavioural correlates. Brain, 129(Pt 3), 564583. 
Druzgal, T. J., \& D'Esposito, M. (2001). Activity in fusiform face area modulated as a function of working memory load. Brain Res Cogn Brain Res, 10(3), 355-364.

Druzgal, T. J., \& D'Esposito, M. (2003). Dissecting contributions of prefrontal cortex and fusiform face area to face working memory. $J \operatorname{Cogn}$ Neurosci, 15(6), 771-784.

Eimer, M. (2000). Effects of face inversion on the structural encoding and recognition of faces. Evidence from event-related brain potentials. Brain Res Cogn Brain Res, 10(1-2), 145-158.

Fisher, R. A. (1915). Frequency distribution of the values of the correlation coefficient in samples of an indefinitely large population. Biometrika, 10(4), 507-521.

Fox, C. J., Moon, S. Y., Iaria, G., \& Barton, J. J. (2009). The correlates of subjective perception of identity and expression in the face network: an fMRI adaptation study. Neuroimage, 44(2), 569-580.

Friston, K. J., Buechel, C., Fink, G. R., Morris, J., Rolls, E., \& Dolan, R. J. (1997). Psychophysiological and modulatory interactions in neuroimaging. Neuroimage, 6(3), 218-229.

Gauthier, I., Tarr, M. J., Moylan, J., Skudlarski, P., Gore, J. C., \& Anderson, A. W. (2000). The fusiform "face area" is part of a network that processes faces at the individual level. J Cogn Neurosci, 12(3), 495-504.

Gobbini, M. I., \& Haxby, J. V. (2006). Neural response to the visual familiarity of faces. Brain Res Bull, 71(1-3), 76-82.

Gobbini, M. I., \& Haxby, J. V. (2007). Neural systems for recognition of familiar faces. Neuropsychologia, 45(1), 32-41.

Gobbini, M. I., Leibenluft, E., Santiago, N., \& Haxby, J. V. (2004). Social and emotional attachment in the neural representation of faces. Neuroimage, 22(4), 1628-1635.

Goebel, R., Esposito, F., \& Formisano, E. (2006). Analysis of functional image analysis contest (FIAC) data with brainvoyager QX: From singlesubject to cortically aligned group general linear model analysis and 
self-organizing group independent component analysis. Hum Brain Mapp, 27(5), 392-401.

Grill-Spector, K., Knouf, N., \& Kanwisher, N. (2004). The fusiform face area subserves face perception, not generic within-category identification. Nat Neurosci, 7(5), 555-562.

Haxby, J. V., Hoffman, E. A., \& Gobbini, M. I. (2002). Human neural systems for face recognition and social communication. Biol Psychiatry, 51(1), 59-67.

Henson, R. N., Goshen-Gottstein, Y., Ganel, T., Otten, L. J., Quayle, A., \& Rugg, M. D. (2003). Electrophysiological and haemodynamic correlates of face perception, recognition and priming. Cereb Cortex, 13(7), 793805 .

Hoffman, E. A., \& Haxby, J. V. (2000). Distinct representations of eye gaze and identity in the distributed human neural system for face perception. Nat Neurosci, 3(1), 80-84.

Johnson, M. R., Mitchell, K. J., Raye, C. L., D'Esposito, M., \& Johnson, M. K. (2007). A brief thought can modulate activity in extrastriate visual areas: Top-down effects of refreshing just-seen visual stimuli. Neuroimage, 37(1), 290-299.

Kanwisher, N., McDermott, J., \& Chun, M. M. (1997). The fusiform face area: a module in human extrastriate cortex specialized for face perception. $J$ Neurosci, 17(11), 4302-4311.

Kosaka, H., Omori, M., Iidaka, T., Murata, T., Shimoyama, T., Okada, T., et al. (2003). Neural substrates participating in acquisition of facial familiarity: an fMRI study. Neuroimage, 20(3), 1734-1742.

Kottlow, M., Jann, K., Dierks, T., \& Koenig, T. (2012). Increased phase synchronization during continuous face integration measured simultaneously with EEG and fMRI. Clin Neurophysiol.

Kriegeskorte, N., \& Bandettini, P. (2007). Analyzing for information, not activation, to exploit high-resolution fMRI. Neuroimage, 38(4), 649662. 
Kriegeskorte, N., Goebel, R., \& Bandettini, P. (2006). Information-based functional brain mapping. Proc Natl Acad Sci U S A, 103(10), 38633868 .

Loffler, G., Yourganov, G., Wilkinson, F., \& Wilson, H. R. (2005). fMRI evidence for the neural representation of faces. Nat Neurosci, 8(10), 1386-1390.

Ma, Y., \& Han, S. (2011). Functional dissociation of the left and right fusiform gyrus in self-face recognition. Hum Brain Mapp.

Margulies, D. S., Vincent, J. L., Kelly, C., Lohmann, G., Uddin, L. Q., Biswal, B. B., et al. (2009). Precuneus shares intrinsic functional architecture in humans and monkeys. Proc Natl Acad Sci U S A, 106(47), 2006920074 .

Mur, M., Ruff, D. A., Bodurka, J., Bandettini, P. A., \& Kriegeskorte, N. (2010). Face-identity change activation outside the face system: "release from adaptation" may not always indicate neuronal selectivity. Cereb Cortex, 20(9), 2027-2042.

O'Craven, K. M., \& Kanwisher, N. (2000). Mental imagery of faces and places activates corresponding stiimulus-specific brain regions. $J$ Cogn Neurosci, 12(6), 1013-1023.

Postle, B. R., Druzgal, T. J., \& D'Esposito, M. (2003). Seeking the neural substrates of visual working memory storage. Cortex, 39(4-5), 927-946.

Puce, A., Allison, T., Gore, J. C., \& McCarthy, G. (1995). Face-sensitive regions in human extrastriate cortex studied by functional MRI. J Neurophysiol, 74(3), 1192-1199.

Ranganath, C., DeGutis, J., \& D'Esposito, M. (2004). Category-specific modulation of inferior temporal activity during working memory encoding and maintenance. Brain Res Cogn Brain Res, 20(1), 37-45.

Rushworth, M. F., Behrens, T. E., \& Johansen-Berg, H. (2006). Connection patterns distinguish 3 regions of human parietal cortex. Cereb Cortex, 16(10), 1418-1430. 
Sajonz, B., Kahnt, T., Margulies, D. S., Park, S. Q., Wittmann, A., Stoy, M., et al. (2010). Delineating self-referential processing from episodic memory retrieval: common and dissociable networks. Neuroimage, 50(4), 1606-1617.

Sestieri, C., Corbetta, M., Romani, G. L., \& Shulman, G. L. (2011). Episodic memory retrieval, parietal cortex, and the default mode network: functional and topographic analyses. J Neurosci, 31(12), 4407-4420.

Uddin, L. Q., Supekar, K., Amin, H., Rykhlevskaia, E., Nguyen, D. A., Greicius, M. D., et al. (2010). Dissociable connectivity within human angular gyrus and intraparietal sulcus: evidence from functional and structural connectivity. Cereb Cortex, 20(11), 2636-2646.

van den Hurk, J., Gentile, F., \& Jansma, B. M. (2011). What's behind a face: person context coding in fusiform face area as revealed by multivoxel pattern analysis. Cereb Cortex, 21(12), 2893-2899.

Verosky, S. C., \& Turk-Browne, N. B. (2012). Representations of facial identity in the left hemisphere require right hemisphere processing. $J$ Cogn Neurosci, 24(4), 1006-1017.

Vilberg, K. L., \& Rugg, M. D. (2008). Memory retrieval and the parietal cortex: a review of evidence from a dual-process perspective. Neuropsychologia, 46(7), 1787-1799.

Vincent, J. L., Snyder, A. Z., Fox, M. D., Shannon, B. J., Andrews, J. R., Raichle, M. E., et al. (2006). Coherent spontaneous activity identifies a hippocampal-parietal memory network. J Neurophysiol, 96(6), 35173531.

Vogt, B. A., \& Laureys, S. (2005). Posterior cingulate, precuneal and retrosplenial cortices: cytology and components of the neural network correlates of consciousness. Prog Brain Res, 150, 205-217.

von Kriegstein, K., \& Giraud, A. L. (2006). Implicit multisensory associations influence voice recognition. PLoS Biol, 4(10), e326.

von Kriegstein, K., Kleinschmidt, A., Sterzer, P., \& Giraud, A. L. (2005). Interaction of face and voice areas during speaker recognition. $J$ Cogn Neurosci, 17(3), 367-376. 
FFA - Precuneus Interaction During Processing of Words in Biographic Context

Zhang, S., \& Li, C. S. (2012). Functional connectivity mapping of the human precuneus by resting state fMRI. Neuroimage, 59(4), 3548-3562. 



\section{Chapter 4}

\section{Functional Segregation of Mid- and Posterior 'Fusiform Face Area'}

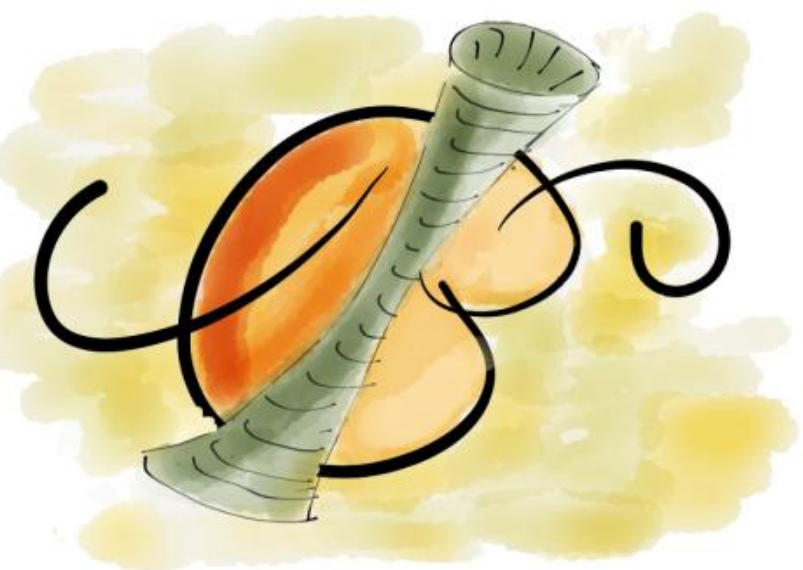

${ }^{3}$ Van den Hurk, J., Goebel R., \& Jansma B.M. Functional Segregation of Midand Posterior 'Fusiform Face Area'. Submitted. 


\section{Abstract}

The involvement of the Fusiform Face Area in face processing is well established, but its exact functional contribution remains an important topic of research. A recent anatomical subdivision of FFA into two clusters asks for a reinvestigation of the underlying functional specialization of these subdivisions. In this fMRI study, we repeatedly presented subjects with 3 distinct identities (a face followed by words describing the individual). In addition, we carefully localized face sensitive clusters on fusiform cortex using an independent localizer. We were able to localize two distinct regions within traditional boundaries of FFA: the first located in the middle portion of the fusiform sulcus, the second positioned more laterally on the posterior fusiform gyrus. We then used multi-voxel pattern analysis to investigate whether we could decode identity information from activity patterns in these clusters, thereby making a distinction between activity elicited by the face and activity evoked by the semantic description of the identity. We found a functional double dissociation between two distinct clusters within FFA in their contribution to visual and semantic identity processing. 


\subsection{Introduction}

When we are introduced to someone new, biographic information we learn about him or her is integrated with the observed facial features. After consolidation, this biographic information is seamlessly retrieved as soon as we see the face again, and as soon as detailed semantic information is provided, one is easily able to visualize the accompanying face. For years, the involvement of the Fusiform Face Area (FFA) in the recognition of faces has been studied (Kanwisher, McDermott, \& Chun, 1997; Kanwisher \& Yovel, 2006), but the conclusions regarding the involvement in identity processing have been inconclusive. Evidence exists that FFA is sensitive to face identity (Andrews \& Ewbank, 2004; Barton, Press, Keenan, \& O'Connor, 2002; Gauthier, et al., 2000; Grill-Spector, Knouf, \& Kanwisher, 2004; Haxby, Hoffman, \& Gobbini, 2002; Hoffman \& Haxby, 2000; Loffler, Yourganov, Wilkinson, \& Wilson, 2005), and even voice (von Kriegstein, Kleinschmidt, Sterzer, \& Giraud, 2005) and semantic identity information (van den Hurk, et al., 2011; van den Hurk, et al., submitted; see Chapter 2 and Chapter 3), but so far no study was able to decode distinct identity representations from spatial patterns of neural activity in FFA (Kriegeskorte, Formisano, Sorger, \& Goebel, 2007; Mur, Ruff, Bodurka, Bandettini, \& Kriegeskorte, 2010; but see Nestor, Plaut, \& Behrmann, 2011).

Differences in stimulation paradigms and analysis techniques among these studies, to some extent, explain differences in the interpretation of FFA and its role in identity processing. In addition, new insights in the anatomical division of FFA opens new avenues to study identity process within FFA in more detail. Very recently, it has been pointed out that typical procedures to localize FFA using fMRI result in several face-selective clusters across ventral temporal cortex, with two distinct regions within the fusiform gyrus (Pinsk, et al., 2009; Weiner \& Grill-Spector, 2010, 2011). In the past, these regions were commonly combined during the functional localization of FFA, often due to methodological limitations such as a coarse spatial resolution and spatial smoothing. However, anatomically adjacent regions responding to a single localizer task may nonetheless reveal fine-grained differential functionality. Merging multiple anatomically distinct regions into one may lead to a loss of fine-grained spatial information that can be decoded from these regions. With use of high-resolution fMRI and by paying close attention to anatomical landmarks, FFA now can be redefined into a mid-fusiform and a posterior-fusiform cluster; the former overlapping the mid-fusiform sulcus, and the latter located on the posterior fusiform gyrus (Weiner \& Grill-Spector, 2012). Could it be that anatomically 
segregating FFA into sub-regions provides a gain in the amount of information about identity that can be decoded from these regions?

By combining high-resolution fMRI (voxel size: $2 \times 2 \times 2 \mathrm{~mm}$ ) and a classification method based on a machine learning algorithm (linear support vector machine) (Cortes \& Vapnik, 1995; Formisano, De Martino, \& Valente, 2008), we investigated if we could disentangle the contribution of the midfusiform ( $m F u s)$ and posterior fusiform face area $(p F u s)$ to the processing of person identity. We presented subjects with 3 distinct identities while their brain activity was measured using fMRI. Each identity was presented as follows: first, a face was shown for 2 seconds, followed by 12 seconds of fixation. Then, a series of 5 keywords was presented that described the (semantic) identity of the face. Subjects were instructed to associate the semantic identity with the face. The rationale behind this approach is that during the face presentation, the visual (face) information is fed to the fusiform face clusters via early visual areas in a bottom-up way. After a few trials, the associated semantic information is automatically recalled (Bruce \& Young, 1986; Craigie \& Hanley, 1993, 1997) and is likely fed into fusiform face regions via top-down connections (Kriegeskorte, et al., 2007; van den Hurk, et al., submitted; see Chapter 3). Vice versa, when the semantic identity is presented to the subject, we hypothesize that the recalled face information is activated and fed to the fusiform face clusters. Our analysis therefore focuses on decoding distinct visual and semantic identity information from underlying patterns of activity in the mFus and pFus clusters. 


\subsection{Materials and methods}

\subsubsection{Participants}

Ten (mean age $=24,6$ females) healthy Dutch speaking subjects gave their written informed consent and participated in the study. None of the participants had a history of neurological abnormalities. Approval for the study was granted by the Ethical Committee of the Faculty of Psychology and Neuroscience at the University of Maastricht.

\subsubsection{Stimuli}

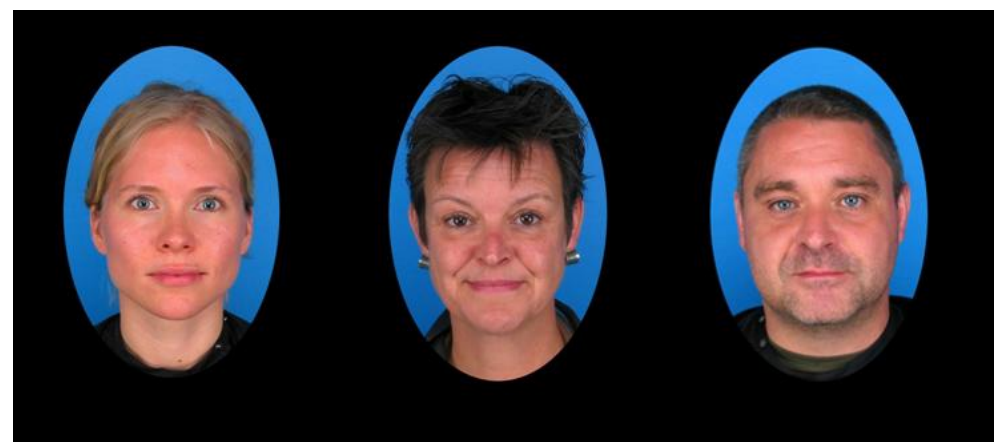

\begin{tabular}{l|lllll} 
Identity \# & Profession & Nationality & $\begin{array}{l}\text { Marital } \\
\text { status }\end{array}$ & Music style & Hobby \\
1 & Surgeon & Belgian & Married & Rock & Chess \\
2 & Conductor & German & Divorced & Classical & Soccer \\
3 & Teacher & French & Widowed & Jazz & Cooking
\end{tabular}

Figure 4.1. Faces (top) and words (bottom) used in experiment. The words in this table are translated from Dutch, as the actual stimuli were in Dutch. 


\subsubsection{Procedure}

While in the scanner, participants were presented with 4 experimental runs, each of approximately 10 minutes duration. An experimental run commenced with 20 seconds of fixation, after which the first identity trial was presented. At trial onset, the participants saw a face presented for 2 seconds followed by a 12 second inter stimulus interval to ensure that there is enough time for the hemodynamic response elicited by the face image to reach baseline level. Then, a block of 5 words was presented, each word lasting $1800 \mathrm{~ms}$, followed by $200 \mathrm{~ms}$ fixation. The word sequence was followed by 10 seconds of fixation again before a new face was presented. The 3 identity trials were presented in random order, and the sequence of the 3 identities was repeated 6 times per run, resulting in 24 presentations per identity after the experimental session. The participants were instructed to actively associate the words with the preceding face.

The sequence of five words assigned to a face was kept constant for a participant, i.e. each face was to be associated with the same semantic identity throughout the experiment (see figure 4.1). Across participants, however, the words were scrambled per category, resulting in a new combination of face and semantic identity per participant to avoid systematic category effects in memory associations.

During a separate run to localize face sensitive regions in the brain, the participant passively watched blocks of faces, scrambled faces and houses. A block consisted of 10 gray scale images, each presented $1500 \mathrm{~ms}$ followed by $300 \mathrm{~ms}$ fixation. Blocks were repeated 8 times and were presented in a random order to prevent expectation and adaptation effects.

\subsubsection{Data acquisition}

Functional and anatomical images were collected using a 3-T Siemens Magnetom Allegra head scanner (Siemens Medical System, Erlangen, Germany) using a standard head coil. Twenty-eight T2*-weighted echo-planar imaging (EPI) slices (voxel size $=2 \times 2 \times 2 \mathrm{~mm}$, inter slice gap $=0 \mathrm{~mm}$ ) covering the temporal lobe were recorded (repetition time $[\mathrm{TR}]=2000 \mathrm{~ms}$, matrix size $=128 \mathrm{x}$ 128 , echo time $[\mathrm{TE}]=30 \mathrm{~ms}$ ). For the localization of the face-sensitive clusters, twenty-five EPI slices with identical resolution were measured $([T R]=1800 \mathrm{~ms}$, matrix size $=128 \times 128,[\mathrm{TE}]=30 \mathrm{~ms})$. In addition, a high resolution $(1 \times 1 \times 1$ $\mathrm{mm}$ ) T1-weighted anatomical scan was obtained using an ADNI sequence ([TR] $=2250 \mathrm{~ms} ;[\mathrm{TE}]=2.6 \mathrm{~ms}$; flip angle $=9^{\circ}$ ). 
The participants were placed comfortably in the scanner and their head was fixed with soft foam pads. Participants saw the stimuli projected on a screen through a mirror mounted on the head coil. Stimulus presentation was synchronized with MR data acquisition by triggering the stimulus program (Presentation Software, Neurobehavioral Systems Inc, Albany, CA) with the first MR pulse.

\subsubsection{Analysis}

Prior to analysis, functional images were preprocessed using BrainVoyager QX 2.2 (Brain Innovation, Maastricht, The Netherlands). As a first step, the first 2 volumes were excluded due to T1-saturation. Preprocessing steps consisted of slice scan-time correction (using cubic spline interpolation), 3-dimensional subject motion correction (trilinear interpolation and sinc interpolation to detect and correct for motion, respectively) using 3 translation and 3 rotation parameters, and a high-pass temporal filter (including linear trend removal).

In order to localize face selective clusters in the ventral temporal cortex, a general linear model (GLM) was run on the time series. For each participant, a design matrix was created using a predictor for each of the three conditions (faces, houses, scrambled faces). To account for the hemodynamic response delay, the predicted time courses were convolved with a canonical (double gamma) hemodynamic response function (HRF). Subsequently, a conjunction analysis between [faces $>$ houses] and [faces $>$ scrambled faces] was considered (False Discovery Rate (FDR) corrected at $q<0.05$ ). In case not all 4 target clusters (mFus and pFus in both hemispheres) appeared at this threshold, the threshold was lowered until all regions were visible, provided that the condition $[p<0.05$, uncorrected for multiple comparisons] remained satisfied.

The first step of the MVP-analysis entailed the creation of the stimulusevoked hemodynamic response patterns. We used BrainVoyager QX 2.2 for trial-response estimation. Each pre-processed time series was first split into individual trials. This resulted in 24 separate trials for each of the 3 face trials, and for each of the 3 semantics trials blocks. Per trial, a multi-voxel pattern was generated for each of the previously defined fusiform face clusters: for each voxel belonging to a cluster, the individual response to a trial was estimated by fitting a general linear model and using the resulting regressor-coefficient (beta) as a representation of the voxel response amplitude. After this trial estimation procedure, the voxel response estimates were combined into a single spatial response pattern corresponding to the trial and fusiform cluster. 


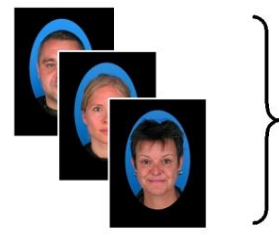

A.

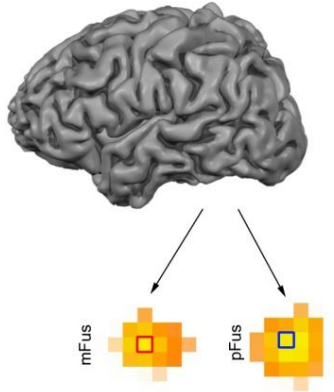

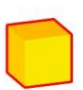

B.
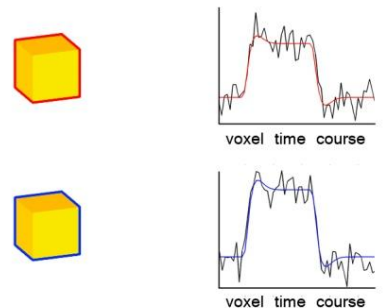

Figure 4.2. Feature extraction procedure, exemplified for face trials in this figure. A. Each face trial is presented 24 times while hemodynamic brain activity is measured. The $m F u s$ and pFus voxels selected during the analysis of the independent localizer run are subsequently used to create response patterns. B. Per voxel, an individual double-gamma hemodynamic response function (HRF) is fitted to each trial response to attain an estimate of the voxel response. After trial estimation, the voxel responses to a trial are combined into a response pattern for each fusiform cluster separately. In this manner, 24 response patterns are created per cluster for each face stimulus. This procedure was repeated for the semantics trials.

The training of the classification algorithm and consequent prediction computations were performed using MATLAB R2010b (Mathworks Inc, Natick, MA) and a linear support vector machine (SVM) (Formisano, et al., 2008; Mourao-Miranda, Bokde, Born, Hampel, \& Stetter, 2005; Cortes, et al., 1995), based on the LIBSVM algorithm (http://www.csie.ntu.edu.tw/ cjlin/libsvm/). Response patterns were first labelled with their corresponding condition label, e.g. 'face 1', 'face 2', 'face 3' and 'semantics 1', 'semantics 2', 'semantics 3'. The consequent analysis was performed for the face trials and semantics trials separately. For all 3 identities, the trial-based patterns were divided in into a training (21 trials) and a test ( 3 trials) subset. The labelled training trials were fed to the algorithm, which defines a multidimensional hyperspace. Each trial, or example, is expressed as a vector of $N$ features in this hyperspace. The features are represented by the voxels identified by the localizer, such that all 63 training examples (belonging to 3 different classes) are represented as points in the multidimensional space. It is important to note that in our analysis, 4 separate feature spaces are devised that represent the 4 fusiform face clusters. During the training phase, the SVM algorithm defines an optimal way of separating the 3 classes in the feature space. Subsequently, the unlabeled test examples that were initially left out of the training phase are now mapped in the same multidimensional space, and predicted to belong to one of the 3 classes based on 
the class-separation parameters of the trained model. The percentage of examples that could be correctly classified reflects the prediction accuracy of the model. The training-testing procedure is repeated in a cross-validation sequence $\left(N_{\text {folds }}=\right.$ 1000) with different training-testing subsets, in order to acquire a stable estimate of the average prediction accuracy.

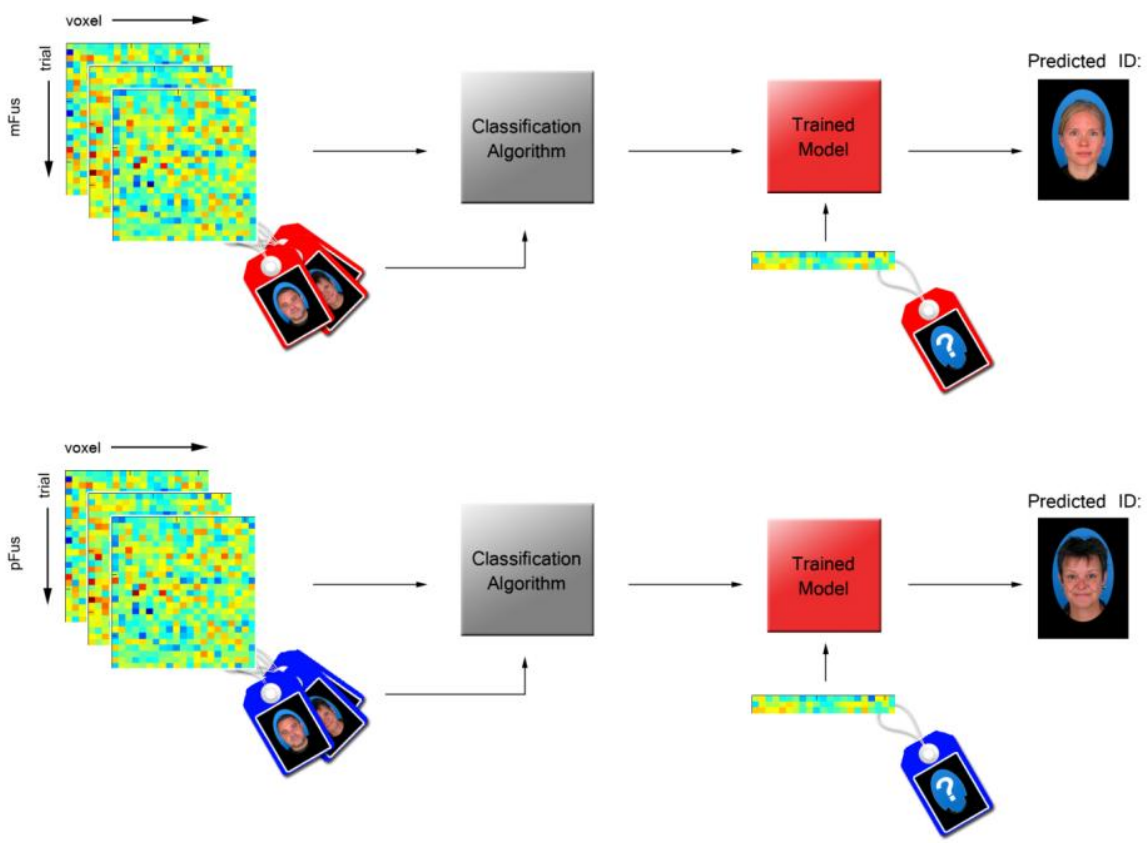

Figure 4.3. Classification procedure, exemplified for the face trials in this figure. Training and testing of the classification algorithm was performed for mid-fusiform (top stream) and posterior fusiform (bottom stream) clusters separately. From left to right: response patterns ( $k$ trials $x n$ voxels) are labelled with the corresponding face number. Data is split into a train- and test subset, after which the training patterns and corresponding class labels are fed into the classification algorithm (support vector machine). The classifier then modelled the relationship between the 3 faces and underlying hemodynamic response patterns. Subsequently, the unlabeled test trials were mapped onto the trained model in order to predict the corresponding face.

The MPVA procedure described above was repeated for each participant, for all 4 face sensitive regions per subject and for the semantics and faces separately, resulting in 10 particiants $\times 4$ clusters $\times 2$ conditions $=80$ 
repetitions. For all levels of our design, we performed a permutation test to empirically estimate the null hypothesis of our tests. For this permutation test, the splitting of the data into train- and test subset was identical to the previous analysis, but this time the labels were randomly assigned to the training trials. The classification algorithm then 'learns' the arbitrary relationship between the response patterns and randomly assigned labels, after which the prediction accuracy is assessed using the unused test trials. These steps were also repeated using a cross-validation approach $\left(N_{\text {folds }}=1000\right)$, resulting in an average permutation accuracy per participant $\mathrm{x}$ fusiform cluster $\mathrm{x}$ condition.

The computed accuracy parameters per subject originate from a multinomial (categorical) distribution. Therefore, since the normality assumption was not met, a non-parametric approach was used for assessing statistical significance. In order to test the variables for possible interaction effects, we computed the 3-way $F$-statistic using a repeated-measures ANOVA with the factors left/right hemisphere (2) x mid/posterior fusiform (2) x faces/semantics (2). Then, we permuted the $3 \times 2$ condition labels across participants and recomputed the $F$-statistic in 10,000 iterations, creating the distribution of the $F$ under the null hypothesis (Suckling \& Bullmore, 2004). The probability of finding the true interaction $F$-statistic under the assumption that the $\mathrm{H}_{0}$ is true was subsequently derived from the permuted $F$ distribution. This process was repeated for the 2-way interaction.

To test whether individual regions predicted the semantics and faces significantly better than their permutation counterparts, a non-parametric statistical comparison between the predicted and permuted sample was computed using a Wilcoxon signed-rank test. 


\subsection{Results}

In this study, we first localized mFus and pFus bilaterally in each participant using an independent functional localizer run (see section 4.3.1). Then, for the faces- and semantics trials individually, we trained a classification algorithm with a subset of labelled patterns from all 3 identities, after which we assessed the accuracy at which our algorithm could classify the remaining unlabeled patterns (see Supplementary Fig. 3). By means of cross-validation approach, the average prediction accuracy was estimated. These steps were repeated for all 4 fusiform regions (see Supplementary Methods).

\subsubsection{Localization of face sensitive clusters}

The results of the face sensitive localizer are presented for a representative subject in figure 4.4. For clarity, the results are projected on a flattened representation of the reconstructed cortex along the white matter / grey matter boundary of the subject's brain. As can be seen at the spatial resolution of $2 \times 2 \times$ $2 \mathrm{~mm}$ and without spatial smoothing, two distinct clusters appear on the fusiform sulcus and lateral fusiform gyrus. After application of a Gaussian spatial filter, the clusters blend in to one region that is commonly referred to as the FFA. 


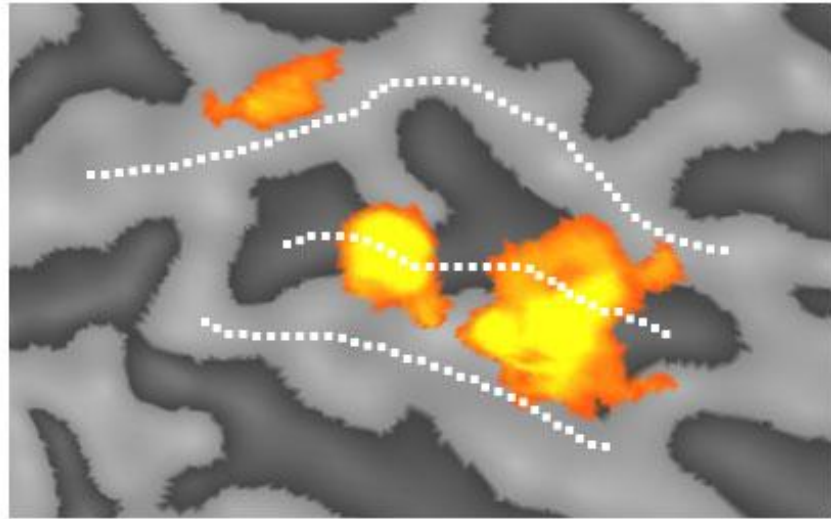

no smoothing

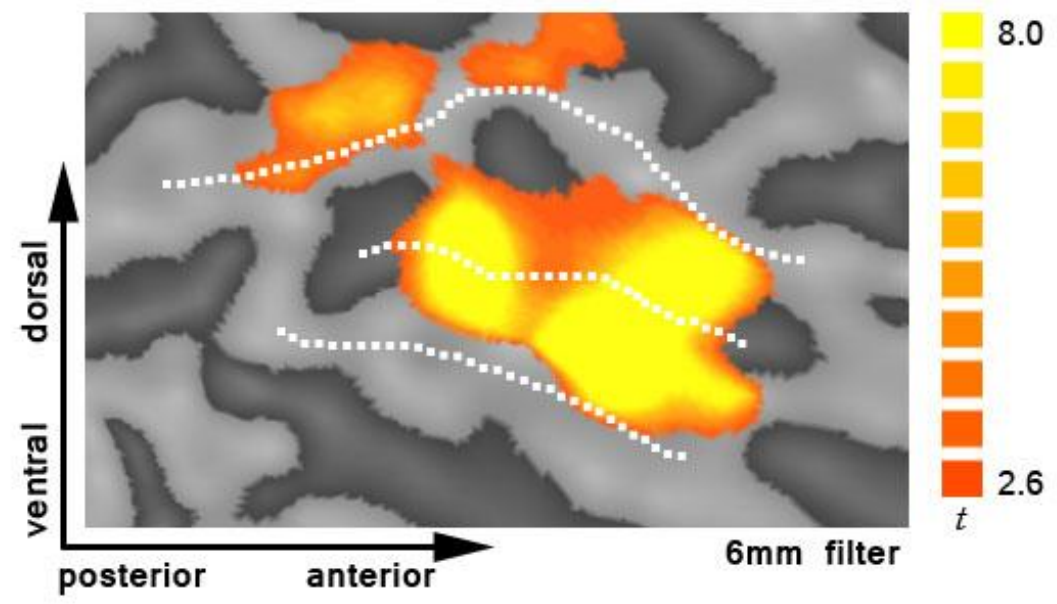

Figure 4.4. Face sensitive regions on ventral temporal cortex, unsmoothed (top) and after spatial smoothing (bottom). Without spatial smoothing (top), two distinct clusters can be distinguished along the middle fusiform sulcus (middle dashed line) and the middle lateral fusiform gyrus (bottom dashed line). After application of a Gaussian filter (FWHM $=6 \mathrm{~mm}$ ), the two regions blend into one cluster, classically referred to as the Fusiform Face Area (FFA). Occipital face area (OFA) can be identified on the inferior occipital gyrus (top dashed line). The contrast presented here is a conjunction of [Faces > Houses] and [Faces > Scrambled Faces], corrected for multiple comparisons using False Discovery Rate (FDR) $q=0.05$. 


\subsubsection{Analysis of prediction accuracies}

Because of the multinomial nature of the dependent variable, interaction effects were tested for significance by means of a permutation of the $F$-test (see section 4.2.5). The [left-right $*$ mFus-pFus $*$ faces-semantics] interaction was not significant $(F=0.002, p>0.9)$. We therefore collapsed the left and right hemispheres and focused on a possible difference in ID-processing between the mid and posterior cluster. Indeed, the [mFus-pFus * faces-semantics] interaction was significant $(F=18.6468, p<0.01)$, see figure 4.5 .

The resulting prediction accuracies, averaged across subjects, were then compared with the empirically estimated means of the null hypothesis derived from the permutation tests. We hypothesized a difference in information content between the mid-fusiform and posterior fusiform face regions. Indeed, for the mid-fusiform clusters, the faces trials could be predicted significantly above (permutation) chance level $(37.4 \%, \mathrm{p}<0.01)$, while the semantics trials could not (33.0\%, $\mathrm{p}>0.4$ ). The posterior-fusiform clusters showed the opposite pattern, with the semantics yielding an accuracy significantly higher than chance $(36.9 \%$, $\mathrm{p}<0.01)$ and the faces at chance level $(33.4 \%, \mathrm{p}>0.9)$, see figure 4.5 . The tests were performed with a Wilcoxon signed-rank test. 


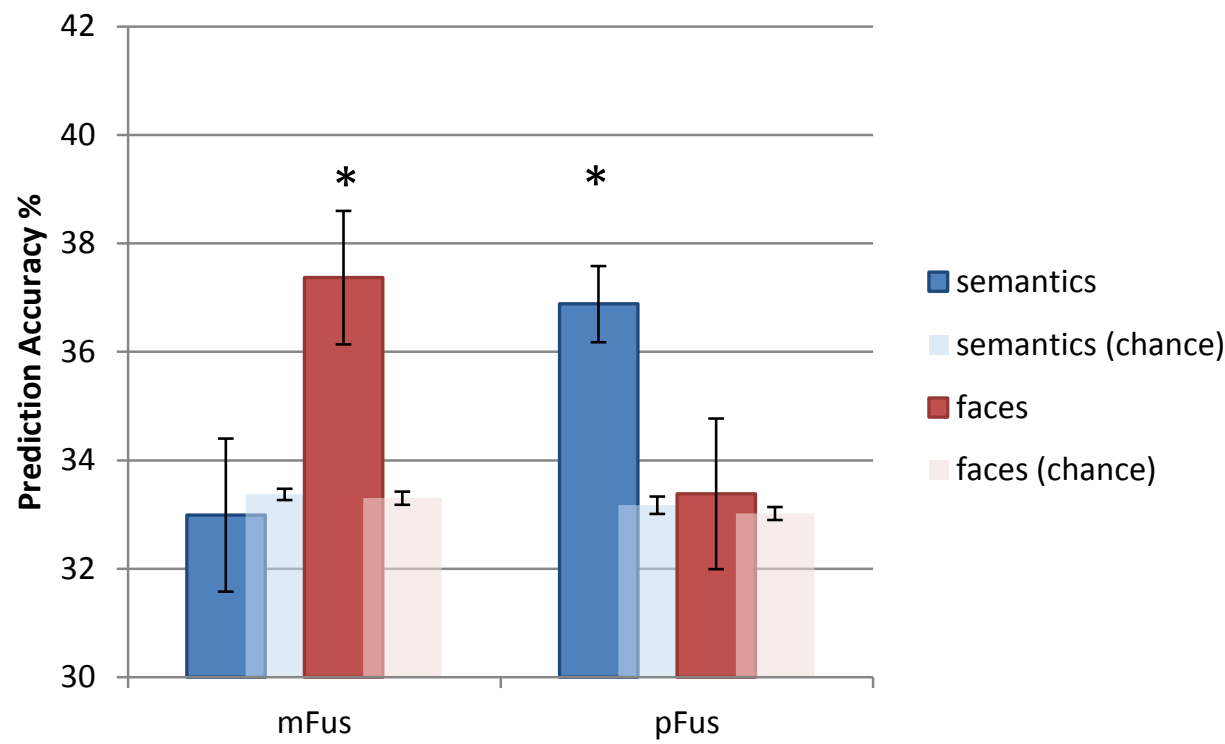

Figure 4.5. Prediction accuracies for the semantics (blue) and face (red) trials, compared to their permuted counterparts (transparent), splitted over mid (mFus) and posterior (pFus) fusiform face regions. * $p<0.01$

To prevent the information of the face from directly carrying over to the block of words, thereby contaminating the semantic identity information, we assured that the inter-stimulus interval between the face and the words was long enough for the fMRI-signal to go back to baseline. Still, we investigated whether the signal at the onset of the block of words contained information about the identities. We extracted the raw voxel values from the volume that corresponded to the onset of the block of words, and these response patterns were subsequently analyzed following the same classification procedure. We tested if the average classification accuracy across participants was higher than the accuracies derived from the permutation test and found that this was not the case for any of the fusiform clusters (all $p$-values within the range $0.99>p>0.7$ ). 
To investigate whether separating FFA into two sub clusters resulted in improved accuracies at which the classes could be predicted, the classification procedure was repeated with the mid-and posterior clusters grouped into left and right 'traditional' FFA. Neither the faces nor the semantics could be classified above permutation chance level in left FFA, while the right FFA allowed for above-chance decoding of the semantics $(38,1 \%, \mathrm{p}<0.01)$ but not the faces, see figure 4.6.

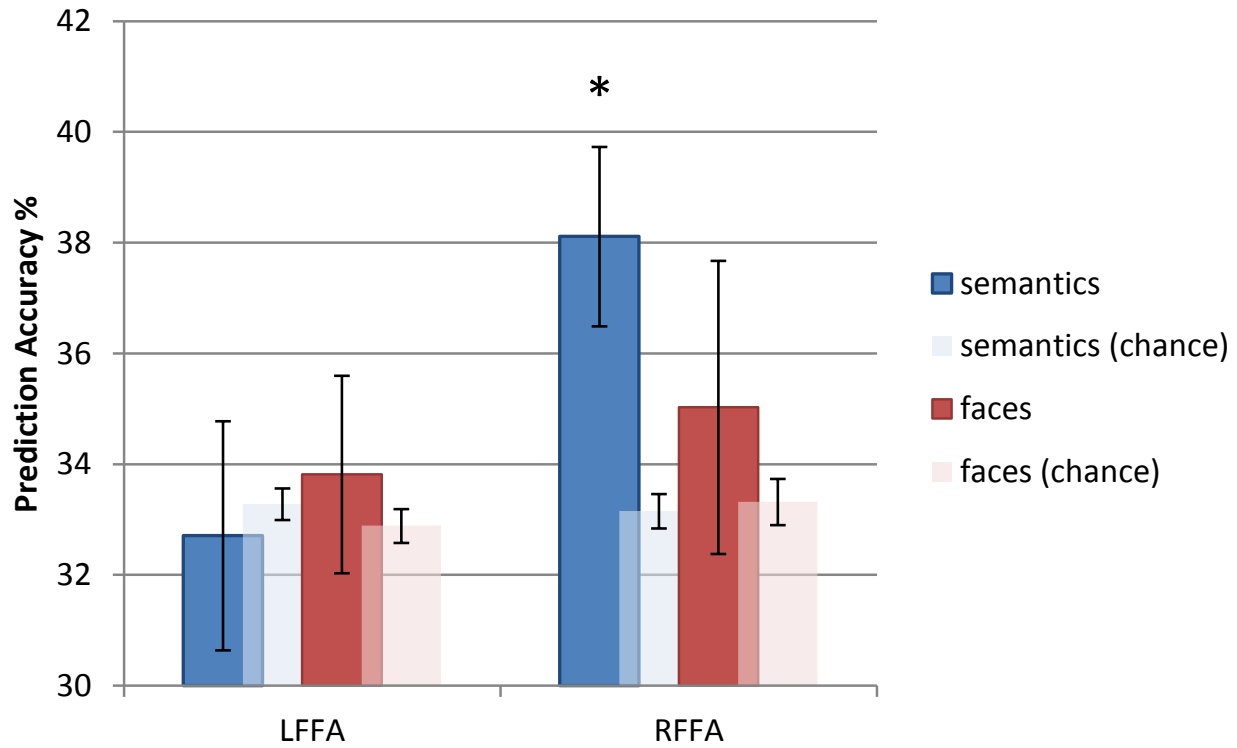

Figure 4.6. Prediction accuracies after collapsing the mid-and posterior fusiform clusters for left (LFFA) and right (RFFA) FFA. * $p<0.01$ 


\subsection{Discussion}

Our results show a functional double dissociation between mid- and posterior clusters within FFA, with distinct contributions to visual and semantic identity processing during the applied association task. During our experiment, whenever the participants see the face and mentally associate it with underlying semantic information (biographical background), prediction accuracy in mFus is well above chance, which is not the case for pFus. In contrast, when they see the words and mentally associate these with the related face, the prediction accuracy is reversed for pFus and mFus respectively. Notably, after combining the midand posterior clusters into one 'Fusiform Face Area' according to classic definitions, we witnessed a loss in the ability to decode the individual face trials from both left and right FFA. The semantics trials, however, could still be derived from response patterns in right FFA. In line with recent topographical evidence (Weiner \& Grill-Spector, 2010, 2011, 2012), these results point out that the traditional modular description of FFA may require a functional redefinition.

The process of associating faces with semantic knowledge as well as the automatic retrieval of this information is either based on local information retrieval or based on an interplay between bottom up and top down streams of information feeding into pFus and mFus. With the current design and results we cannot disentangle between local or bottom-up and top-down streams, hence we cannot conclusively conclude what type of information is picked up from the fusiform regions by our MVPA algorithm. Still, we know from the convergence perspective in vision that neurons in the visual ventral stream show an increase of receptive field size (Bles, Schwarzbach, De Weerd, Goebel, \& Jansma, 2006; Kastner, De Weerd, Desimone, \& Ungerleider, 1998; Kastner et al., 2001; Kastner, Pinsk, De Weerd, Desimone, \& Ungerleider, 1999). They also increase in the complexity of their tuning from V1 to aIT (Martin \& Chao, 2001). From that point of view, we argue that the information we decode from the mid fusiform region reflects a relatively complex identity representation that is available in that region during the presentation of a face. How does this information become available? Apparently, when the participant sees one of the faces, the corresponding semantic information is retrieved either directly from the mid fusiform area (local knowledge representation), or is sent to the mid fusiform region from other semantic processing regions. Vice versa, when the series of words is presented to the subject, visual aspects of the corresponding face is automatically retrieved either directly from the posterior fusiform area, or indirectly from memory and fed to the posterior fusiform cluster. This is in line with accounts of participants who reported to experience an automatic retrieval 
of the face when being presented with the written description of the corresponding person. They also reported an immediate retrieval of semantic information as soon as one of the faces was shown. Future studies may be able to disentangle the bottom-up stimulus based representations and the top-down information that is fed back from memory, by combining fMRI at ultra high magnetic fields with advanced analysis tools that can sample information from individual cortical layers in visual regions (Goebel, 2012; Zimmermann, et al., 2011). By focussing on the mid-cortical layer receiving bottom-up input, the specific information that is fed into the different fusiform face regions could eventually be examined.

Apparent functional modules such as FFA likely consist of a network of sub-regional clusters that lie beyond the spatial resolution of the imaging methods used. These clusters may in turn serve a purpose in a larger scale map coding for a particular feature dimension (Op de Beeck, Haushofer, \& Kanwisher, 2008). In order to resolve these questions, high resolution functional imaging at (sub-)millimetre range is required to carefully define regional boundaries. Subsequently, pattern recognition techniques can reveal the specific information content of these regions, thereby bringing us closer to unravelling the exact functional organization of ventral temporal cortex. 


\subsection{Conclusion}

Since the recent dissection of FFA into two adjacent, but distinct, functional sub clusters, the question arises whether the information content of these regions differs with respect to subtle aspects of face processing. In this study, we localized these two clusters (per hemisphere) in 10 participants. While measuring the activity in these clusters using fMRI, we presented the participants with the faces and subsequent semantic descriptions of three fictive individuals. Whenever participants saw a face, they actively associate background meaning to this face. Moreover, when they were presented with words conveying semantic information, they actively associated a corresponding face. Using a pattern recognition analysis technique, we showed that we could predict above chance level what face was presented at a particular trial based on activity in the mid-fusiform cluster alone, which may indeed relate to active retrieval of background information. Moreover, we could decode above chance level what semantic description was presented to the participants by looking at the patterns of activity in the posterior fusiform cluster, which may be related to active retrieval of facial information. Interestingly, we noticed a significant drop in the prediction accuracy when we collapsed the mid- and posterior clusters into FFA in traditional terms. Taken together, our observations strongly suggest not only an anatomical, but also a functional dissociation between the mid- and posterior fusiform face cluster during the processing of person identity. 


\subsection{Acknowledgements}

The authors are very grateful to Giancarlo Valente, Tom de Graaf, Michelle Moerel, Hans Op de Beeck, Martin Frost, Joao Correia, Francesco Gentile, Federico Demartino and Milene Bonte for helpful discussions and suggestions. 


\subsection{References}

Andrews, T. J., \& Ewbank, M. P. (2004). Distinct representations for facial identity and changeable aspects of faces in the human temporal lobe. Neuroimage, 23(3), 905-913.

Barton, J. J., Press, D. Z., Keenan, J. P., \& O'Connor, M. (2002). Lesions of the fusiform face area impair perception of facial configuration in prosopagnosia. Neurology, 58(1), 71-78.

Bles, M., Schwarzbach, J., De Weerd, P., Goebel, R., \& Jansma, B. M. (2006). Receptive field size-dependent attention effects in simultaneously presented stimulus displays. Neuroimage, 30(2), 506-511.

Bruce, V., \& Young, A. (1986). Understanding face recognition. Br J Psychol, 77 ( Pt 3), 305-327.

Cortes, C., \& Vapnik, V. (1995). Support-Vector Networks. Machine Learning, 20, 273-297.

Craigie, M., \& Hanley, J. R. (1993). Access to visual information from a name is contingent on access to identity-specific semantic information. Memory, 1(4), 367-391.

Craigie, M., \& Hanley, J. R. (1997). Putting faces to names. Br J Psychol, 88 ( Pt 1), 157-171.

Formisano, E., De Martino, F., \& Valente, G. (2008). Multivariate analysis of fMRI time series: classification and regression of brain responses using machine learning. Magn Reson Imaging, 26(7), 921-934.

Gauthier, I., Tarr, M. J., Moylan, J., Skudlarski, P., Gore, J. C., \& Anderson, A. W. (2000). The fusiform "face area" is part of a network that processes faces at the individual level. J Cogn Neurosci, 12(3), 495-504.

Goebel, R. (2012). BrainVoyager--past, present, future. Neuroimage, 62(2), 748756.

Grill-Spector, K., Knouf, N., \& Kanwisher, N. (2004). The fusiform face area subserves face perception, not generic within-category identification. Nat Neurosci, 7(5), 555-562. 
Haxby, J. V., Hoffman, E. A., \& Gobbini, M. I. (2002). Human neural systems for face recognition and social communication. Biol Psychiatry, 5l(1), 59-67.

Hoffman, E. A., \& Haxby, J. V. (2000). Distinct representations of eye gaze and identity in the distributed human neural system for face perception. Nat Neurosci, 3(1), 80-84.

Kanwisher, N., McDermott, J., \& Chun, M. M. (1997). The fusiform face area: a module in human extrastriate cortex specialized for face perception. $J$ Neurosci, 17(11), 4302-4311.

Kanwisher, N., \& Yovel, G. (2006). The fusiform face area: a cortical region specialized for the perception of faces. Philos Trans R Soc Lond B Biol Sci, 361(1476), 2109-2128.

Kastner, S., De Weerd, P., Desimone, R., \& Ungerleider, L. G. (1998). Mechanisms of directed attention in the human extrastriate cortex as revealed by functional MRI. Science, 282(5386), 108-111.

Kastner, S., De Weerd, P., Pinsk, M. A., Elizondo, M. I., Desimone, R., \& Ungerleider, L. G. (2001). Modulation of sensory suppression: implications for receptive field sizes in the human visual cortex. $J$ Neurophysiol, 86(3), 1398-1411.

Kastner, S., Pinsk, M. A., De Weerd, P., Desimone, R., \& Ungerleider, L. G. (1999). Increased activity in human visual cortex during directed attention in the absence of visual stimulation. Neuron, 22(4), 751-761.

Kriegeskorte, N., Formisano, E., Sorger, B., \& Goebel, R. (2007). Individual faces elicit distinct response patterns in human anterior temporal cortex. Proc Natl Acad Sci U S A, 104(51), 20600-20605.

Loffler, G., Yourganov, G., Wilkinson, F., \& Wilson, H. R. (2005). fMRI evidence for the neural representation of faces. Nat Neurosci, 8(10), 1386-1390.

Martin, A., \& Chao, L. L. (2001). Semantic memory and the brain: structure and processes. Curr Opin Neurobiol, 11(2), 194-201. 
Mourao-Miranda, J., Bokde, A. L., Born, C., Hampel, H., \& Stetter, M. (2005). Classifying brain states and determining the discriminating activation patterns: Support Vector Machine on functional MRI data. Neuroimage, 28(4), 980-995.

Mur, M., Ruff, D. A., Bodurka, J., Bandettini, P. A., \& Kriegeskorte, N. (2010). Face-identity change activation outside the face system: "release from adaptation" may not always indicate neuronal selectivity. Cereb Cortex, 20(9), 2027-2042.

Nestor, A., Plaut, D. C., \& Behrmann, M. (2011). Unraveling the distributed neural code of facial identity through spatiotemporal pattern analysis. Proc Natl Acad Sci U S A, 108(24), 9998-10003.

Op de Beeck, H. P., Haushofer, J., \& Kanwisher, N. G. (2008). Interpreting fMRI data: maps, modules and dimensions. Nat Rev Neurosci, 9(2), 123-135.

Pinsk, M. A., Arcaro, M., Weiner, K. S., Kalkus, J. F., Inati, S. J., Gross, C. G., et al. (2009). Neural representations of faces and body parts in macaque and human cortex: a comparative FMRI study. J Neurophysiol, 101(5), 2581-2600.

Suckling, J., \& Bullmore, E. (2004). Permutation tests for factorially designed neuroimaging experiments. Hum Brain Mapp, 22(3), 193-205.

van den Hurk, J., Gentile, F., \& Jansma, B. M. (2011). What's behind a face: person context coding in fusiform face area as revealed by multivoxel pattern analysis. Cereb Cortex, 21(12), 2893-2899.

von Kriegstein, K., Kleinschmidt, A., Sterzer, P., \& Giraud, A. L. (2005). Interaction of face and voice areas during speaker recognition. $J$ Cogn Neurosci, 17(3), 367-376.

Weiner, K. S., \& Grill-Spector, K. (2010). Sparsely-distributed organization of face and limb activations in human ventral temporal cortex. Neuroimage, 52(4), 1559-1573.

Weiner, K. S., \& Grill-Spector, K. (2011). Neural representations of faces and limbs neighbor in human high-level visual cortex: evidence for a new organization principle. Psychol Res. 
Weiner, K. S., \& Grill-Spector, K. (2012). The improbable simplicity of the fusiform face area. Trends Cogn Sci, 16(5), 251-254.

Zimmermann, J., Goebel, R., De Martino, F., van de Moortele, P. F., Feinberg, D., Adriany, G., et al. (2011). Mapping the organization of axis of motion selective features in human area MT using high-field fMRI. PLoS One, 6(12), e28 



\section{Chapter 5}

\section{Structural Pathway Connects Anterior Inferior Temporal Cortex With Precuneus and Hippocampus: a DTI Tractography Study ${ }^{4}$}

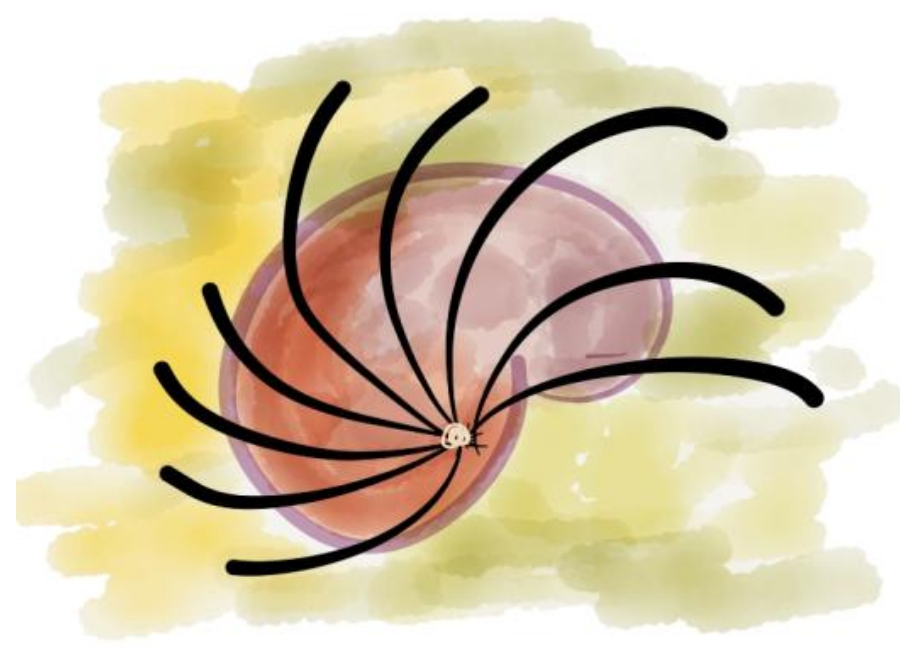

${ }^{4}$ Van den Hurk, J., Bastiani M., \& Jansma, B. M. Structural Pathway Connects Anterior Inferior Temporal Cortex With Precuneus and Hippocampus: a DTI Tractography Study. In preparation. 


\section{Abstract}

Although the involvement of several brain regions in the processing of faces and face identity has been established, the underlying structural connections that facilitate the face recognition network are not very well understood. Recent advances in cognitive neuroscience have led to a new perspective on FFA, both anatomically and functionally (see Chapter 4). FFA can be segregated in a mid fusiform and posterior fusiform face cluster, with both clusters likely serving a different role in the processing of face identity. In addition, it has been shown that the precuneus may play a mediating role during the processing of semantic aspects of identity, in an interplay with fusiform face regions (see Chapter 3). These new findings increase the need for an investigation of the underlying white matter bundles that connect these regions. In the current experiment, we localized face sensitive regions on fusiform cortex using fMRI. In addition, we used diffusion tensor imaging (DTI) and deterministic fiber tractography to map the tracts that connect the fusiform face regions, the anterior inferior temporal lobe (aIT) and the precuneus. Our results show that both mid and posterior fusiform face clusters are connected to aIT, but not to precuneus. From aIT, it seems that two fiber tracts connect with the precuneus: a lateral tract and a medial tract that runs via the hippocampus. These results shed a new light on the potential network that serves the association between faces and related semantic identity information. 


\subsection{Introduction}

The recognition and identification of faces involves a diversity of brain regions (Gobbini \& Haxby, 2007; Haxby, Hoffman, \& Gobbini, 2000, 2002). Among these regions, two are believed to be involved in the visual processing of face features: the Occipital Face Area (OFA; Gauthier, et al., 2000) and the Fusiform Face Area (FFA; Kanwisher, McDermott, \& Chun, 1997; Kanwisher \& Yovel, 2006; Sergent, Ohta, \& MacDonald, 1992). More advanced processing is believed to take place in more distant temporal regions, such as the processing of invariant facial features in the superior temporal sulcus (STS; Haxby, et al., 2000; Hoffman \& Haxby, 2000) and retrieval of (visual) identity information from anterior inferior temporal cortex (aIT; Kriegeskorte, Formisano, Sorger, \& Goebel, 2007).

Notably, white matter connections that underlie this face processing network have received little attention in research. To some extent this is due to methodological limitations: susceptibility artifacts in diffusion imaging often lead to distortions in ventral temporal cortex (Bellgowan, Bandettini, van Gelderen, Martin, \& Bodurka, 2006; Merhof, Soza, Stadlbauer, Greiner, \& Nimsky, 2007) and conclusions based on primate data are not easily transferrable to humans (Gschwind, Pourtois, Schwartz, Van De Ville, \& Vuilleumier, 2012; Tsao, Moeller, \& Freiwald, 2008). In despite of these limitations, a recent diffusion tensor imaging study by Gschwind and colleagues (2012) targeted the structural connectivity between three regions in the face processing network: FFA, OFA and STS. They showed that the interconnections between regions in this 'core system' are not as straightforward and direct as one would expect based on theoretical models of face perception (Haxby, et al., 2000). While providing important new insights in the structural architecture of this core system, this study did not focus on regions that take part in the 'extended system' of face processing (Gobbini \& Haxby, 2007; Haxby, et al., 2000, 2002), including anterior inferior temporal cortex, limbic system and frontal regions. This extended system is involved in more complex aspects of face recognition, such as identity processing.

Two recent insights in the field have raised the need for an investigation of the structural connections that underlie face identification. First, a recent study by Van den Hurk and colleagues (submitted; see Chapter 3) showed that the precuneus, a region located on the medial wall of the parietal lobe, engages in a functional interplay with FFA during the processing of semantic information that is learned in relation to a face. It is still unknown whether a direct connection 
between precuneus and FFA exists, or whether the observed interaction is mediated by other regions. Second, recently the traditional anatomical boundaries of FFA have been redefined into a mid fusiform (mFus) and a posterior fusiform (pFus) face region (Pinsk, et al., 2009; Weiner \& GrillSpector, 2010, 2011, 2012). Next to this anatomical redefinition, it has been demonstrated that mFus and pFus have different contributions to the processing of visual and semantic identity information (van den Hurk, et al., submitted; see Chapter 4). This segregation of FFA raises the question whether these clusters have different structural connections with other regions in the face processing network. In turn, the investigation of the connectivity between the mid- and posterior fusiform clusters with aIT would give more insight in how these regions interact in the context of face identification. In addition, the potential connectivity between the fusiform face regions and the precuneus, possibly with aIT serving as intermediating semantic hub (Patterson, Nestor, \& Rogers, 2007) would information how we associate faces with underlying semantic identity and how that involves fusiform cortex (van den Hurk, et al., submitted; see Chapter $3)$.

In the current study, we intent to delineate the structural network that underlies face identity processing. Based on what has been established about the role of anterior inferotemporal cortex (aIT) in retrieval of semantic knowledge and face identification (Brambati, Benoit, Monetta, Belleville, \& Joubert, 2010; Evans, Heggs, Antoun, \& Hodges, 1995; Gainotti, Barbier, \& Marra, 2003; Grabowski, et al., 2001; Patterson, et al., 2007; Tranel, 2006) and the involvement of the precuneus in processing face identity (Fox, Moon, Iaria, \& Barton, 2009; Gobbini \& Haxby, 2006, 2007; Gobbini, Leibenluft, Santiago, \& Haxby, 2004; Kosaka, et al., 2003) we hypothesize that these regions may be part of a cortical network together with face sensitive regions. Moreover, previous studies have shown that both aIT and precuneus interact with fusiform face regions during retrieval or association of person specific information (Kriegeskorte, et al., 2007; van den Hurk, et al., submitted; van den Hurk, Gentile, \& Jansma, 2011). In the current study, we first localize the fusiform face clusters by using an independent functional localizer run. Then, on an individual subject's basis, we anatomically segment the precuneus and aIT bilaterally. Finally, we employ ROI-based deterministic fiber tracking to investigate the possible white matter connections between the fusiform face regions, the precuneus and aIT. The underlying rationale is that if we find such connections, they would be additional support for the idea of functional interaction between anatomically connected areas of interest. 


\subsection{Materials and methods}

\subsubsection{Participants}

Ten (mean age $=24,6$ females) healthy Dutch speaking participants gave their written informed consent and participated in the study. One of the participants was excluded from the analysis due to technical issues during acquisition of the diffusion MRI data. None of the participants had a history of neurological abnormalities. Approval for the study was granted by the Ethical Committee of the Faculty of Psychology and Neuroscience at the University of Maastricht.

\subsubsection{Procedure}

The participants were placed comfortably in the scanner and their head was fixed with soft foam pads. Participants saw the stimuli projected on a screen through a mirror mounted on the head coil. Stimulus presentation was synchronized with MR data acquisition by triggering the stimulus program (Presentation Software, Neurobehavioral Systems Inc, Albany, CA) with the first MR pulse.

For the functional localizer, we used grey scale images of faces, scrambled faces and houses. Participants were asked to pay close attention to the stimuli. Stimuli were presented in blocks of 20 images with a stimulus duration of $1500 \mathrm{~ms}$ and a $300 \mathrm{~ms}$ inter stimulus interval. Each condition was presented 5 times.

\subsubsection{Data acquisition}

All measurements were performed at a 3-T Siemens Magnetom Allegra head scanner (Siemens Medical System, Erlangen, Germany) using a standard head coil. For localization of face sensitive regions, twenty-five T2*-weighted echoplanar imaging $(\mathrm{EPI})$ slices (voxel size $=2 \times 2 \times 2 \mathrm{~mm}$, inter slice gap $=0 \mathrm{~mm}$ ) covering the temporal lobe were recorded (repetition time $[\mathrm{TR}]=1800 \mathrm{~ms}$, matrix size $=128 \times 128,[\mathrm{TE}]=30 \mathrm{~ms})$.

For structural connectivity analysis, sixty-five diffusion-weighted imaging slices (voxel size $=2,2 \times 2,2 \times 2,2 \mathrm{~mm}$, inter slice gap $=0 \mathrm{~mm}$ ) orthogonal to the scanner axis covering the entire cortical volume were obtained $([\mathrm{TR}]=8100 \mathrm{~ms},[\mathrm{TE}]=91 \mathrm{~ms})$. In total, 41 diffusion-encoding gradient directions with a b-value of $1000 \mathrm{~s} / \mathrm{mm}^{2}$ were used. After each block of 11 images a b0-image (without diffusion-weighting) was obtained. 
In addition to the functional and diffusion images, a high resolution $(1 \times 1 \times 1$ $\mathrm{mm}$ ) T1-weighted anatomical scan was obtained using an ADNI sequence ([TR] $=2250 \mathrm{~ms} ;[\mathrm{TE}]=2.6 \mathrm{~ms}$; flip angle $=9^{\circ}$ ).

\subsubsection{Functional data analysis and ROI definition}

Prior to analysis of the functional data, images were preprocessed using BrainVoyager QX 2.2 (Brain Innovation, Maastricht, The Netherlands). The first 2 volumes were excluded from analysis due to possible T1-saturation. The remaining volumes were corrected for slice scan-time inaccuracies using cubic spline interpolation, and for 3-dimensional head motion using trilinear and sinc interpolation. Finally, linear trends and low frequency temporal drifts were removed from the data using a 6 cycles per run high-pass filter.

In order to localize face selective clusters in ventral temporal cortex, a general linear model (GLM) analysis was conducted on the time series of the functional data (hemodynamic response in BOLD signal change). For each participant, a design matrix was created using a predictor of the time series related to each of the three conditions (faces, houses, scrambled faces). To account for the hemodynamic response delay, the predicted time courses were convolved with a canonical (double gamma) hemodynamic response function (HRF). Subsequently, a conjunction analysis between [faces $>$ houses] and [faces $>$ scrambled faces] was considered (False Discovery Rate (FDR) corrected at $q<$ 0.05). In some participants, not all 4 target clusters appeared at this threshold. In this case, the threshold was lowered until all regions were visible, provided that the condition $[p<0.05$, uncorrected for multiple comparisons] remained satisfied.

To investigate the potential connections between fusiform face regions, aIT and precuneus, individual regions-of-interest (ROIs) were defined in each subject's native brain space. For each subject, the location of the fusiform face regions was based on the results of the localizer. Both the precuneus and aIT were defined individually based on their T1 weighted volumes (see figure 5.1). 


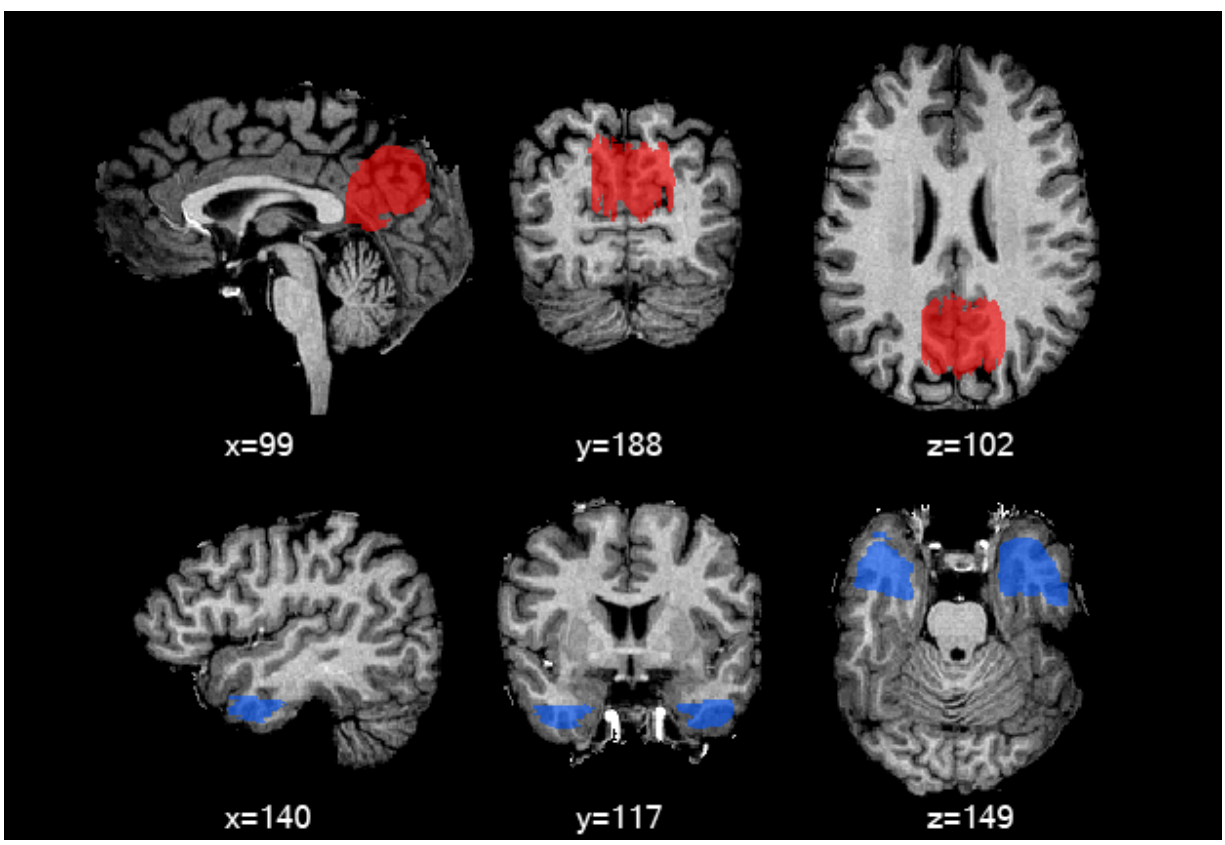

Figure 5.1. Anatomically defined regions-of-interest. For each subject individually, anterior inferior temporal cortex and precuneus were manually segmented (slices presented in native subject coordinates).

\subsubsection{Diffusion-weighted data analysis and ROI-based fiber tracking}

The acquired diffusion images were first co-registered with the high-resolution anatomical data and transformed to the subject's native 3D-space. This step involves resampling of the data to $2 \times 2 \times 2 \mathrm{~mm}$ voxels by using sinc interpolation. Then, the diffusion tensors were estimated at each voxel, based on the raw diffusion measurements and the corresponding direction information. To exclude redundant voxels outside the brain from tensor estimation, a whole brain mask was created to mask the irrelevant voxels out.

To investigate the structural connections between the regions of interest, we employed a ROI-based deterministic fiber tracking algorithm as implemented in BrainVoyager QX 2.4. Fiber tracking is performed in 3D space by using a selective eigenvector interpolation procedure (Kim, et al., 2004). The fibers that connect two ROIs were tracked in two directions: from ROI1 to ROI2 and vice versa. In potential, more fibers are found this way than when using unidirectional tracking. Fibers were tracked in each subject's native brain, as spatial 
transformation of diffusion data is not trivial and can lead to artifacts (Basser \& Jones, 2002). Tracking was performed between all possible ROI-pairs using mFus, pFus, aIT and precuneus, for both hemispheres (seed points per voxel $=3^{3}$ $=27, \mathrm{FA}$ threshold $=0.10$, projection threshold $=0.25$, angle threshold $=50^{\circ}$, step size $=0.5 \mathrm{~mm})$. After tracking, fibers were backprojected to volume (T1) space and transformed to Talairach space for group analysis.

To compare results across subjects, fibers were dilated by $4 \mathrm{~mm}$ after which a 3-dimensional probabilistic map was created in Talairach space. This map reflects the likelihood that fibers connecting two ROIs run through a certain location. This, in turn, gives insight in the existence of a global connectivity network between the ROIs. These resulting maps are thresholded at probability > $30 \%$ and projected on a representative subject's Talairach brain. 


\subsection{Results}

The connectivity pattern between the fusiform face clusters, aIT and precuneus were inspected on consistency across subjects by means of a probabilistic map, thresholded at 30\% (see figure 5.2 and 5.3). Visual inspection of these maps first showed that no direct white matter connections seem to exist between the fusiform face clusters and the precuneus. However, both the mid fusiform and posterior fusiform regions showed a clear connection with aIT through a fiber tract that runs along the inferior part of the temporal lobe, the inferior longitudinal fasciculus (ILF) (see figure 5.4).

From aIT, a prominent white matter bundle connects with the precuneus. Interestingly, near the anterior pole $(y=137)$, the anterior-posterior path divides into two distinct routes: a lateral route and a medial route. The lateral route runs from anterior to posterior in the mid temporal lobe, where it diverts into the arcuate fasciculus (Gschwind, et al., 2012). At the junction of the parietal and temporal lobes, near the supramarginal gyrus, a bundle splits from the main tract to connect with the precuneus. Moreover, this lateral route appears to cross the ILF path connecting fusiform regions and aIT. The second white matter tract that originates from aIT, the medial route runs directly through medial temporal cortex (possibly hippocampus (Bronen \& Cheung, 1991), see figure 5.3 and figure 5.5), a brain region with an important role in memory formation and recollection (Daselaar, Fleck, \& Cabeza, 2006; Ranganath, 2010; Ranganath, Heller, Cohen, Brozinsky, \& Rissman, 2005). From there, the bundle continues its way via the retrosplenial cortex into the precuneus. See figure 5.2 and 5.3 for an overview. 


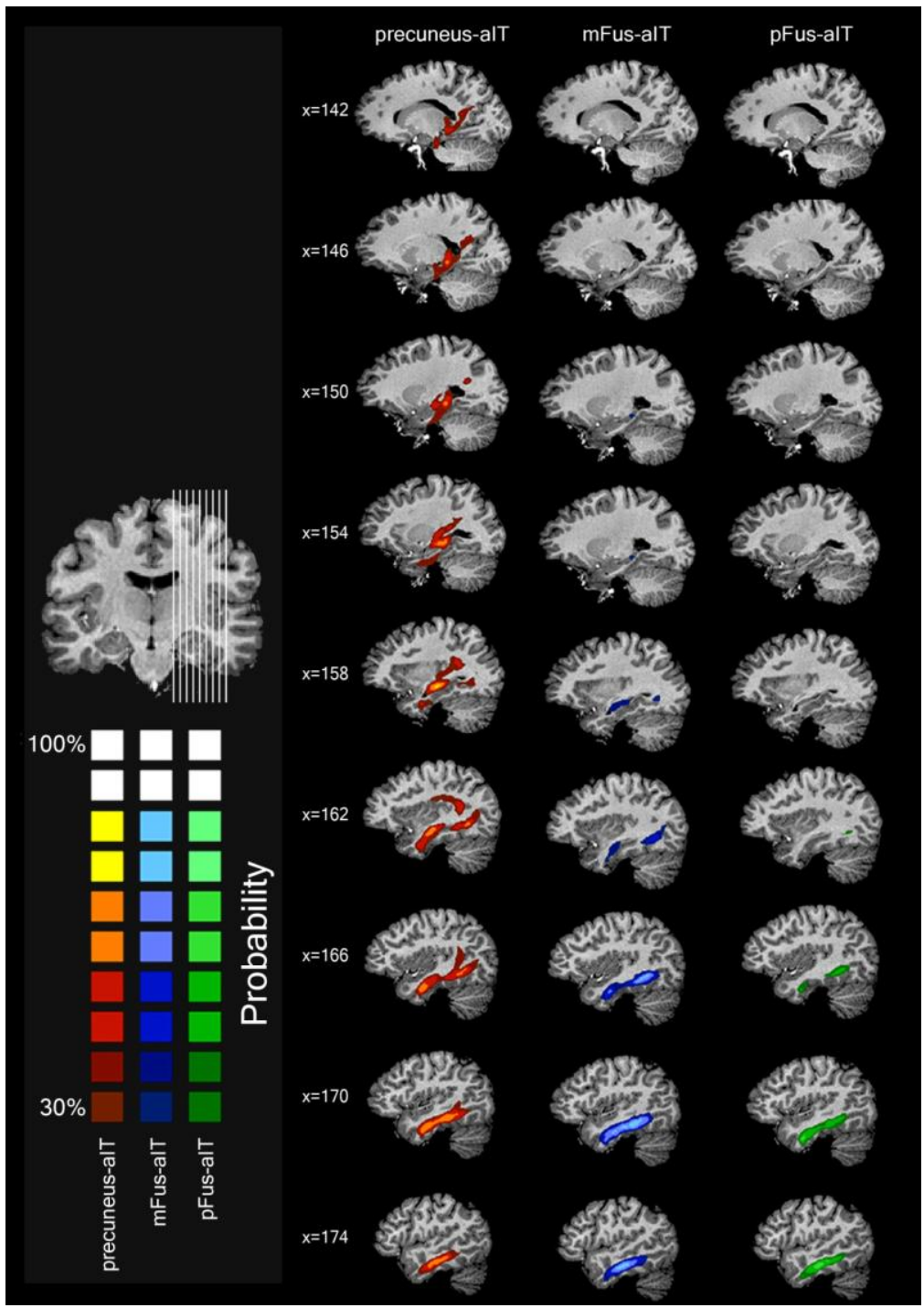

Figure 5.2. Bidirectional ROI connectivity. The white matter projections between precuneus and aIT (left column), mid fusiform face region and aIT (middle column) and posterior fusiform face region and aIT (right column) superimposed on a representative brain in Talairach space. The three tracked pathways are presented individually on 9 sagittal slices, arranged from medial (top) to lateral (bottom). Probability is color coded in accordance with the legend on the bottom left of the figure, with separate colors for the precuneus - aIT (red-yellow), mFus - aIT (blue-white) and pFus - aIT (green-white) connections. Note that only the left hemisphere is shown, but that the projected connections are mirrored in the contralateral hemisphere. Images are in radiological convention, i.e. left is right. 


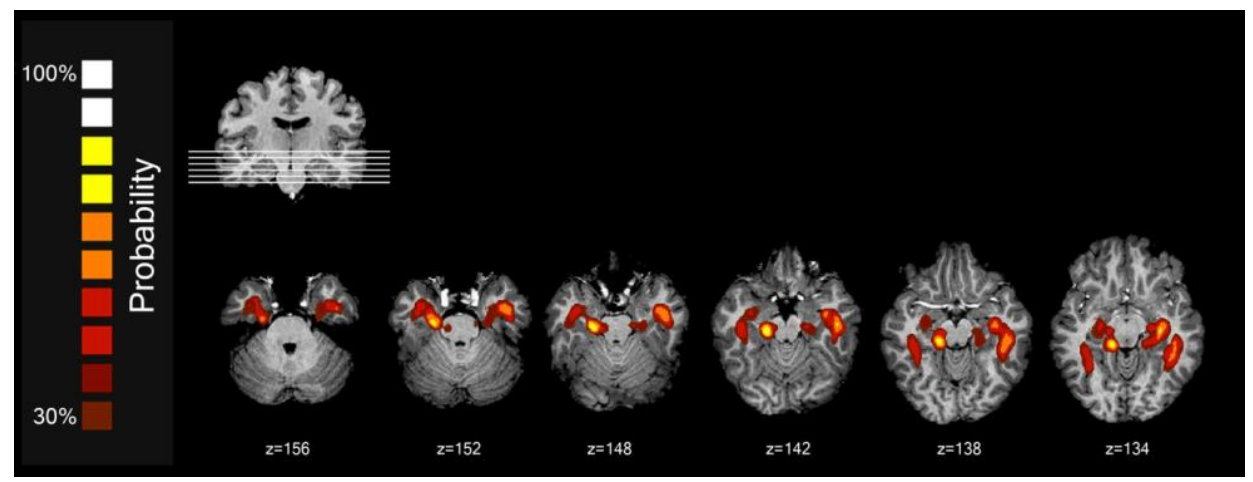

Figure 5.3. Structural connectivity between aIT and precuneus. The probabilistic map is projected on 6 transverse slices. As can be seen, the fiber tracts originating in aIT split into two distinct bundles: a medial and a lateral path. The medial path later extends into hippocampal structures, the lateral path follows the temporal lobe (ILF) in posterior direction, after which it diverts into the arcuate fasciculus. Images are in radiological convention, i.e. left is right.

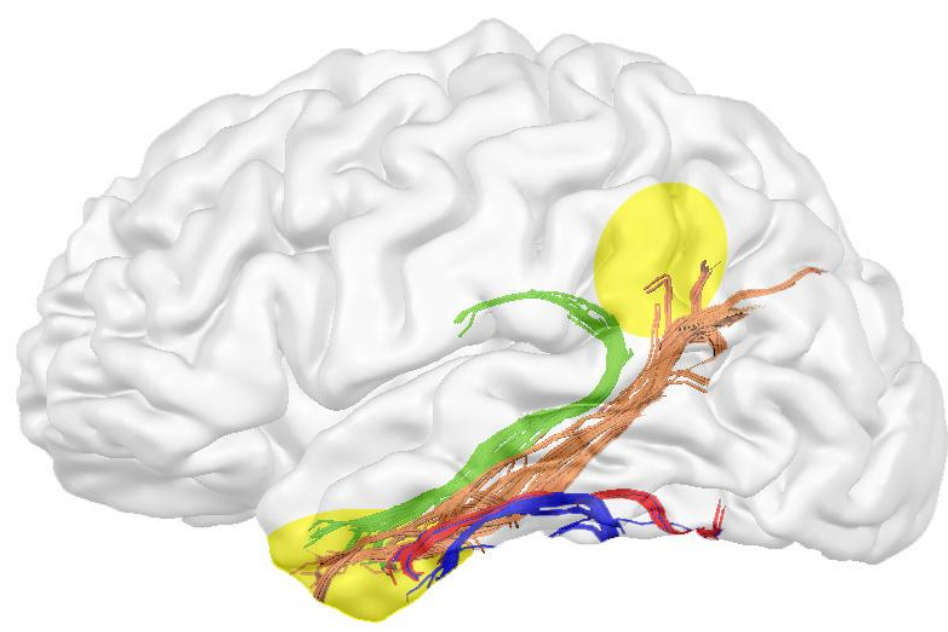

Figure 5.4. Left hemispheric connectivity reconstruction between aIT and precuneus (marked in yellow) in one representative subject. As can be seen, $m F u s$ (blue fibers) and pFus (red fibers) have unique connections with aIT. From aIT, the deterministic tracking algorithm identified two distinct routes that connect with the precuneus. The lateral route (orange) runs via the inferior longitudinal fasciculus, the medial route (green) diverts via medial temporal structures into the arcuate fasciculus. Note that the precuneus is located on the medial side of the brain, not visible from the lateral perspective presented here. See also figure 5.5. 


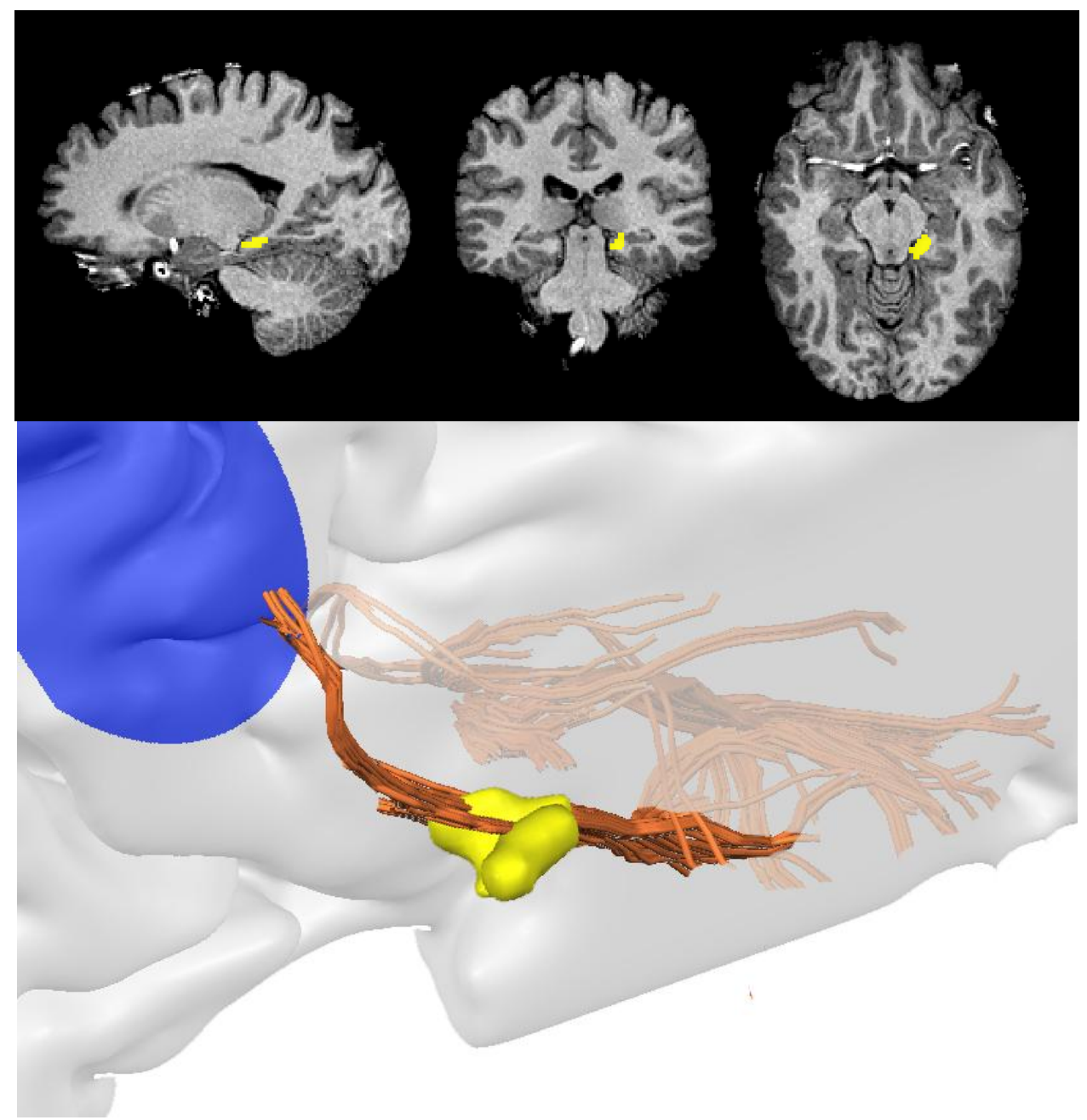

Figure 5.5. Left hemispheric connectivity reconstruction of medial route in one representative subject. Top panel: to demonstrate that the medial route runs via the hippocampus, this region is anatomically defined in the anatomical volume (top figure, yellow voxels) of the subject (Bronen \& Cheung, 1991). Images are in radiological convention, i.e. left is right. Bottom panel: in 3D space, the orientation of the medial pathway is displayed. As can be seen, the fibers that connect the precuneus (blue oval) with aIT (not displayed here) via the medial route directly cross the cluster marked as belonging to the hippocampus (yellow cluster). 


\subsection{Discussion}

In the current study, we investigated the structural network that underlies face identity processing by combining functional MRI and DTI. We localized face sensitive regions on the fusiform gyrus for each participant individually. In addition, we segmented the precuneus and anterior inferior temporal cortex (aIT) for each participant. Then, using a deterministic fiber tracking algorithm, we mapped the white matter bundles that connect these regions. We explicitly targeted aIT, since its role in processing semantic and identity information has repeatedly been demonstrated by various studies (Brambati, et al., 2010; Evans, et al., 1995; Gainotti, et al., 2003; Grabowski, et al., 2001; Patterson, et al., 2007; Tranel, 2006). In addition, we focused on the precuneus because of its established sensitivity to face identity information (Fox, et al., 2009; Gobbini \& Haxby, 2006, 2007; Gobbini, et al., 2004; Kosaka, et al., 2003). Both regions have been shown to engage in an interaction with fusiform face regions during tasks that involve active processing of person specific information (Kriegeskorte, et al., 2007; van den Hurk, et al., submitted; van den Hurk, et al., 2011).

Our results show a clear connection between both mid- and posterior fusiform regions and aIT. However, we could not find a direct pathway between these fusiform regions and the precuneus. Therefore, it is likely that a potential exchange of information between the fusiform regions and the precuneus has to be mediated via a third region. In line with this assumption, there appears to be a dual connection between aIT and the precuneus. This connection consists of a lateral route that runs via the mid temporal lobe into the arcuate fasciculus (Gschwind, et al., 2012). The second tract that originates in aIT, the medial route, diverts into the medial temporal lobe (possibly hippocampus) and continues via the retrosplenial cortex into the precuneus.

Based on these findings we propose a working model for functional interaction between regions relevant to associate faces with biographical background information (see figure 5.4). This model holds especially for the cognitive process of information associations (task in experiments of preceding chapters). In addition, this model is based on plausibility in linking observed anatomical connections to observed predictive coding pattern in certain regions of interest. It therefore should be seen as a working hypothesis for future effective connectivity measures (Friston, Harrison, \& Penny, 2003; Roebroeck, Formisano, \& Goebel, 2011). 


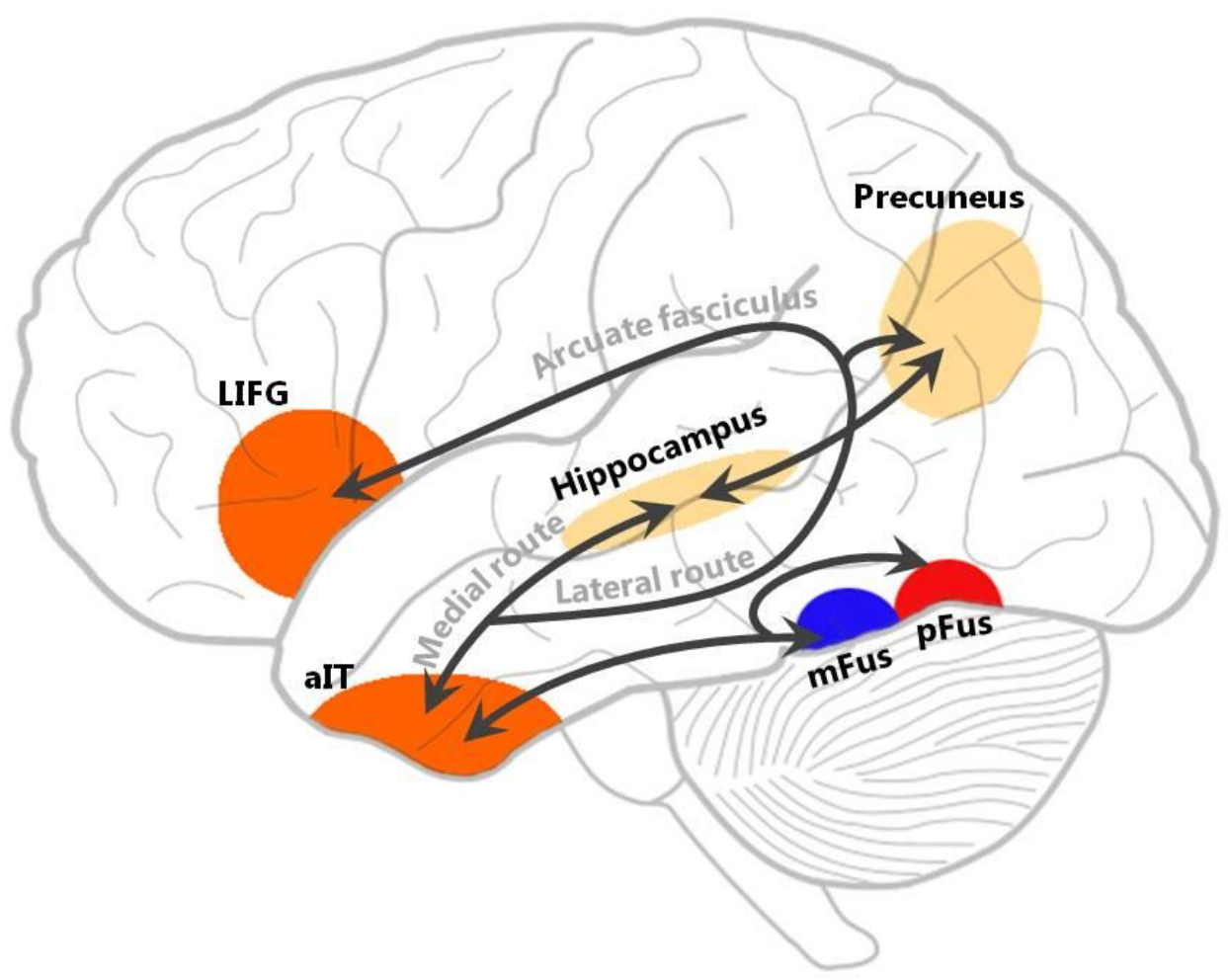

Figure 5.4. Overview of structural connectivity between regions involved in facebiographic knowledge association network. The model as proposed above describes the empirically demonstrated connections between mid- and posterior fusiform face regions ( $m F u s$ and pFus respectively), anterior inferior temporal cortex (aIT), hippocampus, precuneus and (left) inferior frontal gyrus (LIFG). Both $m F$ us and pFus are directly connected to aIT but no direct connection seems to exist with precuneus. Between aIT and precuneus, however, two pathways exist. A medial path runs via medial temporal structures (hippocampus) directly towards the precuneus. A lateral pathway runs into the arcuate fasciculus, which descends into frontal regions. See text in section 5.4 for details.

The lateral route from aIT to precuneus runs partially via the arcuate fasciculus. This dense fiber tract connects temporal structures with prefrontal cortex (Catani, Jones, \& ffytche, 2005; Rilling et al., 2008) which, among other executive functions, is involved in working memory processes (Martin \& Chao, 2001). With regard to the lateral route, it seems plausible that via the arcuate fasciculus the fusiform-aIT network is connected to frontal lobe regions related to visual and verbal working memory. This, in turn, is in accordance with the 
findings described in Chapter 3: the left inferior frontal gyrus (LIFG) is identified by the searchlight as one of the clusters that differentiates between a person and a category semantic context. (see Chapter 3).

The connection between precuneus and hippocampus has been demonstrated in previous work (Blatt, Pandya, \& Rosene, 2003; Margulies, et al., 2009; Suzuki \& Amaral, 1994; Vogt, Vogt, \& Laureys, 2006), and the extension of this fiber bundle towards aIT provides an interesting perspective on the functional role that these structures fulfill within a network: together with the prominent connections between fusiform regions and aIT, the structural architecture as demonstrated in this study is fully in line with the notion that aIT functions as a semantic 'hub' that assembles (semantic) information for various processes (Patterson, et al., 2007). In this view, the precuneus plays a role in the association of face and identity information, as has been demonstrated before (Kottlow, Jann, Dierks, \& Koenig, 2012; von Kriegstein, Kleinschmidt, Sterzer, $\&$ Giraud, 2005). Via an interaction with memory structures as the hippocampus, the precuneus is expected to retrieve (semantic) information related to identity. This information is then likely assembled by aIT and fed to the fusiform face regions. Such an interplay would explain why we observed predictive coding for semantics in FFA (van den Hurk, et al., 2011; van den Hurk, et al., submitted; see Chapter 3; von Kriegstein, et al., 2005). The finding that the neural tuning of the mid fusiform and posterior fusiform face regions is different in the context of identity processing (van den Hurk, et al., submitted; see Chapter 4), may be related to the type of anatomical connectivity as well. More specifically, we hypothesize that feedback information originating from aIT is not sent to both mFus and pFus. Rather, regarding the architecture of the ventral visual stream (Lamme, Super, \& Spekreijse, 1998), one possibility could be that pFus receives the bottom-up visual information about the face and feeds it through the aforementioned network. This network, with a core based on precuneus hippocampal interactions, retrieves associated identity information which is subsequently fed back to the mFus cluster. In contrast, when a semantic description about a known identity is provided, the same network is automatically activated, this time to retrieve the visual information of the corresponding face. This activated face representation is fed via the established aIT - fusiform cortex connection to pFus. This hypothetical scenario is in line with verbal accounts of subjects engaged in a face-semantics association task, where they report to experience an automatic memorization of a face/conceptual identity when being presented with a written description about a face or an image of a face, respectively (van den Hurk, et al., submitted; see Chapter 4). It is also in accordance with studies that show that the fusiform face region (still referred 
to as FFA) is sensitive to the identity of a person (Andrews \& Ewbank, 2004; Barton, Press, Keenan, \& O'Connor, 2002; Gauthier, et al., 2000; Grill-Spector, Knouf, \& Kanwisher, 2004; Haxby, et al., 2002; Hoffman \& Haxby, 2000; Loffler, Yourganov, Wilkinson, \& Wilson, 2005).

It should be noted that in the current experiment we used a voxel size of $\pm 2 \times 2 \times 2 \mathrm{~mm}$, which samples the diffusion across thousands of fibers. Hence, this measure represents a noisy average of the sampled voxel volume. One therefore has to interpret the results with some caution. First, as with other imaging methods, the inability to identify a structural pathway between two regions is by itself not compelling evidence that such a connection does not exist in the brain. Still, the leading notion is that this lack of evidence reflects meaningful anatomical information (Johansen-Berg \& Rushworth, 2009). Second, it should be noted that observing white matter connections only indirectly suggests neural communication between these regions of interest (based on plausibility). Such an assumption has to be tested directly via effective connectivity studies in the future, or by using high temporal resolution techniques sensitive to the time course of information processing (such as EEG, ERP, or an interference method like TMS). 


\subsection{Conclusion}

Recent studies demonstrated that face sensitive regions on ventral temporal cortex are involved in the processing of non-visual identity information. The intention of this study was to examine the structural connections between the fusiform face clusters and two regions that have received little attention in structural connectivity studies in this domain: the anterior temporal pole (aIT) and the precuneus. By using diffusion tensor imaging (DTI), we were able to show that both the mid-fusiform and the posterior fusiform face clusters are structurally connected to aIT. In addition, we delineated a seeming dual pathway between aIT and the precuneus. One pathway runs via a medial route through the hippocampus, a brain region with important memory related functionality. In addition, we observe a lateral route that connects with the arcuate fasciculus, runs into precuneus and extends into the frontal lobe with related memory functions. These findings shed an important light on the white matter architecture that facilitates the elegant interplay between brain regions involved in person identification. Overall this connectivity network suggests a relevant role for aIT as central semantic hub and it suggests that the semantic knowledge representation observed within fusiform face clusters may well be based on a top-down information flow from aIT to these regions. 


\subsection{Acknowledgements}

The authors would like to thank Inge Timmers, Francesco Gentile and Joao Correia for helpful suggestions and comments. 


\subsection{References}

Andrews, T. J., \& Ewbank, M. P. (2004). Distinct representations for facial identity and changeable aspects of faces in the human temporal lobe. Neuroimage, 23(3), 905-913.

Barton, J. J., Press, D. Z., Keenan, J. P., \& O'Connor, M. (2002). Lesions of the fusiform face area impair perception of facial configuration in prosopagnosia. Neurology, 58(1), 71-78.

Basser, P. J., \& Jones, D. K. (2002). Diffusion-tensor MRI: theory, experimental design and data analysis - a technical review. NMR Biomed, 15(7-8), 456-467.

Bellgowan, P. S., Bandettini, P. A., van Gelderen, P., Martin, A., \& Bodurka, J. (2006). Improved BOLD detection in the medial temporal region using parallel imaging and voxel volume reduction. Neuroimage, 29(4), 12441251.

Blatt, G. J., Pandya, D. N., \& Rosene, D. L. (2003). Parcellation of cortical afferents to three distinct sectors in the parahippocampal gyrus of the rhesus monkey: an anatomical and neurophysiological study. J Comp Neurol, 466(2), 161-179.

Brambati, S. M., Benoit, S., Monetta, L., Belleville, S., \& Joubert, S. (2010). The role of the left anterior temporal lobe in the semantic processing of famous faces. Neuroimage, 53(2), 674-681.

Bronen, R. A., \& Cheung, G. (1991). MRI of the normal hippocampus. Magn Reson Imaging, 9(4), 497-500.

Catani, M., Jones, D. K., \& ffytche, D. H. (2005). Perisylvian language networks of the human brain. Ann Neurol, 57(1), 8-16.

Daselaar, S. M., Fleck, M. S., \& Cabeza, R. (2006). Triple dissociation in the medial temporal lobes: recollection, familiarity, and novelty. $J$ Neurophysiol, 96(4), 1902-1911.

Evans, J. J., Heggs, A. J., Antoun, N., \& Hodges, J. R. (1995). Progressive prosopagnosia associated with selective right temporal lobe atrophy. A new syndrome? Brain, 118 ( Pt 1), 1-13. 
Fox, C. J., Moon, S. Y., Iaria, G., \& Barton, J. J. (2009). The correlates of subjective perception of identity and expression in the face network: an fMRI adaptation study. Neuroimage, 44(2), 569-580.

Friston, K. J., Harrison, L., \& Penny, W. (2003). Dynamic causal modelling. Neuroimage, 19(4), 1273-1302.

Gainotti, G., Barbier, A., \& Marra, C. (2003). Slowly progressive defect in recognition of familiar people in a patient with right anterior temporal atrophy. Brain, 126(Pt 4), 792-803.

Gauthier, I., Tarr, M. J., Moylan, J., Skudlarski, P., Gore, J. C., \& Anderson, A. W. (2000). The fusiform "face area" is part of a network that processes faces at the individual level. J Cogn Neurosci, 12(3), 495-504.

Gobbini, M. I., \& Haxby, J. V. (2006). Neural response to the visual familiarity of faces. Brain Res Bull, 71(1-3), 76-82.

Gobbini, M. I., \& Haxby, J. V. (2007). Neural systems for recognition of familiar faces. Neuropsychologia, 45(1), 32-41.

Gobbini, M. I., Leibenluft, E., Santiago, N., \& Haxby, J. V. (2004). Social and emotional attachment in the neural representation of faces. Neuroimage, 22(4), 1628-1635.

Grabowski, T. J., Damasio, H., Tranel, D., Ponto, L. L., Hichwa, R. D., \& Damasio, A. R. (2001). A role for left temporal pole in the retrieval of words for unique entities. Hum Brain Mapp, 13(4), 199-212.

Grill-Spector, K., Knouf, N., \& Kanwisher, N. (2004). The fusiform face area subserves face perception, not generic within-category identification. Nat Neurosci, 7(5), 555-562.

Gschwind, M., Pourtois, G., Schwartz, S., Van De Ville, D., \& Vuilleumier, P. (2012). White-matter connectivity between face-responsive regions in the human brain. Cereb Cortex, 22(7), 1564-1576.

Haxby, J. V., Hoffman, E. A., \& Gobbini, M. I. (2000). The distributed human neural system for face perception. Trends Cogn Sci, 4(6), 223-233. 
Haxby, J. V., Hoffman, E. A., \& Gobbini, M. I. (2002). Human neural systems for face recognition and social communication. Biol Psychiatry, 51(1), 59-67.

Hoffman, E. A., \& Haxby, J. V. (2000). Distinct representations of eye gaze and identity in the distributed human neural system for face perception. Nat Neurosci, 3(1), 80-84.

Johansen-Berg, H., \& Rushworth, M. F. (2009). Using diffusion imaging to study human connectional anatomy. Annu Rev Neurosci, 32, 75-94.

Kanwisher, N., McDermott, J., \& Chun, M. M. (1997). The fusiform face area: a module in human extrastriate cortex specialized for face perception. $J$ Neurosci, 17(11), 4302-4311.

Kanwisher, N., \& Yovel, G. (2006). The fusiform face area: a cortical region specialized for the perception of faces. Philos Trans $R$ Soc Lond B Biol Sci, 361(1476), 2109-2128.

Kim, K. H., Ronen, I., Formisano, E., Goebel, R., Ugurbil, K., \& Kim, D. S. (2004). Robust fiber tracking method by vector selection criterion in diffusion tensor images. Conf Proc IEEE Eng Med Biol Soc, 2, 10801083

Kosaka, H., Omori, M., Iidaka, T., Murata, T., Shimoyama, T., Okada, T., et al. (2003). Neural substrates participating in acquisition of facial familiarity: an fMRI study. Neuroimage, 20(3), 1734-1742.

Kottlow, M., Jann, K., Dierks, T., \& Koenig, T. (2012). Increased phase synchronization during continuous face integration measured simultaneously with EEG and fMRI. Clin Neurophysiol.

Kriegeskorte, N., Formisano, E., Sorger, B., \& Goebel, R. (2007). Individual faces elicit distinct response patterns in human anterior temporal cortex. Proc Natl Acad Sci U S A, 104(51), 20600-20605.

Lamme, V. A., Super, H., \& Spekreijse, H. (1998). Feedforward, horizontal, and feedback processing in the visual cortex. Curr Opin Neurobiol, 8(4), 529-535. 
Loffler, G., Yourganov, G., Wilkinson, F., \& Wilson, H. R. (2005). fMRI evidence for the neural representation of faces. Nat Neurosci, 8(10), 1386-1390.

Margulies, D. S., Vincent, J. L., Kelly, C., Lohmann, G., Uddin, L. Q., Biswal, B. B., et al. (2009). Precuneus shares intrinsic functional architecture in humans and monkeys. Proc Natl Acad Sci US A, 106(47), 2006920074.

Martin, A., \& Chao, L. L. (2001). Semantic memory and the brain: structure and processes. Curr Opin Neurobiol, 11(2), 194-201.

Merhof, D., Soza, G., Stadlbauer, A., Greiner, G., \& Nimsky, C. (2007). Correction of susceptibility artifacts in diffusion tensor data using nonlinear registration. Med Image Anal, 11(6), 588-603.

Patterson, K., Nestor, P. J., \& Rogers, T. T. (2007). Where do you know what you know? The representation of semantic knowledge in the human brain. Nat Rev Neurosci, 8(12), 976-987.

Pinsk, M. A., Arcaro, M., Weiner, K. S., Kalkus, J. F., Inati, S. J., Gross, C. G., et al. (2009). Neural representations of faces and body parts in macaque and human cortex: a comparative FMRI study. J Neurophysiol, 101(5), 2581-2600.

Ranganath, C. (2010). A unified framework for the functional organization of the medial temporal lobes and the phenomenology of episodic memory. Hippocampus, 20(11), 1263-1290.

Ranganath, C., Heller, A., Cohen, M. X., Brozinsky, C. J., \& Rissman, J. (2005). Functional connectivity with the hippocampus during successful memory formation. Hippocampus, 15(8), 997-1005.

Rilling, J. K., Glasser, M. F., Preuss, T. M., Ma, X., Zhao, T., Hu, X., et al. (2008). The evolution of the arcuate fasciculus revealed with comparative DTI. Nat Neurosci, 11(4), 426-428.

Roebroeck, A., Formisano, E., \& Goebel, R. (2011). The identification of interacting networks in the brain using fMRI: Model selection, causality and deconvolution. Neuroimage, 58(2), 296-302. 
Sergent, J., Ohta, S., \& MacDonald, B. (1992). Functional neuroanatomy of face and object processing. A positron emission tomography study. Brain, 115 Pt 1, 15-36.

Suzuki, W. A., \& Amaral, D. G. (1994). Perirhinal and parahippocampal cortices of the macaque monkey: cortical afferents. J Comp Neurol, 350(4), 497533.

Tranel, D. (2006). Impaired naming of unique landmarks is associated with left temporal polar damage. Neuropsychology, 20(1), 1-10.

Tsao, D. Y., Moeller, S., \& Freiwald, W. A. (2008). Comparing face patch systems in macaques and humans. Proc Natl Acad Sci U S A, 105(49), 19514-19519.

van den Hurk, J., Gentile, F., \& Jansma, B. M. (2011). What's behind a face: person context coding in fusiform face area as revealed by multivoxel pattern analysis. Cereb Cortex, 21(12), 2893-2899.

Vogt, B. A., Vogt, L., \& Laureys, S. (2006). Cytology and functionally correlated circuits of human posterior cingulate areas. Neuroimage, 29(2), 452-466.

von Kriegstein, K., Kleinschmidt, A., Sterzer, P., \& Giraud, A. L. (2005). Interaction of face and voice areas during speaker recognition. J Cogn Neurosci, 17(3), 367-376.

Weiner, K. S., \& Grill-Spector, K. (2010). Sparsely-distributed organization of face and limb activations in human ventral temporal cortex. Neuroimage, 52(4), 1559-1573.

Weiner, K. S., \& Grill-Spector, K. (2011). Neural representations of faces and limbs neighbor in human high-level visual cortex: evidence for a new organization principle. Psychol Res.

Weiner, K. S., \& Grill-Spector, K. (2012). The improbable simplicity of the fusiform face area. Trends Cogn Sci, 16(5), 251-254. 



\section{Chapter 6}

General Discussion 
The focus of this thesis was on the potential involvement of the Fusiform Face Area in the processing of semantic identity information, and on probable mechanisms that underlie this processing. In this general discussion, the results and interpretations of the chapters in this work will be reviewed. Also, on a meta level, the consequences as well as alternative interpretations will be discussed.

The first research question that was addressed targeted the possibility that FFA is involved in the processing of semantic identity information, independent of visual face presentation. As many studies in the past years targeted only the visual aspects of identity processing, our findings as presented in Chapter 2 shed a new light on this involvement. The assumed position of FFA in the hierarchical stream of visual object recognition implies that it prefers visual information. As with a many visual processes, complex information may interact with the processing of visual stimuli via top-down connections, but our results show that the patterns of activity in this face selective region can also be modulated by a semantic context in absence of a face. This in turn demonstrates that the functional role of FFA may be more complex than assumed. The strong response to face stimuli that is commonly reported in the literature indeed points towards a sensitivity to faces, but does this automatically imply that the inherent role of neurons within this region is related to mere visual processing? An alternative view is that faces engage FFA because these stimuli ask for identification, more than other categories of visual stimuli. After all, the significance of fast identification of friend and foe is evident from an evolutionary perspective, and whether this identification is based on visual features or not is irrelevant. Visual features are sent to FFA via early visual regions and OFA, such that FFA can initiate the identification process. Its prominent structural connections with anterior inferior temporal cortex, voice regions on the superior temporal gyrus, and emotion networks including the amygdala all suggest a central role in a network for adequate behavioral response initiation, based on important identity information.

In Chapter 2, we focused on the question whether FFA is sensitive to a subtle semantic context manipulation and indeed observed such a sensitivity. In Chapter 3 we extended our view and performed a whole-brain investigation with a similar question: what other brain regions are possibly involved in this semantic processing? After having identified several brain regions, we investigated whether these regions have a functional connection with FFA during this processing. By employing a functional connectivity analysis method, we asked whether some of these regions work together more strongly with FFA during the processing of words presented in a person context than when presented in a categorical context. The only cortical region that met this criterion 
was the precuneus, found on the medial wall of the parietal lobe. Moreover, the strength of functional connectivity between all the clusters that were located, including FFA, was investigated. Interestingly, the only connection that showed a difference in the connectivity strength between the person and category context was the precuneus - FFA link, in favor of the person condition. Not only does this partially resolve the directionality problem that arose in the study described in Chapter 2 (where the significant prediction accuracies did not allow for interpretation towards the person or category condition), it also shows that FFA indeed is recruited during the processing of identity information even without direct visual face information being present. Together with evidence that activity in FFA is directly modulated during the identification of a familiar voice in absence of a face, the results in Chapter 3 illustrate the likelihood that the role of FFA goes beyond mere visual processing.

During the course of this thesis, a series of studies showed that the anatomical definition of FFA can be redefined into two clusters. At a typical voxel size of $\pm 3 \times 3 \times 3 \mathrm{~mm}$ and after a spatial smoothing procedure that is commonly applied when localizing functional brain regions, these two clusters easily blend together into one. In addition, the exact cortical location of FFA shows significant variability across individuals. This has led to a lack of clear anatomical constraints by which FFA can be distinguished from other face sensitive clusters. Recently, the increasing gain in resolution at which activity information can be sampled from the brain has led to the conclusion that FFA consists of (at least) two sub clusters: a mid-fusiform and posterior fusiform face cluster. Chapter 2 and Chapter 3 do not yet take this dissociation into consideration, but in Chapter 4 and 5 the potential difference that these clusters have in functionality and connectivity are investigated.

In Chapter 4 we presented participants with 3 distinct identities while measuring the activity in the mid-fusiform and posterior fusiform face clusters. These identities comprised both a face and a semantic identity description, which were presented separately to the subjects. By using a pattern-recognition algorithm, we were able to decode above chance what face was presented at a given time from activity in the mid-fusiform cluster, but not from the posterior fusiform region. In contrast, the patterns of activity in the posterior cluster reflected information on the semantic identity that the subject was processing when reading words. This functional double dissociation between these clusters and the contribution they have in the processing of identity is of high importance for the understanding of the involvement of ventral temporal cortex in identification processes. However, the interpretation of these results with respect to the functional dedication of the mid- and posterior fusiform regions is not 
trivial. Combining the perspective that the representation of objects and categories increase in complexity from posterior to anterior temporal regions with the task the subjects were performing in the experiment, we argue the following. When a subject sees one of the faces, the corresponding semantic information needs to be retrieved and is associated with support of the midfusiform area to that face at that moment. Vice versa, when the series of words is presented to the subject, visual aspects of the corresponding face is automatically retrieved from memory with support of the posterior fusiform cluster, also with the purpose of associating the relevant face with the corresponding semantic information presented at that moment. This view is in line with accounts of participants who report to experience an automatic retrieval of the face when being presented with the written description of the corresponding person, and an immediate retrieval of semantic information as soon as one of the faces was shown. As described in Chapter 4, we cannot conclusively derive from these results whether the information we decode locally from activity directly corresponds to the stimulus dimension presented to the participants (i.e. faces and semantic descriptions), or whether this reflects (complex) information that is fed back from higher regions. The temporal resolution of fMRI does not allow for making inferences about the chronometry of events and, in despite of the relatively high resolution that we used in this experiment, the resolution of the functional voxels is still too coarse for examination of individual cortical layers and their role in receiving, processing, and sending out information. Future studies may be able to resolve this important issue by combining ultra high field fMRI with pattern recognition techniques to sample information from individual layers in mid-fusiform and posterior fusiform face regions during a task that involves person identification. In despite of these constraints, based on our findings we can conclude that FFA not only requires an anatomical but also a functional redefinition.

As can be seen in Chapter 4, the segregation of FFA into two clusters provided a gain in the accuracy at which information could be decoded from patterns of activity. The accuracy at which we can examine these apparent modules greatly depends on the resolution of the imaging methods that are used. Future developments in neuroimaging techniques may allow for additional divisibility of these fusiform face regions, which in turn can lead to further understanding of their exact functional role.

When interpreting the results from Chapters 2, 3 and 4, the need for an investigation of the structural connections that underlie the integration and processing of faces and related semantic knowledge increases. Therefore, in Chapter 5, we examined the fiber bundles that potentially connect the mid-and 
posterior fusiform face clusters, anterior inferior temporal cortex (aIT), and the precuneus. Our results show a clear connection between both mid- and posterior fusiform regions and aIT. We could not observe possible difference in the structural connectivity between these mid- and posterior fusiform cluster and aIT, eventually related to limits in spatial resolution. It would be interesting to investigate whether the functional dissociation for $\mathrm{mFus}$ and $\mathrm{pFus}$, as observed in Chapter, is reflected in different landing points within aIT. We could not observe a direct pathway between these fusiform regions and the precuneus, which cannot be explained by resolution as we observed tracts well in the participants. Hence, we assume that this lack means that there is no direct functional connections, and the observed functional correlations (Chapter 3) between the fusiform regions and precuneus must be mediated by other areas. Interestingly, there appears to be a dual connection between aIT and the precuneus. This connection consists of a lateral route that runs via the mid temporal lobe, and a medial route that diverts into the hippocampus and continues via the retrosplenial cortex into the precuneus. As discussed in Chapter 5, the fiber bundles that connect aIT with precuneus via the hippocampus provide an interesting perspective on the possible network that underlies the integration of faces and related semantics. Studies that have shown that aIT functions as a semantic 'hub' that assembles relevant semantic information for further processing are in line with the perspective outlined in this thesis. The demonstration that the precuneus and fusiform face clusters engage in a functional interaction during the processing of person-related information is supported by the structural connection between precuneus and the fusiform face regions via aIT. If this is indeed the case, one could speculate about the reason why we did not find aIT in the searchlight analysis in Chapter 3. We expect that a low signal-to-noise ratio (possibly due to susceptibility artifacts) may have caused the searchlight to be 'blind' for possible differences subtle activity differences near aIT.

In summary, the studies reported in this thesis jointly point towards an involvement of the fusiform face regions (previously referred to as FFA) in the processing, association and retrieval of person-related information in absence of direct face information. Both from a functional (Chapter 2 and 4) and a connectional (Chapter 3 and 5) viewpoint, it has been demonstrated that face selective regions in ventral temporal cortex are likely involved in the processing of person identity in a holistic manner. 

The aim of the current dissertation is to broaden our understanding of the functional and structural principles that underlie face and person identity processing. More specifically, its intention is to dissociate between visual and semantic identity representations in this context. In Chapter 2 we asked whether the fusiform face area, or FFA, is sensitive to semantic information about persons in absence of direct face presentation. An $8 * 8$ word matrix was constructed that consisted of 8 categories (professions, capital cities, house types, sports, hobbies, domestic animals, music styles and car brands). From this matrix, a category condition was created by using the items from a single category per block (the columns in the word matrix). Next to this, we created a person condition by using one word from each category (the rows in the word matrix): each resulting sequence of 8 words could be interpreted as a keyword description of an individual profile (i.e. a surgeon living in Amsterdam, playing soccer etc.). By using this approach, we were able to create two distinct semantic contexts while controlling for visual and semantic input of individual items across conditions. Participants were presented with blocks of word from both the category and the person condition while their brain activity was measured using functional MRI. In the persons condition, they were asked to associate the semantic descriptions with a face that was presented some time before the onset of the words. In the category condition, the series of words were to be associated with a picture that corresponded to the category. In addition, we localized FFA in both hemispheres by means of an independent localizer run. During the analysis, we focused on the patterns of activity elicited by the category and person conditions and found them to be significantly different from each other. From this, we concluded that FFA is sensitive to the subtle semantic context manipulation that we used in this experiment. This in turn may suggest that FFA processes semantic information about a person, even when there is no face visible.

Chapter 3 built further on this finding by extending the dataset we used in Chapter 2, and this time we focused on the entire brain. First, by means of a multivariate searchlight technique we searched for local clusters that, like FFA, are sensitive to the semantic person-category manipulation. On a group level, this searchlight resulted in the left inferior frontal gyrus (LIFG), bilateral angular gyri and precuneus. In the second part of this analysis, we asked whether the functional connectivity between these identified clusters and FFA differed for the person- and the category condition. We hypothesized, in light with the findings in Chapter 2, that the functional connectivity would be stronger between FFA 
and (a subset of) the regions identified with the searchlight during the person condition compared to the category condition. Indeed, we found the strength of connectivity to be stronger between FFA and the precuneus during the processing of the person context, but not during the category context. To a lesser extent, this pattern was also apparent between FFA and right angular gyrus. To see whether this effect may have been caused by a general effect induced by the person condition, such as arousal, we also computed the connectivity coefficients between all the identified clusters in a pair-wise fashion. Interestingly, no difference between the person and category conditions was observed for these paired comparisons. We therefore concluded that the functional coupling between FFA and the precuneus is meaningful in the context of person-related semantics. It may very well be that this functional interaction facilitates the association of a face with semantics.

In Chapter 4 we asked whether the information about an identity in FFA (as indicated in Chapter 2) is specific enough to be decoded from patterns of brain activity. More specifically, we attempted to predict which person was presented to the participant at a given time, by merely looking at the patterns of activity in FFA. We thereby employed the notion that FFA can and should be regarded as consisting of (at least) two distinct sub clusters: a mid fusiform (mFus) and a posterior fusiform (pFus) face cluster. We repeatedly presented participants with 3 distinct identities: first a face was presented, followed by a brief semantic description about that identity. We instructed the participants to actively associate the face with the semantics. In addition, we carefully localized the mFus and pFus clusters by means of an independent localizer run. During the analysis, we investigated at which accuracy we could predict the correct face and the semantic description from patterns of activity in mFus and pFus. We demonstrated that we could decode which face was presented from activity in mFus, but not in pFus. Vice versa, we were able to predict above chance level which semantic description was presented from activity in the pFus cluster. Taking into account that the participants were not merely watching the faces and descriptions passively but rather actively associating them with each other, we interpreted these results as follows: during the presentation of the face, the semantic description is automatically retrieved from memory. In contrast, during the presentation of the words, the face is recalled automatically. Therefore, we concluded that the mFus cluster receives semantic information about a face during the association task, whether the pFus cluster processes the visual aspects. We also investigated what gain the separation of the two clusters had on the prediction accuracies. We therefore collapsed the mFus and pFus clusters into traditional FFA, and observed a loss in the accuracy at which we could predict 
the identities. Only the right FFA allowed for decoding the semantic identity, neither left nor right FFA had predictive value for the visual identities. This study was the first to demonstrate a functional segregation within FFA, a functional region long thought to reflect a single processing module.

Chapter 5 intended to identify the structural connections between the brain regions involved in the processing of semantic person identity. The findings described above provide relatively new insights in the role FFA plays in semantic person identity processing: FFA can be segregated in a mid fusiform and posterior fusiform face cluster, with both clusters likely serving a different role in the processing of face identity (Chapter 4). In addition, it has been shown that the precuneus may play a mediating role during the processing of semantic aspects of identity, in an interplay with fusiform face regions (see Chapter 3). These new findings thus increase the need for an investigation of the underlying white matter bundles that connect these regions. Therefore, we localized face sensitive regions on fusiform cortex using fMRI. In addition, we used diffusion tensor imaging (DTI) and a fiber tracking method to map the tracts that connect the fusiform face regions, the precuneus and the anterior inferior temporal lobe (aIT). Our results showed that both mid and posterior fusiform face clusters are connected to aIT, but not to precuneus. From aIT, it seemed that two fiber tracts connect with the precuneus: a lateral tract and a medial tract that runs via the hippocampus. These results shed a new light on the potential network that serves the association between faces and related semantic identity information.

In summary, the studies reported in this thesis jointly point towards an involvement of the fusiform face regions (previously referred to as FFA) in the processing, association and retrieval of person-related information in absence of direct face information. Both from a functional (Chapter 2 and 4) and a connectional (Chapter 3 and 5) viewpoint, it has been demonstrated that face selective regions in ventral temporal cortex are likely involved in the processing of person identity in a holistic manner. 

Deze dissertatie heeft als voornaamste doel om de functionele en structurele processen die ten grondslag liggen aan de verwerking van gezichts- en persoonsidentiteit beter te begrijpen. In het bijzonder wordt hier getracht om de visuele en de semantische aspecten van identiteit gescheiden van elkaar te onderzoeken.

In Hoofdstuk 2 onderzochten we of de 'fusiform face area', afgekort FFA, gevoelig is voor semantische informatie over personen, in de afwezigheid van een gezicht. Hiertoe werd een $8 * 8$ woordmatrix geconstrueerd, bestaande uit 8 categorieën (beroepen, hoofdsteden, huistypes, sporten, hobby's, huisdieren, muziekstijlen en automerken). Deze matrix werd vervolgens gebruikt om een categorieconditie te ontwikkelen: 8 woordreeksen werden gecreëerd door per categorie alle 8 items samen te nemen (de kolommen uit de woordmatrix). Daarnaast werd een persoonsconditie gevormd door 8 woordreeksen te maken, ditmaal door één woord per categorie te gebruiken (de rijen uit de woordmatrix). Elk van deze 8 woordreeksen kon nu geïnterpreteerd worden als een steekwoordelijke beschrijving van een identiteit (bijvoorbeeld: een chirurg die in Amsterdam woont, voetbal speelt, etc.). Met behulp van deze woordmatrix konden we twee afzonderlijke semantische contexten creëren, terwijl we de visuele en de semantische input in beide condities qua woorden gelijk konden houden. Proefpersonen kregen woordreeksen te zien van zowel de categorie- als de persoonscondities, terwijl hun hersenactiviteit werd gemeten met functionele MRI. Hen werd gevraagd om in de persoonsconditie deze semantische beschrijvingen te associëren met een gezicht dat net voor deze woordreeks werd gepresenteerd. In de categorieconditie was het de bedoeling dat de reeks woorden werd geassocieerd met een plaatje dat de betreffende categorie beschreef. Verder werd FFA in beide hersenhelften gelokaliseerd aan de hand van een onafhankelijke run. Tijdens de analyse lag de nadruk op de patronen van activiteit die in FFA werden geïnduceerd door de persoons- en de categorieconditie, en we ontdekten dat deze patronen significant van elkaar verschilden. Hieruit konden we concluderen dat FFA gevoelig is voor de subtiele semantische manipulatie die we hebben gebruikt in dit experiment. Dit kan betekenen dat FFA semantische informatie over personen verwerkt, zelfs als er niet direct een gezicht zichtbaar is.

Hoofdstuk 3 borduurde verder op deze bevindingen door de dataset die we in Hoofdstuk 2 gebruikt hebben uit te breiden, en door dit keer de nadruk te leggen op het hele brein. Eerst hebben we aan de hand van een multivariate 
'zoeklicht'-techniek gezocht naar lokale clusters in het brein die, net als FFA, gevoelig zijn voor de semantische persoon-categorie manipulatie. Op groepsniveau resulteerde deze analyse in de volgende gebieden: linker inferior frontal gyrus, de angular gyri in beide hemisferen en de precuneus. In het tweede deel van deze analyse stelden we de vraag of de functionele connectiviteit tussen deze clusters en FFA verschilde voor de persoons- en voor de categorieconditie. De hypothese was, in overeenstemming met de bevindingen van Hoofdstuk 2, dat de functionele connectiviteit sterker zou zijn tussen FFA en (enkele van) de overige gebieden tijdens de persoonsconditie dan in de categorieconditie. En inderdaad, we ontdekten dat de mate van de connectiviteit tussen de precuneus en FFA hoger was tijdens de persoonsconditie. In mindere mate vonden we ditzelfde patroon terug tussen de angular gyri en FFA. Om te zien of dit effect misschien veroorzaakt zou zijn door een algemeen effect in het brein veroorzaakt door de persoonsconditie, hebben we ook de functionele connectiviteitscoëfficiënten berekend tussen alle mogelijke combinaties van de clusters buiten FFA. Hier werd geen verschil tussen de twee condities ontdekt, wat er op duidt dat de connectiviteit tussen FFA en de precuneus daadwerkelijk te maken heeft met het associëren van gezichten met semantische informatie.

In Hoofdstuk 4 onderzochten we de vraag of de informatie over identiteit in FFA (zoals aangegeven in Hoofdstuk 2) specifiek genoeg is om te kunnen decoderen uit patronen van hersenactiviteit. In andere woorden, we keken of het mogelijk was om te voorspellen welke persoon werd gepresenteerd aan de proefpersoon, door enkel te kijken naar activatiepatronen in FFA. Hierbij gebruikten we de recente ontdekking dat FFA feitelijk bestaat uit (minstens) twee afzonderlijke subclusters: een mid fusiform (mFus) en een posterieure fusiform (pFus) cluster. We lieten proefpersonen herhaaldelijk 3 verschillende identiteiten zien: eerst werd een gezicht gepresenteerd, gevolgd door een korte semantische omschrijving van de bijbehorende identiteit. De proefpersonen waren geïnstrueerd om het gezicht actief te associëren met de semantische informatie. Daarnaast lokaliseerden we nauwkeurig de mFus en pFus clusters aan de hand van een onafhankelijke lokalisatierun. Tijdens de analyse onderzochten we hoe nauwkeurig we konden voorspellen welk gezicht en welke semantische omschrijving er op een gegeven moment werd gepresenteerd, door enkel te kijken naar activatiepatronen in mFus en pFus. Hier lieten we zien dat we, door te kijken naar mFus, konden voorspellen welk gezicht er werd gepresenteerd. Dit bleek echter niet mogelijk door te kijken naar pFus. Vice versa konden we wel voorspellen welke semantische omschrijving werd gepresenteerd door te kijken naar deze pFus cluster, terwijl dit niet mogelijk 
bleek in mFus. Omdat de proefpersonen niet enkel passief naar de gezichten en omschrijvingen keken, maar juist actief het een met het ander aan het associëren waren, hebben we deze bevindingen als volgt geïnterpreteerd: tijdens het zien van het gezicht wordt de semantische informatie automatisch uit het geheugen opgehaald. Op het moment dat het gezicht wordt laten zien, wordt ook automatisch de bijbehorende informatie actief. Daarom concludeerden we dat de mFus-cluster voornamelijk informatie ontvangt over de semantieke aspecten, terwijl de pFus-cluster betrokken is bij de visuele aspecten. Ook werd gekeken of er een toename was in de nauwkeurigheid waarop de identiteitsvoorspellingen konden worden gedaan, nu we keken naar twee afzonderlijke clusters in plaats van naar één FFA. Daarom hebben we de clusters gegroepeerd tot de meer traditionele FFA en gekeken in hoeverre we nu nog identiteitsinformatie konden aflezen uit patronen van activiteit. We ontdekten dat het niet meer mogelijk was om de gezichten te voorspellen, en dat we enkel nog de semantische omschrijvingen konden voorspellen uit de rechter FFA. Deze studie liet dus voor het eerst zien dat er een functionele segregatie is binnen FFA, een gebied waarvan lang werd vermoed dat het slechts één functie had.

Hoofdstuk 5 richtte zich op de structurele verbindingen tussen hersengebieden die betrokken zijn bij het verwerken van semantische persoonsinformatie. De bevindingen die hierboven beschreven zijn geven een relatief nieuwe visie op de rol die FFA speelt in het verwerken van identiteiten: FFA kan worden opgedeeld in twee clusters die elk naar alle waarschijnlijkheid een andere rol spelen (zie Hoofdstuk 4). Daarnaast hebben we laten zien dat de precuneus een rol speelt tijdens het verwerken van semantische aspecten van identiteit, in samenwerking met de fusiforme clusters (zie Hoofdstuk 3). Deze nieuwe ontdekkingen vroegen om een onderzoek naar de anatomische verbindingen tussen de deze gebieden. In dit hoofdstuk hebben we daarom de fusiforme clusters gelokaliseerd met behulp van fMRI. Vervolgens hebben we diffusion tensor imaging (DTI) gebruikt om de verbindingen in kaart te brengen tussen deze fusiform clusters, de precuneus en de anterieure inferotemporaalkwam (aIT). Dit laatste gebied staat bekend als een gebied dat functioneert als een semantische 'hub', en is daarom relevant in deze context. Onze resultaten lieten zien dat zowel mFus als pFus verbonden zijn met aIT, maar niet met de precuneus. Vanuit aIT lopen twee verbindingen naar de precuneus: een laterale verbinding, en een mediale verbinding die via de hippocampus loopt. Deze resultaten laten een nieuwe invalshoek zien in het netwerk dat mogelijk ten grondslag ligt aan de associatie van gezichten met semantische informatie. 
Samenvattend laten de studies in deze dissertatie gezamenlijk zien dat de fusiforme clusters (voorheen FFA genoemd) betrokken zijn bij de verwerking, associatie en het herinneren van persoonsgerelateerde informatie, in de afwezigheid van directe gezichtsinformatie. Zowel vanuit een functioneel (Hoofdstuk 2 en 4) als vanuit een connectionistisch (Hoofdstuk 3 en 5) perspectief is aannemelijk gemaakt dat gebieden die gevoelig zijn voor gezichten in ventro-temporele cortex betrokken zijn bij het verwerken van persoonsidentiteit op een holistische manier. 


\section{Acknowledgements}

Thanks all. I hope I did not forget to mention anybody. 
All right, since you took the effort to turn the previous page, I should spend some words on acknowledgements. I am very grateful to all of you that supported me, helped me, corrected me, cheered me up, and sometimes plainly annoyed me throughout the last four years. Without having all those people standing behind me, this dissertation would have been nothing more than an embarrassing collection of null results and excuses, cheered up with some meaningless color figures.

There would have been no dissertation (and thus no acknowledgement section) at all without someone that risked her career by hiring me. Bernie, I still clearly remember the sunny Friday afternoon in September 2008 when I was on my bike, on my way to a colloquial lecture in the DEB building. I saw you walking in the same direction and, since we were both late, I offered you a ride. On the back of my bike you mentioned the open $\mathrm{PhD}$-position in your group, which I gratefully accepted within a week. Throughout the following four years, you showed to be the best supervisor I could possibly think of. It was an absolute honor to be working with you. You proved to be an expert in many fields and taught me uncountable things about research and, more importantly, about myself. Whenever I ran into your office with that over-excited 'I-have-the-bestidea-ever'-look in my eyes, you were able to bring me back to earth in a few minutes. At the same time, when I lost faith in my data, my findings or my personal skills, you cheered me up and put me back on track with the right words, insights and out-of-the-box ideas. I appreciated your interested in my personal life and the fact that I was always welcome to drop by for a chat, both at your office or at home. I will carry both your endless enthusiasm for science as well as your caring personality with me for the rest of my career. Thanks for everything, Bernie.

My research at this department got seasoned with a twist of Italian enthusiasm. Elia, as my co-promotor, former internship supervisor and head of the department, I learned a lot about research methods, department bureaucracy and what defines good food. Your critical view on my work during progress reports kept me focused on the importance of carefully designed studies and good statistics. After having heard a lot of constructive remarks from your side, I felt encouraged by your finishing '... but it's very interesting'. Your witty sense of humor and your distinguishable laughter down the hallway always cheered me up. And as a roommate of Michelle, I loved hearing your brain storms and discussions with fluctuating sound intensity, because of your automatic 'walking inand out of the office' habit during these ponderings. Thanks a lot! 
In my early days as a $\mathrm{PhD}$ student I would have been lost as a newborn baby in a topless bar, if I wouldn't have had a figurative mentor. Francesco, thanks for being the scientific help desk during those times. I knew I was welcome anytime if I had questions or issues, and you would literally drop your headphones to help me out. In a later stage, your comments and suggestions on my ideas, interpretations and writings improved my work significantly. I recall several times that you cheered me up when things weren't going the way I wanted them to go. Especially when I was working late on stuff, your out-of-tune whistling down the hallway always put a smile on my face. Your way of life, in which you were able to combine your passion for research with your enthusiasm for travel, party and laughter have always been an inspiration for me. Thanks for everything.

It all started in the famous office number 4.777, with my lovely roommates Michelle and Marieke. Decorating our office with Christmas lights and fake plants (leaving them until half July) was standard practice. The same holds for birthday decorations at the ceiling. How many times did colleagues enter our room and ask whose birthday it was? And how many times have you girls warned me that I should clear away those Christmas lights with more care? Dancing on the chairs for music videos, synching our 90's music mix on youtube so we can look at each other at a cool part when listening with head phones, debating about the (non)senses of science, organizing a department weekend... You guys made my office a blast, and it was nothing less than a privilege to share those precious years with you. And Marieke, I miss your chats, giggles and frowns from the very moment you left.

I always brag about how cool all my colleagues are. But a true interbrain phase locking phenomenon occurred with some of these colleagues. Michelle, your cleverness and die hard dedication to science has always made a huge impression on me. In addition, your open and charming personality made it a total pleasure to organize numerous things together. Whether I was complaining about the rain that always started as soon as I left the building, about that tiny mistake that I made in the first step of my analysis two weeks ago, or about random objects left on my desk by a 'random' other colleague: I valued your advices and insights very much. Without any doubt, you definitely improved the quality of my science. Michelle, thanks for your support, your great spirit, and your amazing friendship.

This phase locking principle also applies to Tom, although I dare to say that every now and then our brains switched into perfect anti-phase. It is amazing how accurately we could predict each other's opinion on some issues, just by 
computing [1-(own opinion)]. To me you are the clearest example of a classic scientist. When it comes to science, your enthusiasm, creativity and ideas are plainly impressive. The ease at which you come up with solutions, the speed and quality of your writing and your superior view on how to promote science is something I am truly jealous of. What makes you even more the classic scientist, is your way of clearing your mind. Numerous times have I seen you lying on the ground, balancing on chairs, folded in stools, hitting your head against the window, taking stuff from my desk and move it to yours or leave random objects from the TMS lab on mine. During our joint science trip to Florida I found a great friend with whom I love to have philosophic discussions, a good whisky and play guitar. Tombo, thanks for listening to all my jumbled brain storms, for your constructive comments and your twist of realism that I often needed. Most of all, thanks for your friendship.

As I stated before, my colleagues here at the faculty created an addictive atmosphere that made me come to work with great pleasure every single day. Whether I needed a good laugh or serious advice, I simply knew that I could walk into your offices. I can't thank you enough for that! Martin, your dry, witty humor has proven to be fully compatible with my laughing networks. Thanks for the poker evenings (and hence, your money), lunches, Efteling trips and the awesome day at the Great Wall in China. You are an awesome character (thanks for not making fun of my mispronunciation of that word the last years). Federico, I got to know you already during my Master internship, during which you warned me for the big impact that a $\mathrm{PhD}$ has on your free time. I thought you were exaggerating, but that prediction turned out to be quite accurate in the end. I appreciate your clever mind a lot at work, and I am pretty sure that I can say that I learned the most about MVPA from you (either directly or indirectly). Outside work, I enjoyed your sense of humor, your awesome cooking and your man-hugs to make Tom uncomfortable. Your hospitality will be never forgotten (can I stay at your place as soon as I live somewhere else?). Giancarlo, what would I have done without your methodological insights? Even my data complained about wanting to be normal, and your soothing words on non-parametric tests and permutations made it feel better again. You are one of the kindest persons I have ever met, and it was my total pleasure to have worked and laughed with you. Kamil, you proved to me that being an amazing scientist with high impact can be combined with being a jolly bloke with a great taste for wine, a passion for travel and a serious poker affinity. Thanks for the money I took from you through numerous bluffs. Nick, you left a gap in the department and poker table since you took off to NYC. Thanks for the laughter and your dark humor, and the fact that you never kicked my ass for making you look like a fool in your PhD movie. 
And Rainer, you have always been a huge inspiration and example for me. Especially during our cooperation in the BrainMatters project I realized how your drive and enthusiasm can and does motivate so many people. Thank you.

None of my experiments would have worked without the great constructive comments and suggestions by our 'FFA'-group. Bernie, Francesco, Inge, Milene, Jao Joao, Marieke and recently expanded with Gojko: your expertise and insights have amazed me numerous times. Thanks for your honesty and support over the years! Also the students that have worked with me in this context, Mario Eduardo, Daan and especially Beatrice: your talents still inspire me. Thanks!

My data would have been nothing more than noise and artifacts without the beautiful brains of my subjects. Every statement in this thesis is based on your hemodynamics, following the patterns of my design. I couldn't begin to thank you for your time and dedication to my repetitive stimuli and tasks. Although all subjects contributed to the final results, I would like to thank one subject in particular, for he/she (trying to keep it anonymous here) simply has the best cortex-experiment overlap possible. I Think he or she knows.

Thank you, every other member of my department! Henk, Anke, Lars, Britta, Matteo, Marin, Aline, Alard, Amanda, Michael, Arie, Felix, Gesa, Valentin, Jan, Anna, Roberta, Martin, Bettina, Mehrdad, Kiki, Sanne, Mario and all those I may forget: it was my pleasure to share lunches, parties, discussions, conference trips and PhD movies with you guys. A special thanks to Rob, whose pieces of chocolate kept me going through the last days of writing this thesis. And last but certainly not least: Christl, Riny and Annemie - I can only begin to imagine what armageddon scenario's would have become reality without your implicit and explicit care and structure. And Heidi, as Bernie's secretary you were my portal into her agenda. Thanks for allowing me to see my supervisor.

Ik voel me verplicht om de rest van dit hoofdstuk in mijn moedertaal te schrijven. Jaap, verrekte nerd, aan jou heb ik natuurlijk helemaal niks gehad. Aan dat kansloze geklaag, gigabytes aan zeikmails en de uren tijdverspilling aan Peggle, poker en slechte films denk ik dan ook liever niet terug. Laat staan al die liters drank, de sigaren en het verzadigde vet dat ik bij jou voorgeschoteld kreeg. Bedankt man. Doede vanavond?

Mijn Maastrichtmatties. Jullie hebben alle aspecten van mijn $\mathrm{PhD}$ meegemaakt (mee moeten maken?). Van de vreugde over goede resultaten, artikelpublicaties en televisie-uitzendingen, tot de onvermijdelijke dippen die bij 
een onderzoeker horen. Jullie waren er altijd. Steef, Peet, Maarten, Anne, Sanne, Willie, Heleen, Yoeri, Loes, Tamara, bedankt voor de feesten, diners, scheldkanonnades bij het zombieknallen, de PPPPPPG\&G, de barbecues, de filmavonden, de weekendjes weg en de keren dat ik naar zuurstof happend op de grond lag te kronkelen als er een schoppende gorilla werd uitgebeeld. Bedankt voor jullie vriendschap al die jaren!

Mijn extra-Maastrichtmatties. Studeren in Maastricht en dan vervolgens ook nog een $\mathrm{PhD}$ doen in het verre zuiden. Vaak heb ik me beklaagd over het feit dat ik jullie te weinig zag, maar ik ben zo blij dat het weinige zien nooit een issue is geweest voor een goede vriendschap. Zien we elkaar een half jaar niet? Dan pikken we de onzinnige gesprekken op alsof er een dag tussen zat. Danny, Plien, Maarten, Maerten, Sander, Paul, Gijs, Hèn, verrekt'n Bèr, Bolle: wat zou mijn leven saai zijn geweest zonder de reizen naar Scandinavië, Frankrijk, Italië en Center Parcs, de 9 Brabantse Dagen, het gefilosofeer over wetenschap en politiek, het knaksen en de smerige foto's. Als weinig elkaar zien de bodem is voor al die vriendschap, hoop ik jullie verdomd weinig te blijven zien.

Oop en Oom. Ik heb altijd het gevoel gehad dat ik terecht ben gekomen waar ik nu ben mede door jullie. Oop, jouw creativiteit, handigheid en kiene puzzelgeest hebben me zoveel dingen geleerd over hoe ik problemen kan aanpakken. Oom, jouw inspirerende kijk op het leven, je grote interesse en je oog voor dingen die ik niet zie hebben me altijd laten nadenken hoe dingen in een grotere context thuishoren. Heel veel dank!

Mijn lieve broer en zussen. Voor jullie is het vaak een groot raadsel geweest hoe op mijn werk iedereen nog steeds gelooft dat ik een wetenschapper ben, terwijl ik thuis in Heeze nooit verder kom dan het niveau van een puber met een grijns op zijn smoel waaraan je kunt zien dat hij weer een streek heeft uitgehaald. Daan, Mijke, Imke, bedankt voor jullie interesse in wat ik doe, bedankt voor alle fijne thuismomenten en vooral bedankt voor wie jullie zijn. Soms als ik jullie na lange tijd weer zie, verbaast het me weer hoe volwassen jullie zijn. En dat zorgt er voor dat ik ongelofelijk trots ben om jullie grote broer te zijn.

$\mathrm{Pa}$ en Ma. Dit boekje vertegenwoordigt niet alleen 4 jaar onderzoek, maar staat vooral voor ruim 28 jaar zijn wie ik ben. Al de keuzes die ik maakte heb ik alleen kunnen maken omdat jullie er altijd achter stonden en er voor me waren als ik dat nodig had. Ik kan niet in woorden uitdrukken hoe dankbaar ik daar voor ben. Het meest trots ben ik op de momenten waar jullie trots op mij waren. Stiekem draag ik dit boekje dan ook aan jullie op. 
Yoanne, elke pagina in dit boek staat gelijk aan een moment dat ik het te druk had, moe was, of tegen je stond te klagen dat ik de hoop in m'n onderzoek verloren had. Met je eindeloze geduld, je vertrouwen en je positieve instelling heb je me eindeloos vaak weer opgebeurd en op weg geholpen, meer dan je zelf denkt. Ik kan makkelijk zeggen dat de inhoud van mijn $\mathrm{PhD}$ heel anders was verlopen zonder jou als thuisbasis. Ook al realiseerde ik me dat vooral vaak op de momenten dat je er even niet was :-). Bedankt voor wie je bent, pip. En blijf vooral jezelf. Fijn... zeu. 



\section{Curriculum Vitae}

Job van den Hurk was born on January $11^{\text {th }}, 1985$ in Heeze, The Netherlands. In 2004, he graduated from the Strabrecht College high school in Geldrop, and subsequently enrolled at Maastricht University at the Faculty of Psychology and Neuroscience. After obtaining his Bachelor of Science degree in 2007, he followed the Master's program in Cognitive Neuroscience. In his internship under supervision of Professor Elia Formisano, he studied the neural processing of spatial sounds using fMRI. After his graduation in 2008, Job continued to work in the field of Cognitive Neuroscience as a Ph.D. candidate under supervision of Professor Bernadette Jansma at the department of Cognitive Neuroscience and Neuroimaging at Maastricht University. Here he currently works as a postdoctoral research fellow. Next to conducting research, Job is also dedicated to the translation of his field of science to the public. He promotes neuroscience and psychology through lectures, science café's and the project BrainMatters (www.brainmatters.nl) that he founded.

In his spare time, Job enjoys to play guitar, meet friends and play games. 